Aus der Abteilung Ethik und Geschichte der Medizin

(Prof. Dr. med. C. Wiesemann)

im Zentrum Psychosoziale Medizin

der Medizinischen Fakultät der Universität Göttingen

\title{
Gruppennützige Forschung an Kindern und Jugendlichen
}

Ihre ethische und rechtliche Zulässigkeit unter besonderer Berücksichtigung der Bewertung von Vorsitzenden deutscher Ethikkommissionen

\author{
INAUGURAL-DISSERTATION \\ zur Erlangung des Doktorgrades \\ der Medizinischen Fakultät \\ der Georg-August-Universität zu Göttingen \\ vorgelegt von \\ Katrin Elisabeth Radenbach \\ aus \\ Lübeck
}

Göttingen 2006 
Dekan: Prof. Dr. med. C. Frömmel

1. Berichterstatterin: Prof. Dr. med. C. Wiesemann

2. Berichterstatter/-in:

3. Berichterstatter/-in:

Tag der mündlichen Prüfung: 


\section{Inhaltsverzeichnis}

Tabellenverzeichnis $\quad 5$

$\begin{array}{ll}\text { Abbildungsverzeichnis } & 6\end{array}$

$\begin{array}{ll}\text { Abkürzungsverzeichnis } & 8\end{array}$

1 Einleitung und Überblick 9

2 Grundlagen 10

2.1 Forschung am Menschen . . . . . . . . . . . . . . . . . . . 10

2.1.1 Notwendigkeit der Forschung an Kindern und Jugendlichen . . . 12

2.1.2 Aktuelle Situation in der pädiatrischen Pharmakotherapie . . . . . 13

2.1.3 Problematik der aktuellen Situation . . . . . . . . . . . . . . 15

2.2 Rechtliche und standesethische Rahmenbedingungen . . . . . . . . . . 16

2.2.1 Internationale Dokumente . . . . . . . . . . . . . 16

2.2.2 Nationale Dokumente . . . . . . . . . . . . . . . . . 19

2.2.3 Zusammenfassung .................. 23

2.3 Charakterisierung medizinethischer Begriffe . . . . . . . . . . . . . 24

2.3 .1 Nutzen . . . . . . . . . . . . . . . . . . . . . 24

2.3.2 Risiko und Belastung . . . . . . . . . . . . . . . 26

2.3.3 Beteiligung des Minderjährigen an Entscheidungen über seine

Versuchsteilnahme ................... 30

2.4 Ethikkommissionen . . . . . . . . . . . . . . . . . 35

2.4 .1 Geschichte ....................... 35

2.4.2 Aufgaben, Zusammensetzung und Arbeitsweise . . . . . . . . . . 36

3 Fragestellung der Arbeit 38

3.1 Risiko und Belastung . . . . . . . . . . . . . . . . . . . . 38

3.2 Beteiligung des Minderjährigen an Entscheidungen über seine Versuchs-

teilnahme ....................... . . 40

3.3 Ansatz zur Bearbeitung der Fragen . . . . . . . . . . . . . . . . . . . . . . 44

3.4 Kategorisierung . . . . . . . . . . . . . . . . . . 44

3.4 Nutzen . . . . . . . . . . . . . . . . . . . 44

3.4.2 Risiko und Belastung . . . . . . . . . . . . . . . . . . 44 
4 Auswertung von Forschungsanträgen $\quad 47$

4.1 Methodik . . . . . . . . . . . . . . . . . . . 47

4.1.1 Erhebung der Daten . . . . . . . . . . . . . . . . . . . . . . . . . . 47

4.2 Ergebnisse . . . . . . . . . . . . . . . . . . . 49

4.2.1 Nutzen der Studienprojekte . . . . . . . . . . . . . . . . . . 49

4.2.2 Risiko und Belastung der gruppennützigen Studienprojekte . . . . 50

4.2.3 Studien mit Arzneimitteln oder Medizinprodukten . . . . . . . . 51

4.2.4 Aufwandsentschädigung ....................... 51

4.2.5 Zusammenfassung .................. 51

5 Vignettenstudie $\quad 54$

5.1 Methodik ........................... 54

5.1 .1 Vignettenstudien . . . . . . . . . . . . . . 54

5.1 .2 Konzeption des Fragebogens . . . . . . . . . . . . . . 55

5.1 .3 Auswertung .................... 65

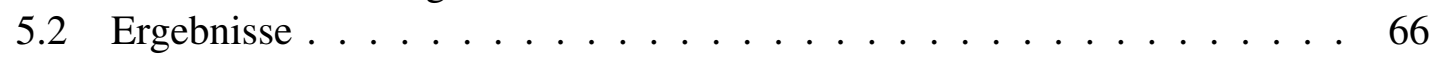

5.2.1 Charakteristika der Vorsitzenden . . . . . . . . . . . . 66

5.2.2 Zulässigkeit unterschiedlicher Studientypen . . . . . . . . . . . 66

5.2.3 Bedeutung der Beteiligung des Minderjährigen an Entscheidungen über seine Versuchsteilnahme . . . . . . . . . . . . 87

6 Diskussion 100

6.1 Forschung an Kindern und Jugendlichen . . . . . . . . . . . . . . . . . . . . . . . . . . . . . . .

6.2 Risiko und Belastung . . . . . . . . . . . . . . . . . . . . 101

6.3 Beteiligung des Minderjährigen an Entscheidungen über seine Versuchsteilnahme . . . . . . . . . . . . . . . 105

6.4 Ausblick . . . . . . . . . . . . . . . . . . . 108

$\begin{array}{lll}7 & \text { Zusammenfassung } & 109\end{array}$

8 Anhang $\quad \mathbf{1 1 0}$

8.1 Geplante Eingriffe gruppennütziger Forschungsprojekte . . . . . . . . . . 110

8.2 Originalfragebogen ............................ 111

9 Literaturverzeichnis $\quad 119$ 


\section{Tabellenverzeichnis}

3.1 Aussagen von Gesetzen und Empfehlungen zum zulässigen Risiko und zur zulässigen Belastung bei Forschung ohne direkten Eigennutzen . . . . 39

3.2 Aussagen von Gesetzen und Empfehlungen zur Beteiligung des Minderjährigen an Entscheidungen über seine Versuchsteilnahme . . . . . . . . 42

3.3 Aussagen von Gesetzen und Empfehlungen zur Begrenzung von Anreizen 43

3.4 Kategorisierung von Risiko und Belastung . . . . . . . . . . . . . . . . . 46

5.1 Übersicht der Charakteristika der Vignetten . . . . . . . . . . . . . . . . 64

5.2 Charakteristika der Vorsitzenden . . . . . . . . . . . . . . . . 67

5.3 Vignette 1: Kategorisierung der Anmerkungen und ihre Häufigkeit, bezogen auf die gewählte Antwortmöglichkeit . . . . . . . . . . . . . . . 69

5.4 Vignette 2: Kategorisierung der Anmerkungen und ihre Häufigkeit, bezogen auf die gewählte Antwortmöglichkeit . . . . . . . . . . . . . 71

5.5 Vignette 3: Kategorisierung der Anmerkungen und ihre Häufigkeit, bezogen auf die gewählte Antwortmöglichkeit . . . . . . . . . . . . . . . 74

5.6 Vignette 4: Kategorisierung der Anmerkungen und ihre Häufigkeit, bezogen auf die gewählte Antwortmöglichkeit . . . . . . . . . . . . . 77

5.7 Vignette 5: Kategorisierung der Anmerkungen und ihre Häufigkeit, bezogen auf die gewählte Antwortmöglichkeit . . . . . . . . . . . . . . . . 79

5.8 Vignette 6: Kategorisierung der Anmerkungen und ihre Häufigkeit, bezogen auf die gewählte Antwortmöglichkeit . . . . . . . . . . . 82

8.1 Geplante Eingriffe der gruppennützigen Forschungsprojekte (Anträge an die Ethikkommission der Universität Göttingen 1999-2003, N=57) . . . . 110 


\section{Abbildungsverzeichnis}

4.1 Anzahl der Anträge zur Forschung an Kindern und Jugendlichen an die Ethikkommission Göttingen 1999-2003 . . . . . . . . . . . . . . . . . 48

4.2 Anträge zur Forschung an Kindern und Jugendlichen an die Ethikkommission der Universität Göttingen 1999-2003: Nutzen in Kategorien . . . 50

4.3 Anträge zu gruppennütziger Forschung an Kindern und Jugendlichen an die Ethikkommission der Universität Göttingen 1999-2003: Risiko bzw. Belastung in Kategorien . . . . . . . . . . . . . . . . 51

4.4 Anträge zur Forschung an Kindern und Jugendlichen an die Ethikkommission der Universität Göttingen 1999-2003: Handelt es sich um Studien mit Arzneimitteln oder Medizinprodukten? . . . . . . . . . . . . . 52

4.5 Anträge zur Forschung an Kindern und Jugendlichen an die Ethikkommission der Universität Göttingen 1999-2003: Ist eine Aufwandsentschädigung für die Versuchspersonen vorgesehen? . . . . . . . . . . . . 52

5.1 Vignette 1, Frage 1: „Halten Sie eine solche Studie für vertretbar und würden Sie ein positives Votum einer Ethikkommission befürworten?“ . . 68

5.2 Vignette 2, Frage 1: „Halten Sie eine solche Studie für vertretbar und würden Sie ein positives Votum einer Ethikkommission befürworten?“ . . 70

5.3 Vignette 3, Frage 1: „Halten Sie eine solche Studie für vertretbar und würden Sie ein positives Votum einer Ethikkommission befürworten?““ . . 73

5.4 Vignette 4, Frage 1: „Halten Sie eine solche Studie für vertretbar und würden Sie ein positives Votum einer Ethikkommission befürworten?“ . . 76

5.5 Vignette 5, Frage 1: „Halten Sie eine solche Studie für vertretbar und würden Sie ein positives Votum einer Ethikkommission befürworten?“ . . 78

5.6 Vignette 6, Frage 1: „Halten Sie eine solche Studie für vertretbar und würden Sie ein positives Votum einer Ethikkommission befürworten?“ . . 81

5.7 Übersicht Frage 1: „Halten Sie eine solche Studie für vertretbar und würden Sie ein positives Votum einer Ethikkommission befürworten?" . . . . 84

5.8 Vignette 1, Fragen 2, 3 und 4: (Vorsitzende, die die Studie befürworteten) 88

5.9 Vignette 2, Fragen 2, 3 und 4: (Vorsitzende, die die Studie befürworteten) 89

5.10 Vignette 3, Fragen 2, 3 und 4: (Vorsitzende, die die Studie befürworteten) 91

5.11 Vignette 4, Frage 4: (Vorsitzende, die die Studie befürworteten) . . . . . . 92

5.12 Vignette 5, Fragen 2, 3 und 4: (Vorsitzende, die die Studie befürworteten) 93

5.13 Vignette 6, Fragen 2, 3 und 4: (Vorsitzende, die die Studie befürworteten) 94 
5.14 Übersicht Frage 2: „Halten Sie eine individuelle Aufklärung und Zustimmung des Kindes für erforderlich?" (Vorsitzende, die die Studie befürworteten) . . . . . . . . . . . . . . . . . 95

5.15 Übersicht Frage 3: „Wäre für Sie ein eindeutig abwehrendes Verhalten des Kindes ein Ausschluss- bzw. Abbruchkriterium?" (Vorsitzende, die die Studie befürworteten) . . . . . . . . . . . . . . . . . . 96

5.16 Übersicht Frage 4: „Halten Sie eine Aufwandsentschädigung für das Kind (Vignette 4: für die Eltern) für notwendig?" (Vorsitzende, die die Studie befürworteten) . . . . . . . . . . . . . . . . . . . . 99 


\title{
Abkürzungsverzeichnis
}

\author{
ALL ............ Akute lymphatische Leukämie \\ AMG \\ Arzneimittelgesetz \\ CFR \\ Code of Federal Regulations \\ CIOMS \\ Council for International Organizations of Medical Sciences \\ DHHS \\ Department of Health and Human Services \\ EEG \\ Elektroenzephalogramm \\ HKS \\ . Hyperkinetische Störung \\ LRS \\ Lese-Rechtschreib-Schwäche \\ MECP2 \\ Methyl CpG binding protein 2 \\ MPG \\ Medizinproduktegesetz \\ ZEKO \\ Zentrale Kommission zur Wahrung ethischer Grundsätze in der \\ Medizin und ihren Grenzgebieten (Zentrale Ethikkommission)
}




\section{Einleitung und Überblick}

Nationale und internationale Empfehlungen und Gesetze machen die Zulässigkeit von Forschung an Kindern und Jugendlichen davon abhängig, ob das Versuchsprojekt für den teilnehmenden Minderjährigen einen potentiellen direkten Eigennutzen beinhaltet. Wenn dies nicht der Fall ist, sein Ergebnis aber in Zukunft potentiell der Gruppe gleichaltriger oder gleichartig erkrankter Kinder nutzt, wird die Zulässigkeit einer Untersuchung an ihren Grad von Risiko und Belastung geknüpft.

Dabei wird in der Regel das so genannte minimale Risiko und die so genannte minimale Belastung als ein nicht zu überschreitendes Maß für die Zulässigkeit von Versuchen an Kindern und Jugendlichen ohne potentiellen direkten Eigennutzen festgelegt. Als weitere Voraussetzung für die Studienteilnahme eines Minderjährigen gilt, wenn auch in unterschiedlichem Ausmaß, dessen positive Einstellung zu seiner Rolle als Versuchsperson.

Im konkreten Fall entscheiden Ethikkommissionen darüber, ob geplante Forschungsprojekte an Kindern und Jugendlichen durchgeführt werden dürfen. In welchem Maß sie sich bei diesen Entscheidungen zur Forschung an Kindern und Jugendlichen ohne potentiellen direkten Eigennutzen am Grad von Risiko und Belastung orientieren, soll Gegenstand dieser Arbeit sein. Weiterhin soll erfasst werden, welche Bedeutung Vorsitzende von Ethikkommissionen der Beteiligung eines Minderjährigen an Entscheidungen über seine Versuchsteilnahme beimessen. Vor dem Hintergrund dieser Ergebnisse wird diskutiert werden, inwieweit aktuelle Empfehlungen und Gesetze die Bedingungen für die Zulässigkeit von Forschung an Kindern und Jugendlichen ohne potentiellen direkten Eigennutzen präzise genug regeln.

Die Arbeit ist folgendermaßen aufgebaut: Der erste Teil beschreibt, warum Forschung an Kindern und Jugendlichen eine besondere Betrachtung verdient. Die wichtigsten nationalen und internationalen rechtlichen und standesethischen Rahmenbedingungen der Forschung an Kindern und Jugendlichen werden dargestellt, die ethischen Begriffe, auf denen sie beruhen, charakterisiert und das Verfahren zur Begutachtung von Forschungsprojekten durch Ethikkommissionen beschrieben. Einer Präzisierung der verwendeten Begriffe und der Formulierung der Fragestellung folgt die Darstellung zweier für diese Arbeit durchgeführter empirischer Untersuchungen. Die Ergebnisse dieser Untersuchungen werden vor dem Hintergrund aktueller Gesetze und Empfehlungen diskutiert. Zuletzt werden Schlussfolgerungen gezogen, und es wird ein Ausblick auf mögliche weiterführende Forschungen gegeben. 


\section{Grundlagen}

\subsection{Forschung am Menschen}

Die naturwissenschaftlich fundierten Methoden der Diagnostik und Therapie von Krankheiten beruhen auf Ergebnissen von Forschung, die nach bestimmten Regeln abläuft. Ein großer Bereich der Forschung am Menschen ist die Arzneimittelforschung. Die Entwicklung neuer Arzneimittel bis zur Zulassung ${ }^{1}$ ist ein komplexer, Jahre dauernder Prozess, der mit sehr hohen Kosten verbunden ist. Er beginnt in vitro im Labor mit der Molekülfindung und Entwicklung einer Testsubstanz. Anschließend werden Tiere in die präklinische Prüfung einbezogen. ${ }^{2}$ Das neue Medikament wird auf Sicherheit, Kinetik, Toxizität, $\mathrm{Mu}-$ tagenität, Teratogenität und Kanzerogenität getestet.

Der therapeutische Nutzen eines Arzneimittels und das mit ihm verbundene Risiko können nur nach Anwendung am Menschen richtig eingeschätzt werden, deshalb folgt dem Tierversuch die klinische Prüfung. Sie besteht in Deutschland aus drei Phasen und einer vierten Phase nach Zulassung des neuen Medikaments ${ }^{3}$. Im Anschluss daran wird entschieden, ob das Medikament eine Zulassungsverlängerung erhält und ob es weiterhin

\footnotetext{
${ }^{1}$ Das Zulassungsverfahren für neue Arzneimittel ist erst seit 1961 vorgeschrieben. Zuvor wurden neue Medikamente lediglich von den Bundesländern registriert.

${ }^{2}$ Zur Problematik von Tierversuchen in der medizinische Forschung vgl. Blumer 1999, Klein 1998

${ }^{3}$ In Phase 1 wird die Substanz in einer offenen Studie ohne Kontrollgruppe an einer geringen Zahl gesunder Probanden getestet. Es geht um Verträglichkeit und Pharmakokinetik des neuen Arzneimittels. Ferner wird evaluiert, bei welcher Dosis das Medikament wirksam ist und bei welcher Dosis Nebenwirkungen auftreten. In Phase 2 werden circa 100-300 Patienten in die Prüfung des Medikaments einbezogen. In dieser Phase geht es um die Ermittlung der optimalen Arzneimitteldosis im Rahmen einer klinischen, kontrollierten, randomisierten und doppelblinden Studie. Außerdem wird wiederum die Verträglichkeit beobachtet, und es werden Wechselwirkungen registriert. Anschließend nehmen etwa 1000 Patienten an oft multizentrisch organisierten Phase-3-Studien teil. Mit ein oder zwei Dosen des in Phase 2 ermittelten Dosisbereichs wird im Rahmen eines kontrollierten klinischen Versuchs (doppelblind, randomisiert) ein statistisch gesicherter Beweis der therapeutisch gewünschten Wirkung im Vergleich zur Standardtherapie geführt. Die Verträglichkeit des Medikaments wird weiterhin beobachtet. An Phase 3 schließt sich die Entscheidung über die Zulassung des neuen Medikaments durch das Bundesinstitut für Arzneimittel und Medizinprodukte an. Für die Zulassung von Impfstoffen und Sera ist das Paul-Ehrlich-Institut zuständig. Das Medikament erhält einen Eigennamen und kann von Ärzten verordnet werden. Während der nächsten 5 Jahre wird seine Anwendung im Rahmen der Phase 4 der klinischen Prüfung beobachtet. Häufig werden seltene Nebenwirkungen erst während dieser Phase offenkundig (Gundert-Remy 2001).
} 
der Verschreibungspflicht eines Arztes unterliegen soll oder rezeptfrei verkauft werden kann.

Der kontrollierte klinische Versuch ist das etablierte Verfahren zur Feststellung erwünschter und unerwünschter Wirkungen eines Arzneimittels (Gundert-Remy 2001). Er ist charakterisiert durch die randomisierte Zuteilung der Versuchspersonen zu einer Verumgruppe (deren Mitglieder mit dem zu prüfenden Medikament behandelt werden) und einer Kontrollgruppe (deren Mitglieder ein etabliertes Medikament oder ein Placebopräparat erhalten). Studien können als offene Studien, als Einfach- oder als Doppelblindstudien durchgeführt werden. Am aussagekräftigsten sind Doppelblindstudien, bei denen weder Arzt noch Patient wissen, welcher Therapiegruppe der Patient angehört.

Vor Beginn jedes klinischen Versuches muss ein Prüfplan erstellt werden, der die Grundlage für die Begutachtung der Studie durch die Ethikkommission liefert. ${ }^{4}$

Eine weitere Art von Forschung am Menschen ist die Erhebung von Normal- oder Referenzwerten des menschlichen Körpers und seiner Funktionen. Für die Diagnostik von Normabweichungen und Krankheiten ist es unverzichtbar, sich auf solche Standardwerte zu berufen, die an gesunden Menschen erhoben wurden. Dies gilt beispielsweise für Referenzwerte der Körpergröße in Abhängigkeit des Alters, des arteriellen Blutdrucks oder für Laborparameter, die laboranalytisch Aufschluss über den Zustand menschlicher Organe geben können. Weiterhin gibt es epidemiologische Studien, Krankheitsursachenforschung und viele andere mehr.

Arzneimittelstudien werden in der Regel an Männern und älteren Frauen durchgeführt. Risikogruppen bei der klinischen Prüfung von Arzneimitteln sind z. B. Schwangere, Frauen im gebärfähigen Alter und Angehörige so genannter vulnerabler Gruppen.

Unter vulnerablen Gruppen versteht man bezüglich der Forschung am Menschen Personengruppen, deren Einwilligungsfähigkeit eingeschränkt ist oder die in ihrer Entscheidung für oder gegen eine Studienteilnahme nicht frei sind. Dazu gehören beispielsweise Minderjährige, und außerdem demente, geistig behinderte und in Einrichtungen untergebrachte Personen, sowie Menschen, die sich in Abhängigkeitsverhältnissen zu Studienverantwortlichen befinden. Angehörige dieser Gruppen werden in der Regel nicht in Arzneimittelstudien eingeschlossen. Diese Tatsache führt allerdings zum Beispiel in Bezug auf Schwangere ${ }^{5}$ und Minderjährige dazu, dass viele Medikamente nicht für diese Personengruppen zugelassen sind (siehe Abschnitt 2.1.2).

\footnotetext{
${ }^{4}$ Ein Prüfplan soll Angaben enthalten über den theoretischen Hintergrund der Studie, die Hauptzielgröße zur Bestimmung von Wirkungen und Nebenwirkungen und über weitere Prüfparameter, die Begründung der Kontrollbehandlung, die Auswahlkriterien für Probanden oder Patienten und die voraussichtlich notwendige Anzahl, über die schriftlichen Aufklärungs- und Einwilligungsformulare, den praktischen Ablauf der klinischen Prüfung und das geplante Vorgehen zur Auswertung der Ergebnisse (GundertRemy 2001).

${ }^{5}$ Die Problematik der Forschung an Schwangeren ist ein aktuelles Forschungsthema (vgl. Biller-Andorno und Wild 2003).
} 
Weiterhin wird vermutet, dass ein Grund für fehlende klinische Studien in der Kinderheilkunde und Jugendmedizin und in der Kinder- und Jugendpsychiatrie bei der pharmazeutischen Industrie zu suchen ist, für die es sich langfristig deutlich rentabler und weniger aufwändig darstellt, Arzneimittelstudien an Erwachsenen durchzuführen und Medikamente für Erwachsene zu entwickeln (Brochhausen 2005).

So sind Kinder und Jugendliche therapeutic orphans (Shirkey 1968), für die wenige geprüfte Medikamente vorliegen, die auf ihre Bedürfnisse und Krankheiten zugeschnitten sind.

\subsubsection{Notwendigkeit der Forschung an Kindern und Jugendlichen}

Warum muss spezifisch an Kindern und Jugendlichen geforscht werden? Lässt sich die medizinisch notwendige Forschung nicht an Erwachsenen realisieren?

Je jünger Kinder sind, umso mehr muss mit entwicklungsspezifischen Abweichungen der Pharmakokinetik und der Wirksamkeit der Medikamente von einem Erwachsenen im mittleren Lebensalter gerechnet werden, und umso dringender erforderlich sind entwicklungsspezifische Informationen zum Dosierungsschema (Walter-Sack und Haefeli 2001). Kindliche Organe unterscheiden sich nicht nur quantitativ von ausgewachsenen Organen. Auch auf der Stoffwechselebene gibt es große Abweichungen: Neugeborene scheiden Arzneistoffe beispielsweise langsamer aus, weil Leber und Nieren noch nicht ausgereift sind. Kleinkinder und Kinder bis acht Jahre dagegen metabolisieren Arzneimittel schneller als Erwachsene. Um sie optimal zu behandeln, müssen Medikamente daher relativ hoch dosiert werden (Rosenthal und Seyberth 2001). Es genügt nicht, die Dosis des Wirkstoffs dem Gewicht eines Kindes anzupassen.

Ergebnisse aus der Forschung an Erwachsenen können nicht einfach auf die Therapie von Minderjährigen übertragen werden. Medikamente können bei Kindern und Jugendlichen eine andere Wirkung und andere unerwünschte Arzneimittelwirkungen als bei Erwachsenen zeigen: Beispielsweise ruft Metoclopramid, ein Dopaminantagonist, der bei Erwachsenen ohne gravierende Nebenwirkungen zur Bekämpfung von gastrointestinalen Beschwerden wie Übelkeit und Erbrechen eingesetzt werden kann, bei jüngeren Kindern ein dyskinetisches Syndrom hervor (Heintze 2000). Um eine angemessene Behandlung zu ermöglichen, müssen daher pharmakokinetische und pharmakodynamische Vorgänge bei Kindern und Jugendlichen gesondert erforscht werden.

Ein weiteres Argument für die Notwendigkeit speziell pädiatrischer Forschung ist die Tatsache, dass sich viele behandlungsbedürftige Krankheiten bereits im frühen Kindesalter 
manifestieren, wie z. B. der Diabetes mellitus Typ I, oder anders verlaufen als bei Erwachsenen, wie z. B. Infektionen mit dem Herpes-simplex-Virus Typ $1^{6}$.

Außerdem spielt in der Pädiatrie die Beobachtung einer altersentsprechenden körperlichen und geistigen Entwicklung eine wichtige Rolle. Dazu gehört die Erhebung von Normal- und Referenzwerten, die nur durch die Untersuchung vieler Heranwachsender geschehen kann. Auch die Prävention von Krankheiten, insbesondere von Infektionskrankheiten, ist speziell im Kindesalter von großer Bedeutung. In diesem Zusammenhang ist die Entwicklung und die Erprobung von Impfstoffen wichtig.

\subsubsection{Aktuelle Situation in der pädiatrischen Pharmakotherapie}

Es ist Ärzten möglich, im Rahmen des so genannten unlicensed use Medikamente zu verordnen, die im entsprechenden Land (noch) nicht zugelassen sind und aus dem Ausland bezogen werden. Außerdem können Medikamente auch außerhalb ihres empfohlenen Zulassungsbereichs, also off-label verschrieben werden. Beispielsweise therapiert ein Arzt off-label, wenn er Medikamente für andere als für die zugelassenen Indikationen einsetzt oder wenn er sich nicht an die Dosierungsangaben bzw. an den vorgegebenen Altersbereich hält (Conroy et al. 2000). Ärzte nutzen die Möglichkeit, Medikamente ohne Zulassung oder außerhalb ihres Zulassungsbereichs zu verordnen, wenn sie meinen, eine optimale Therapie nicht im Rahmen zugelassener Medikamente und ihres vorgegebenen Einsatzbereichs vornehmen zu können.

In der Pädiatrie sind solche Verordnungen häufig: Eine Dokumentation aus Krankenhäusern in fünf europäischen Ländern (Großbritannien, Schweden, Niederlande, Italien und Deutschland) zeigte, dass zwei Drittel der Kinder und Jugendlichen (67\%) auf den beteiligten, meist allgemeinpädiatrischen Stationen unlicensed oder off-label verschriebene Medikamente erhielten. Fast die Hälfte aller Arzneimittel (46\%), die die Patienten im Laufe ihres Krankenhausaufenthaltes erhielten, waren unlicensed oder wurden offlabel gegeben. Beispielhaft nennen die Autoren der Studie die nur einmalige Gabe des Antibiotikums Tobramycin pro Tag, bei Zulassung des Medikaments ausschließlich für die zweimalige Gabe pro Tag, die Gabe von höheren als den empfohlenen Dosen des Kortikosteroids Fluticason oder die häufigere Frequenz der Verabreichung von Beta-2Sympathomimetika wie Salbutamol als in den Fachinformationen vorgegeben war. Ein Problem ist die weit verbreitete Anwendung inhalativer Kortikosteroide in der Therapie des kindlichen Asthma bronchiale: Es gibt wenig Daten über den wachstumssuppressiven

\footnotetext{
${ }^{6}$ Diese Infektion verläuft bei $90 \%$ der immunkompetenten Erwachsenen asymptomatisch, manifestiert sich aber bei Neugeborenen fast immer klinisch im Sinne einer Gingivostomatitis, die mit Fieber, Schluckbeschwerden und Nahrungsverweigerung ein schweres Krankheitsbild ergeben kann (Scholz 2002).
} 
Effekt von Kortikosteroiden bei Kindern unter zwei Jahren, und so können diese Medikamente in den von der Dokumentation erfassten Zentren nur off-label eingesetzt werden. Die am häufigsten off-label verschriebenen Medikamentengruppen waren Analgetika und Bronchodilatatoren. Die meisten noch nicht zugelassenen Medikamente wurden in einem Zentrum in Rotterdam in den Niederlanden verordnet, das den höchsten Anteil von Kindern und Jugendlichen mit schweren und komplexen Krankheitsbildern verzeichnete (Conroy et al. 2000).

Im ambulanten Bereich in Deutschland stellt sich die Situation anders dar: Eine Auswertung von 1,74 Millionen Rezepten aus dem Jahr 1999 für Minderjährige bis zu 16 Jahren zeigte, dass nur $13 \%$ der von niedergelassenen Pädiatern, Allgemeinmedizinern und Internisten verschriebenen Medikamente unlicensed waren oder off-label verschrieben wurden. Beispiele sind die Gabe von Xylometazolin bei Babys und Kleinkindern in höheren als den empfohlenen Dosen und die Therapie von Babys mit Salbutamol, dessen Wirksamkeit erst bei Kindern ab eineinhalb Jahren erwiesen ist (Bücheler et al. 2002). Einen etwas höheren Anteil von unlicensed use und off-label use im ambulanten Bereich zeigte eine Studie aus den Niederlanden, die 68.019 Rezepte aus dem Jahr 2000 auswertete: $17 \%$ der verschriebenen Medikamente waren nicht zugelassen und $23 \%$ waren außerhalb ihres Zulassungsbereichs verordnet worden (Schirm et al. 2002).

Abweichungen von der empfohlenen Anwendungsweise führen ebenfalls dazu, dass Verordnungen als off-label klassifiziert werden. Eine Studie aus Schottland analysierte die prozentualen Anteile dieser Verschreibungen: Es zeigte sich, dass 26,1\% der 167.865 Kinder und Jugendlichen, die bei einem von insgesamt 161 Allgemeinmedizinern registriert waren, im Laufe eines Jahres mindestens ein Medikament off-label erhielten. Die häufigste Ursache dafür war die Verordnung eines Medikaments in einer niedrigeren als der empfohlenen Dosis (40-50\%). Meist betraf dies Antihistaminika und Antibiotika. Ebenfalls häufig war die Verordnung einer höheren als der empfohlenen Dosis (35\%), oft von Medikamenten zur Therapie des Asthma bronchiale, von topischen Kortikosteroiden oder Laxanzien. Seltener waren Abweichungen von der Altersempfehlung (6-16\%) oder von der Art der Medikamentengabe (5-10\%) die Ursache für eine offlabel-Klassifizierung (Ekins-Daukes et al. 2004).

In der ambulanten Patientenversorgung werden offensichtlich weniger Medikamente ohne Zulassung oder off-label verschrieben als unter stationären Bedingungen. Da in dem von Conroy et al. erfassten Zentrum, in dem der höchste Anteil von Kindern und Jugendlichen mit schweren, komplexen Krankheitsbildern behandelt wurde, die meisten Medikamente ohne Zulassung verabreicht wurden (Conroy et al. 2000), lässt sich vermuten, dass besonders für Minderjährige mit schweren Krankheitsbildern, die einer stationären Therapie bedürfen, zu wenig Arzneimittelforschung betrieben wird. 


\subsubsection{Problematik der aktuellen Situation}

Ärzte sind gezwungen, bei Minderjährigen Medikamente außerhalb des zugelassenen Bereiches anzuwenden, da Kinder und Jugendliche mit den für sie zugelassenen Medikamenten nicht zufriedenstellend behandelt werden können. Damit sind Minderjährige in der medikamentösen Therapie Erwachsenen gegenüber deutlich benachteiligt. Sie werden nicht evidenzbasiert, sondern nach den fachlichen Vermutungen und persönlichen Erfahrungen der behandelnden Ärzte therapiert. Die Anwendung von nicht zugelassenen Medikamenten und der off-label use führen zu einer höheren Wahrscheinlichkeit des Auftretens ernster Nebenwirkungen: In einer achtmonatigen prospektiven Studie, die auf einer pädiatrischen Isolierstation der Universitätsklinik Erlangen-Nürnberg stattfand, zeigte sich unter Therapie mit unlicensed oder off-label angewandten Medikamenten ein signifikant höheres Risiko von Nebenwirkungen als bei Anwendung regulär zugelassener Medikamente (Neubert et al. 2004). Zu einem ähnlichen Ergebnis kamen schwedische und britische Wissenschaftler (Turner et al. 1999, Ufer et al. 2004).

Ein Arzt macht sich durch den Einsatz nicht zugelassener Medikamente oder durch Offlabel-Therapie nicht strafbar. Trotzdem begibt er sich damit auf eine medizinische und juristische Gratwanderung. Im Fall eines off-label use trägt er nicht nur das allgemeine Haftungsrisiko, sondern auch das Haftungsrisiko für unerwünschte Arzneimittelwirkungen (Wemhöner und Frehse 2004). Ebenso wurde aber 1990 Ärzten ein Behandlungsfehler angelastet, weil sie einem an viraler Meningitis erkrankten Kind die Behandlung mit Aciclovir zeitweise vorenthielten, weil das Arzneimittel noch nicht für diese Indikation zugelassen war (Wemhöner und Frehse 2004).

Für die minderjährigen Patienten und für ihre behandelnden Ärzte ist die Situation demnach schwierig und riskant. Der Pädiater Martin Hulpke-Wette charakterisierte sie 2005 mit dem Begriff der Arzneimittelunsicherheit. Immer wieder fordern Pädiater die häufigere Durchführung kontrollierter klinischer Studien auch bei Minderjährigen (Hulpke-Wette 2005, Seyberth 1999). Eine Verbesserung von Diagnose und Therapie bei Minderjährigen sei nur durch mehr klinische Forschung möglich. Unterlasse man sie, verzichte man bewusst auf eine optimale Patientenversorgung (Walter-Sack und Haefeli 2001).

Die Zulässigkeit von Forschung an Kindern und Jugendlichen wird in den für Deutschland relevanten Gesetzen und nationalen und internationalen Empfehlungen an bestimmte Bedingungen geknüpft. Im folgenden Abschnitt 2.2 werden diese Gesetze und Empfehlungen vorgestellt und ihre Bedingungen für Forschung an Kindern und Jugendlichen erläutert. 


\subsection{Rechtliche und standesethische Rahmenbedingungen}

Forschung an Menschen wird international und national durch verschiedene Dokumente mit teils empfehlendem und teils rechtsverbindlichem Charakter geregelt. Grundsätzlich verlangen alle Gesetze und Empfehlungen eine unabhängige Prüfung von Studienvorhaben an Menschen durch Ethikkommissionen. ${ }^{7}$ Für die Zulässigkeit von Forschung an Kindern und Jugendlichen bzw. an vulnerablen Gruppen, über die im konkreten Fall in Deutschland die Ethikkommissionen zur Forschung am Menschen zu entscheiden haben, stellen Gesetze und Empfehlungen jedoch unterschiedliche Bedingungen.

\subsubsection{Internationale Dokumente}

\subsubsection{Deklaration von Helsinki des Weltärztebundes}

Die Deklaration von Helsinki gilt als ,wichtigstes international anerkanntes Dokument ärztlicher Standesauffassung zur medizinischen Forschung am Menschen“ (Taupitz 2001, S. A 2413). Sie entstand 1964 als Weiterentwicklung des Nürnberger Kodex auf der 18. Generalversammlung des Weltärztebundes in Helsinki. Inzwischen wurde sie fünfmal revidiert, zuletzt im Jahr 2000 in Edinburgh mit jeweils einer Note of Clarification in den Jahren 2002 und 2004 (Deklaration von Helsinki 2004).

Grundsatz 24 bezieht sich ausdrücklich auf Forschung an Minderjährigen: "For a research subject who is legally incompetent, physically or mentally incapable of giving consent or is a legally incompetent minor, the investigator must obtain informed consent from the legally authorized representative in accordance with applicable law. These groups should not be included in research unless the research is necessary to promote the health of the population represented and this research cannot instead be performed on legally competent persons." Welcher Grad an Risiko und Belastung dabei vertretbar ist, wird nicht spezifisch für Minderjährige präzisiert.

Grundsatz 25 geht auf die Beteiligung Minderjähriger an Entscheidungen über ihre Versuchsteilnahme ein: "When a subject deemed legally incompetent, such as a minor child, is able to give assent to decisions about participation in research, the investigator must obtain that assent in addition to the consent of the legally authorized representative."

\footnotetext{
${ }^{7}$ Grundsatz 13 der Deklaration von Helsinki (Deklaration von Helsinki 2004), Guideline 2 und Guideline 3 des CIOMS (Guidelines des CIOMS 2002), Artikel 2 k), Artikel 3 (2) a), Artikel 6 und Artikel 10 a) der Richtlinie 2001/20/EG (Richtlinie 2001/20/EG 2001), Artikel 16 iii des Menschenrechtsübereinkommens zur Biomedizin des Europarates (Menschenrechtsübereinkommen des Europarates 1997), § 40 (1) und $\S 42$ (1) des aktuellen AMG (AMG 2004), § 20 (7) und $§ 20$ (8) des MPG (MPG 2002) und § 15 (1) der (Muster-)Berufsordnung ((Muster-)Berufsordnung 2004).
} 


\subsubsection{Richtlinien des CIOMS}

Die International Ethical Guidelines for Biomedical Research Involving Human Subjects des Council for International Organizations of Medical Sciences (CIOMS) in Zusammenarbeit mit der World Health Organization von 2002 nehmen ausführlich Stellung zur Forschung an Angehörigen vulnerabler Gruppen und speziell an Minderjährigen (Guidelines des CIOMS 2002).

Für Forschung an Kindern und Jugendlichen werden folgende Bedingungen formuliert: "Before undertaking research involving children, the investigator must ensure that:

- the research might not equally well be carried out with adults;

- the purpose of the research is to obtain knowledge relevant to the health needs of children;

- a parent or legal representative of each child has given permission;

- the agreement (assent) of each child has been obtained to the extent of the child's capabilities; and,

- a child's refusal to participate or continue in the research will be respected." (Guideline 14$)^{8}$

Welches Risiko einer kindlichen Versuchsperson zugemutet werden kann, wird nicht präzisiert. In Guideline 9 wird darauf eingegangen, dass Risiken, die mit Versuchen an ,individuals incapable of giving informed consent" einhergehen, die Risiken einer medizinischen oder psychologischen Routineuntersuchung nicht überschreiten dürften. Nur

\footnotetext{
${ }^{8}$ Die Vorgaben zur Beteiligung des Minderjährigen an Entscheidungen über seine Versuchsteilnahme werden im Kommentar zur Richtlinie präzisiert: Auch Minderjährige könnten Informed-consentProzeduren sinnvoll durchlaufen und anschließend ihrer Versuchsteilnahme zustimmen. Wenn es bei Kindern, die zu klein seien, um ihre Zustimmung zu formulieren, zu einer bewussten Ablehnung (deliberate objection) der Studienteilnahme bzw. einzelner damit verbundener Maßnahmen komme, die von unspezifischen Reaktionen auf Stimuli unterscheidbar sei, solle sie respektiert werden. Ausnahmen seien Fälle, in denen ein Minderjähriger unbedingt behandelt werden müsse und ein Medikament benötige, das nur im Rahmen klinischer Forschung verfügbar sei, wenn für ihn ein direkter Eigennutzen von der Forschung zu erwarten sei und keine Alternative zur Verfügung stehe. Bei älteren Kindern und Jugendlichen solle in einem solchen Fall eine Ethikkommission den Konflikt begutachten. Grundsätzlich solle Forschung vorzugsweise an älteren Kindern und Jugendlichen realisiert werden (Commentary on Guideline 14).

$\mathrm{Ab}$ einem Alter von 12 oder 13 Jahren sei ein informed consent des Kindes bzw. Jugendlichen möglich. Eine kindliche Zustimmung solle in jedem Alter gesucht werden. In seltenen Fällen sei bei bestimmten Studien nach Entscheidung einer Ethikkommission eine Forschungsteilnahme ohne Information und Einverständnis der Eltern möglich, nämlich bei schwangeren, verheirateten oder unabhängig von ihren Eltern lebenden Minderjährigen oder bei Studien zu brisanten Themen wie Sexualität, Missbrauch, Drogen oder häuslicher Gewalt. Eltern bzw. die gesetzlichen Vertreter und das Kind sollten während der Studie ärztlich und psychologisch betreut werden (Commentary on Guideline 14).
} 
in Ausnahmefällen könne durch Ethikkommissionen eine geringfügige Überschreitung (, slight or minor increase “) erlaubt werden (Guideline 9). ${ }^{9}$

Guideline 7 „Inducement to participate“ macht Vorgaben für den Fall, dass gesetzliche Vertreter Gewinn aus der Versuchsteilnahme ihres Schutzbefohlenen ziehen könnten, und legt fest: "A guardian asked to give permission on behalf of an incompetent person should be offered no recompense other than a refund of travel and related expenses."

\subsubsection{Richtlinie 2001/20/EG (Gute klinische Praxis) des europäischen Parlaments und des Rates}

Die Richtlinie 2001/20/EG des europäischen Parlaments und des Rates von 2001 ,zur Angleichung der Rechts- und Verwaltungsvorschriften der Mitgliedstaaten über die Anwendung der guten klinischen Praxis bei der Durchführung von klinischen Prüfungen mit Humanarzneimitteln" ist auf europäischer Ebene rechtswirksam und wurde auch in Deutschland im Jahr 2004 in nationales Recht umgesetzt (Richtlinie 2001/20/EG 2001). Artikel 4 geht auf Minderjährige als Prüfungsteilnehmer ein und nennt folgende Bedingungen für die Zulässigkeit von Studien: Forschung an Kindern und Jugendlichen müsse eine jederzeit ohne Nachteile widerrufbare Einwilligung der Eltern oder des gesetzlichen Vertreters vorausgehen, die dem mutmaßlichen Willen des Minderjährigen entspricht (Artikel 4 a). Minderjährige sollten von pädagogisch geschultem Personal aufgeklärt werden (Artikel 4 b). Die Ablehnung einer Studienteilnahme durch Minderjährige, die die Aufklärungsinformationen adäquat beurteilen können, solle berücksichtigt werden (Artikel 4c).

Anreize oder finanzielle Vergünstigungen für eine Prüfungsteilnahme sollten mit Ausnahme einer Entschädigung nicht geboten werden (Artikel $4 \mathrm{~d}$ ).

Forschung, die „für die Patientengruppe mit einem direkten Nutzen“ verbunden ist, gilt als zulässig (Artikel 4 e). Sie soll so geplant sein, ,,dass sie unter Berücksichtigung der Erkrankung und des Entwicklungsstadiums mit möglichst wenig Schmerzen, Beschwerden, Angst und anderen vorhersehbaren Risiken verbunden“" ist (Artikel 4 g). Risikoschwelle und Belastungsgrad müssten eigens definiert und ständig überprüft werden. Ethikkommissionen, die über Forschungsprojekte mit Kindern entscheiden, müssten über Kenntnisse auf dem Gebiet der Kinderheilkunde verfügen oder sich in diesem Bereich beraten lassen (Artikel 4 h).

\footnotetext{
${ }^{9}$ Beispiele dafür seien etwa „additional lumbar punctions or bone-marrow aspirations in children with conditions for which such examinations are regularly indicated in clinical practice" (Commentary on Guideline 9). Außerdem betont der Kommentar zur Richtlinie: "The requirement that the research interventions be reasonably commensurate with clinical interventions that subjects may have experienced or are likely to experience for the condition under investigation is intended to enable them to draw on personal experience as they decide whether to accept or reject additional procedures for research purposes."
} 


\subsubsection{Menschenrechtsübereinkommen zur Biomedizin des Europarates}

Das „Menschenrechtsübereinkommen zur Biomedizin des Europarates“ befasst sich nicht spezifisch mit Forschung an Minderjährigen. Artikel 6 regelt den Schutz einwilligungsunfähiger Personen bei Maßnahmen, die die Gesundheit betreffen, und Artikel 16 und 17 regeln den Schutz einwilligungsunfähiger Personen bei Forschungsvorhaben (Menschenrechtsübereinkommen des Europarates 1997). Das Menschenrechtsübereinkommen stieß gerade wegen seiner Regelung der Forschung an einwilligungsunfähigen Personen in Teilen der deutschen Öffentlichkeit auf geringe Akzeptanz. Bisher wurde es von Deutschland noch nicht ratifiziert, soll aber wegen seiner Bedeutung in der ethischen Diskussion über Forschung am Menschen hier trotzdem aufgeführt werden.

Forschung an einwilligungsunfähigen Personen wird erlaubt, wenn sie zum Ziel hat, „durch eine wesentliche Erweiterung des wissenschaftlichen Verständnisses des Zustands, der Krankheit oder der Störung der Person letztlich zu Ergebnissen beizutragen, die der betroffenen Person selbst oder anderen Personen nützen können, welche derselben Altersgruppe angehören oder an derselben Krankheit oder Störung leiden oder sich in demselben Zustand befinden,“ und wenn die Forschung „für den Betroffen nur mit einem minimalen Risiko und mit einer minimalen Belastung" einhergehe (Artikel 17, (2) i, ii).

Das Menschenrechtsübereinkommen gibt vor, dass bei Interventionen an minderjährigen Personen die Einwilligung eines gesetzlichen Vertreters vorliegen muss (Artikel 6 (2)). Die Ansicht der minderjährigen Person ,,wird als ein mit zunehmendem Alter und entsprechender Reife an Bedeutung gewinnender Faktor berücksichtigt“" (Artikel 6(2)).

Voraussetzung für die Forschung an einwilligungsunfähigen Personen sei, dass sie nicht an einwilligungsfähigen Versuchspersonen durchgeführt werden könne, die Einwilligung des gesetzlichen Vertreters und keine ablehnende Haltung der Versuchsperson vorliege (Artikel 16i.-v., Artikel 17 (1) i.-v.).

\subsubsection{Nationale Dokumente}

\subsubsection{Gesetz über den Verkehr mit Arzneimitteln (Arzneimittelgesetz)}

Das deutsche Arzneimittelgesetz (AMG) wurde 2004 novelliert. In der früheren Fassung von 1994 wurde bezüglich der Forschung an Minderjährigen nach Diagnostika und Prophylaktika (\$ 40(4)) auf der einen und Therapeutika (§41) auf der anderen Seite differenziert, was als Ursache für Interpretationsschwierigkeiten kritisiert wurde (Dahl und Wiesemann 2001). Die Frage nach dem zumutbaren Risiko wurde in der alten Fassung des AMG nicht berücksichtigt. Aufgrund divergierender juristischer Interpretationen (Fröhlich 1999, Taupitz 1999, Wachenhausen 2000) war nicht eindeutig, ob Arzneimittel- und 
Medizinprodukteforschung ohne direkten potentiellen Nutzen für minderjährige Versuchsteilnehmer zulässig war oder nicht (Wiesemann und Dahl 2003). Gefordert wurde in der alten Version, dass die klinische Prüfung an Erwachsenen ,keine ausreichenden Prüfergebnisse erwarten lassen“ dürfe (AMG 1994, § 40 (4) 3.) und dass außerdem die Einwilligung des gesetzlichen Vertreters sowie die schriftliche Einwilligung des Minderjährigen vorliege, wenn er in der Lage sei, „Wesen, Bedeutung und Tragweite der klinischen Prüfung einzusehen und seinen Willen hiernach zu bestimmen“ (AMG 1994, § 40 (4) 4.).

Die 12. Novelle des AMG vom 30.07.2004 diente der Umsetzung der europäischen Richtlinie 2001/20/EG auf nationaler Ebene. In den revidierten $\S \S 40$ und 41 wird mehr Gewicht auf die Entscheidung des Kindes oder Jugendlichen über seine Teilnahme an einem klinischen Versuch gelegt. Die Einwilligung des gesetzlichen Vertreters zur Versuchsteilnahme „,muss dem mutmaßlichen Willen des Minderjährigen entsprechen, soweit ein solcher feststellbar ist" (AMG 2004, § 40 (4) 3.). Der Minderjährige müsse seinem Alter und seiner geistigen Reife angemessen über die Arzneimittelprüfung, deren Risiken und Nutzen aufgeklärt werden. Erkläre er, „,nicht an der klinischen Prüfung teilnehmen zu wollen, oder bringt er dies in sonstiger Weise zum Ausdruck, so ist dies zu beachten. Ist der Minderjährige in der Lage, Wesen, Bedeutung und Tragweite der klinischen Prüfung zu erkennen und seinen Willen hiernach auszurichten, so ist auch seine Einwilligung erforderlich“(AMG 2004, §40 (4) 3.).

Die klinische Prüfung an Erwachsenen oder andere Forschungsmethoden dürfen ,keine ausreichenden Prüfergebnisse erwarten lassen“ (AMG 2004, § 40 (4) 2.), und die klinische Prüfung darf nur dann durchgeführt werden, wenn sie „mit möglichst wenig Belastungen und anderen vorhersehbaren Risiken verbunden ist; sowohl der Belastungsgrad als auch die Risikoschwelle müssen im Prüfplan eigens definiert und vom Prüfer ständig überprüft werden“ (AMG 2004, § 40 (4) 4.).

Für eine Versuchsteilnahme dürfen ,Vorteile mit Ausnahme einer angemessenen Entschädigung“ nicht gewährt werden (AMG 2004, § 40 (4) 5.).

Das AMG erlaubt unter bestimmten Bedingungen Forschung an Minderjährigen ohne Eigennutzen. Dabei wird vorausgesetzt, dass die Forschung ,für die Gruppe der Patienten, die an der gleichen Krankheit leiden wie die betroffene Person," von Bedeutung ist (AMG 2004, § 41 (2) 2.a). Diese Forschung dürfe ,für die betroffene Person nur mit einem minimalen Risiko oder einer minimalen Belastung"verbunden sein (AMG 2004, § 41 (2) 2.d). Forschung weise dann ein minimales Risiko auf, wenn zu erwarten sei, ,dass sie allenfalls $\mathrm{zu}$ einer sehr geringfügigen und vorübergehenden Beeinträchtigung der Gesundheit der betroffenen Person führen wird; sie weist eine minimale Belastung auf, wenn zu erwarten ist, dass die Unannehmlichkeiten für die betroffene Person allenfalls vorübergehend auftreten und sehr geringfügig sein werden“(AMG 2004, § 41 (2) 2.d). 


\subsubsection{Medizinproduktegesetz ${ }^{10}$}

Die Ausführungen des Medizinproduktegesetzes von 2002 zur „klinischen Prüfung bei Minderjährigen“ (MPG 2002) in § 20 „Allgemeine Voraussetzungen“ beziehen sich zur Prüfung von Diagnostika und Prophylaktika einerseits auf den gesunden Minderjährigen $^{11}$, andererseits verlangen sie eine persönliche Indiziertheit des Prüfproduktes. Die Frage nach dem zumutbaren Risiko bleibt dabei unberücksichtigt (§ 20 (4) 1.-4.).

Dieses Gesetz entspricht im Wesentlichen den Vorgaben zur Forschung an Minderjährigen in $§ 40$ (4) 1.-4. der Fassung des Arzneimittelgesetzes von 1994.

\subsubsection{Strahlenschutzverordnung, Röntgenverordnung}

Die Strahlenschutzverordnung von 2001 nimmt nicht speziell zur Forschung an Minderjährigen Stellung. Allgemein wird die Anwendung radioaktiver Stoffe oder ionisierender Strahlung an geschäftsunfähigen oder beschränkt geschäftsfähigen Personen geregelt (Strahlenschutzverordnung 2001). ${ }^{12}$ Die Röntgenverordnung von 2003 enthält zu dieser Problematik einen Paragraphen in nahezu gleichem Wortlaut (Röntgenverordnung 2003, § 28d (4)).

\subsubsection{Stellungnahme der ZEKO von 1997}

Die Zentrale Kommission zur Wahrung ethischer Grundsätze in der Medizin und ihren Grenzgebieten (ZEKO) ${ }^{13}$ veröffentlichte 1997 eine Stellungnahme zum „Schutz nicht-

\footnotetext{
${ }^{10}$ Medizinprodukte sind Instrumente, Apparate, Vorrichtungen, Stoffe und Zubereitungen aus Stoffen oder andere Erzeugnisse, die für medizinische Zwecke, wie die Erkennung, Verhütung, Überwachung, Behandlung oder Linderung von Krankheiten, bestimmt sind und deren Hauptwirkung im oder am menschlichen Körper - im Gegensatz zu den Arzneimitteln - nicht auf pharmakologischem, immunologischem oder metabolischem Weg erreicht wird (Bundesinstitut für Arzneimittel und Medizinprodukte 2005).

${ }^{11}$ „Das Arzneimittel muss zum Erkennen oder zum Verhüten von Krankheiten bei Minderjährigen bestimmt sein“" ( 20 (4) 1.).

${ }^{12}$ Im Text heißt es: „An geschäftsunfähigen und beschränkt geschäftsfähigen Probanden ist die Anwendung radioaktiver Stoffe oder ionisierender Strahlung nur zulässig, wenn 1. das Forschungsziel anders nicht erreicht werden kann, 2. die Anwendung gleichzeitig zur Untersuchung oder Behandlung des Probanden angezeigt ist und 3. der gesetzliche Vertreter oder der Betreuer seine Einwilligung abgegeben hat, nachdem er von dem das Forschungsvorhaben leitenden Arzt über Wesen, Bedeutung, Tragweite und Risiken aufgeklärt worden ist. Ist der geschäftsunfähige oder beschränkt geschäftsfähige Proband in der Lage, Wesen, Bedeutung und Tragweite der Anwendung einzusehen und seinen Willen hiernach zu bestimmen, ist zusätzlich dessen persönliche Einwilligung erforderlich“ ( 88 (4)).

${ }^{13}$ Die ZEKO ist ein 1994 vom Vorstand der Bundesärztekammer gegründetes Organ zur Beratung ethischer Grundsatzfragen. Heute repräsentieren 16 Mitglieder die wissenschaftlichen Fachgebiete Medizin, Natur-, Sozial- und Rechtswissenschaften, Philosophie und Theologie. Sie werden für eine Amtsperiode von drei Jahren vom Vorstand der Bundesärztekammer berufen. Die Aufgaben der ZEKO sind
} 
einwilligungsfähiger Personen in der medizinischen Forschung“ (Stellungnahme der ZEKO 1997). Hierin forderte sie für Forschung an nichteinwilligungsfähigen Personen, die nicht im Rahmen eines Heilversuchs stattfindet, dass

- „das Forschungsprojekt nicht auch an einwilligungsfähigen Personen durchgeführt werden kann,

- das Forschungsprojekt wesentliche Aufschlüsse zur Erkennung, Aufklärung, Vermeidung oder Behandlung einer Krankheit erwarten lässt,

- das Forschungsprojekt im Verhältnis zum erwarteten Nutzen vertretbare Risiken erwarten lässt,

- der gesetzliche Vertreter eine wirksame Einwilligung in die Maßnahme erteilt hat, wobei vorausgesetzt ist, daß er aus der Kenntnis der vertretenen Person ausreichende Anhaltspunkte hat, um auf ihre Bereitschaft zur Teilnahme an der Untersuchung schließen zu können,

- ein ablehnendes Verhalten des Betroffenen selbst nicht vorliegt,

- die zuständige Ethikkommission das Forschungsvorhaben zustimmend beurteilt hat" (Abschnitt III).

Bei Forschung ohne Nutzen für die Versuchsperson, aber mit potentiellem Nutzen für Personen der gleichen Alters- und Krankheitsgruppe galt zudem als Voraussetzung,

- „dass das Forschungsprojekt allenfalls minimale Risiken oder Belästigungen erwarten lässt" (Abschnitt III).

\subsubsection{Stellungnahme der ZEKO von 2004}

2004 publizierte die ZEKO eine Stellungnahme zur „Forschung mit Minderjährigen“ (Stellungnahme der ZEKO 2004). Diese unterscheidet drei Typen von Forschung an Minderjährigen: Forschung mit Eigennutzen, Forschung mit Gruppennutzen und Forschung mit reinem Fremdnutzen. Es werden drei durch Beispiele konkretisierte Kategorien von Risiko und Belastung vorgegeben (minimale und mehr als minimale, nämlich niedrige sowie mehr als niedrige Risiken bzw. Belastungen).

Voraussetzungen für eine Einbeziehung von Minderjährigen in gruppennützige Forschung seien ,eine sorgfältige Ermittlung und Bewertung des möglichen Nutzens, eine sorgfältige

die Beurteilung grundsätzlicher ethischer Fragen, die durch den medizinischen Fortschritt und technologische Entwicklungen in der Medizin und ihren Grenzgebieten aufgeworfen werden, die Beurteilung ethischer Fragen, die für die Pflichten bei der Ausübung des ärztlichen Berufes von großer Bedeutung sind, und die Erarbeitung ergänzender Beurteilungen zu ethischen Grundsatzfragen (Klinkhammer 2003, ZEKO 2005). 
Ermittlung und Bewertung möglicher Risiken und Belastungen und das Fehlen milderer Alternativen“" (Abschnitt 4.3).

Risiken und Belastungen der rein fremdnützigen Forschung dürften nicht größer als minimal sein. Bei Forschung mit Aussicht auf einen Gruppennutzen könne in Einzelfällen auch ein niedriges Risiko oder eine niedrige Belastung in Kauf genommen werden. Placeboanwendung sei bei Forschung an Kindern und Jugendlichen nur als Add-on-Therapie und bei fehlender wirksamer Standardtherapie vertretbar.

Der Beteiligung des Minderjährigen an Entscheidungen über seine Versuchsteilnahme misst die ZEKO eine große Bedeutung bei. Die Einwilligung der Eltern sei unverzichtbar, der Minderjährige solle aber ,,seinem Verständnis gemäß so weit wie möglich in die Entscheidungsfindung" einbezogen werden (Abschnitt 4.8). Willensäußerungen wie Irritationen, Abwehr, Angst im situativen Kontext der Durchführung eines Forschungsvorhabens „sollten als Abbruchkriterien definiert werden“ (Abschnitt 4.8).

\subsubsection{Zusammenfassung}

Forschung an Kindern und Jugendlichen wird in allen nationalen und internationalen Empfehlungen sowie auch im aktuellen AMG und MPG an die Bedingung geknüpft, dass sie nicht alternativ an Erwachsenen durchgeführt werden kann. Außerdem wird die Einwilligung der Eltern bzw. der gesetzlichen Vertreter nach erfolgter Aufklärung sowie in unterschiedlichem Maß die Beteiligung des Minderjährigen an Entscheidungen über seine Versuchsteilnahme vorausgesetzt.

Einige hier zitierte Empfehlungen und das AMG in der neuen Fassung machen die Zulässigkeit von Forschung an Kindern und Jugendlichen vom Grad des Risikos und der Belastung abhängig. Dabei werden unterschiedliche Kategorien von Risiko und Belastung als Maß für die Zulässigkeit genannt. Eingegrenzt werden das zulässige Risiko und zum Teil auch die zulässige Belastung bei Forschung ohne potentiellen direkten Eigennutzen $^{14}$ u. a. im aktuellen AMG und in der Stellungnahme der ZEKO von 2004 durch die Begriffe minimales Risiko und minimale Belastung.

Diese rechtlichen und standesethischen Bedingungen für die Zulässigkeit von Forschung an Kindern und Jugendlichen basieren auf der ethischen, pädiatrischen und juristischen Diskussion der letzten Jahre. Im folgenden Kapitel wird diese Diskussion im Hinblick auf die Begriffe Nutzen, Risiko und Belastung, sowie Beteiligung des Minderjährigen an Entscheidungen über seine Versuchsteilnahme zusammenfassend dargestellt.

\footnotetext{
${ }^{14}$ Wenn im Folgenden vom Nutzen eines Forschungsprojektes gesprochen wird, so ist immer sein potentieller Nutzen gemeint, da Forschung ergebnisoffen ist und immer die Möglichkeit besteht, dass Nutzenerwartungen nicht erfüllt werden.
} 


\subsection{Charakterisierung medizinethischer Begriffe}

\subsubsection{Nutzen}

Der Begriff des Nutzens einer Studie ist komplex. Er spielt eine wichtige Rolle bei der Beurteilung der Zulässigkeit von Forschungsprojekten an Kindern und Jugendlichen.

Forschung kann einen therapeutischen Nutzen für die Versuchsperson versprechen. Dieser „Nutzen für die eine betroffene individuelle Versuchsperson“ (Maio 2001b, S. 181), so Giovanni Maio, sei der nächstliegende Grund für Forschung am Menschen. Andere Forschungsprojekte streben für den einzelnen Versuchsteilnehmer während des Versuchs keinen direkten Eigennutzen an. Dieser Versuchstyp geht mit besonderen ethischen Fragestellungen einher. Inwiefern werden Kinder und Jugendliche durch eine Studienteilnahme instrumentalisiert, von der sie nicht unmittelbar persönlich profitieren können?

Eine Debatte zu diesem Thema wurde schon in den siebziger Jahren des 20. Jahrhunderts u. a. von den Theologen und Ethikern Paul Ramsey und Richard McCormick geführt. Ramsey lehnte sogar jede Forschung an einwilligungsunfähigen Minderjährigen ab, da Eingriffe dieser Art ohne persönliche Einwilligung grundsätzlich unzulässig seien. McCormick dagegen machte Forschung an Kindern und Jugendlichen davon abhängig, ob die Minderjährigen zustimmen würden, wenn sie könnten. Bei Versuchen, die einen großen Nutzen für das Allgemeinwohl versprachen und kein erkennbares Risiko erwarten ließen, hielt er auch Forschung ohne direkten Eigennutzen für gerechtfertigt und betrachtete sie sogar als moralische Verpflichtung (Ramsey 1977a, Ramsey 1977b, McCormick 1976, Ramsey 1976).

Ramseys Argumente für eine pauschale Ablehnung der Forschung an Kindern und Jugendlichen werden heute von keinem Ethiker mehr vertreten. Diskutiert wird aber nach wie vor die Möglichkeit einer Rechtfertigung von Forschung ohne direkten Eigennutzen für die minderjährige Versuchsperson. Solche Forschung ist z. B. nötig zur Erhebung von Normalwerten an gesunden Minderjährigen.

Bisher wurden verschiedene Argumente für die Zulässigkeit dieses Typs von Forschung vorgebracht. Eine Position beruft sich auf das Interesse der Allgemeinheit an solchen Versuchen und hält eine geringfügige oder partielle Instrumentalisierung von Minderjährigen für gerechtfertigt (Maio 2001a, Maio 2001b). Eine andere Position betont die moralische Verpflichtung von Kindern und Jugendlichen zur Versuchsteilnahme auch unter dem Gesichtspunkt, dass die Minderjährigen von heute von den Ergebnissen der Forschung an Kindern in der Vergangenheit profitieren (Brock 1994).

Es gibt unterschiedliche Kategorisierungen von Nutzen. Beispielsweise kategorisiert die ZEKO in ihrer Stellungnahme von 2004 Forschung mit reinem Eigen-, mit Gruppen- oder mit Fremdnutzen. Problematisch an diesen Kategorien ist, dass der Unterschied zwischen 
gruppen- und fremdnütziger Forschung bei Forschungsprojekten, deren Nutzen erst in der Zukunft zu erwarten ist, schwierig zu fassen ist. Wer kann absehen, ob Forschungsergebnisse ,,auf zukünftige Personengruppen übertragbar sind“ (Gruppennutzen) (Stellungnahme der ZEKO 2004, 2.3) oder ob es sich nur um Forschung mit „Aussicht auf eine spätere klinische Bedeutung“ (Fremdnutzen) (Stellungnahme der ZEKO 2004, 2.3) handelt? Die Zuordnung zu einer dieser Kategorien entscheidet aber über die Zulässigkeit eines Forschungsprojektes mit größerem als minimalem, niedrigem Risiko (Stellungnahme der ZEKO 2004).

Plausibler erscheint eine Kategorisierung, die im aktuellen AMG vertreten wird. Hier wird bei der Arzneimittelforschung an Minderjährigen in solche mit Eigennutzen (AMG 2004, $\S 40$ (4) 1.) und mit Nutzen ,für die Gruppe der Patienten, die an der gleichen Krankheit leidet wie die betroffene Person," (AMG 2004, § 41 (2) 2. a) unterschieden. Arzneimittelforschung ohne Eigennutzen ist unter der Voraussetzung erlaubt, dass die Forschung ,nur mit einem minimalen Risiko und einer minimalen Belastung verbunden“" ist (AMG 2004, $\S 41(2) 2 . d)$.

Am Beispiel der Krankheitsursachenforschung bei der Fanconi-Anämie verdeutlicht Traute M. Schroeder-Kurth das „Problem der Entscheidung über die vorwiegenden Forschungsziele" (Schroeder-Kurth 2003, S. 291) bei der Zuordnung von Forschungsprojekten zu den unterschiedlich bewerteten Kategorien des Nutzens. Stehe bei der Forschung der mögliche individuelle Nutzen, z. B. im Hinblick auf die Sicherung der Prognose einer Krankheit, im Vordergrund, so werde der Nutzen als das Ziel angesehen und das Forschungsprojekt würde als ethisch gerechtfertigt gelten. Stehe dagegen der wissenschaftliche Erkenntnisgewinn im Vordergrund, so sei dasselbe Projekt als nicht eigennützig zu kategorisieren und bei größerem als minimalem Risiko nicht zulässig. So entscheiden letztlich subjektive Bewertungen von Forschungsprojekten über die ethische und rechtliche Beurteilung durch Ethikkommissionen (Schroeder-Kurth 2003).

\subsubsection{Placeboforschung}

Im Zusammenhang mit der Frage nach dem Nutzen einer Studie wird diskutiert, ob Forschung mit Einsatz eines Placebos sowie Arzneimittelstudien zur Erprobung eines noch nicht zugelassenen Medikaments als Fälle von Forschung ohne direkten Eigennutzen zu gelten haben und wie sie bei Kindern und Jugendlichen zu rechtfertigen sind.

Die Testung neuer Medikamente gegen eine etablierte Standardtherapie oder gegen ein Placebo gehört zum Standard kontrollierter klinischer Studien. Die Anwendung von Placebos ist eine effektive Methode, um subjektive und verfälschende Einflüsse auf Studienergebnisse zu minimieren. Problematisch ist jedoch der Einsatz von Placebopräparaten in der Forschung an Kindern und Jugendlichen als eine besonders schutzbedürftige Gruppe. Ist es vertretbar, Minderjährigen für den Studienzeitraum ein neues Medikament 
oder eine Standardtherapie vorzuenthalten, von der sie vielleicht profitieren könnten und sie nicht zu behandeln? ${ }^{15}$

Durch placebokontrollierte Studien wurden bei Erwachsenen nicht nur wirkungslose Therapien, sondern auch lebensbedrohliche Therapiefolgen, teils von Medikamenten, die bereits auf dem Arzneimittelmarkt regulär verordnet wurden, identifiziert. ${ }^{16}$ Die Gabe von Placebo im Rahmen einer Studie ist auch für die betroffenen pädiatrischen Patienten nicht grundsätzlich nachteilig (Walter-Sack und Haefeli 2001). Nur durch eine Placebostudie konnte beispielsweise festgestellt werden, dass Sumatriptan, das beim Erwachsenen zur Standardtherapie der Migräne gehört, bei Kindern nicht wirksamer ist als Placebo (Hämäläinen et al. 1997).

1983 empfahl Sumner J. Yaffe, Placebos nur dann in pädiatrischen Medikamentenstudien einzusetzen, wenn keine Standardtherapie bekannt sei oder die Wirksamkeit und Sicherheit des neuen Medikaments sich nicht durch Vergleich mit dem natürlichen Krankheitsverlauf ermitteln ließen. Placeboforschung in der Pädiatrie sei auch gerechtfertigt, um ein bereits auf dem Arzneimittelmarkt verfügbares Medikament mit fraglichem Nutzen oder häufigen starken Nebenwirkungen zu überprüfen, sowie bei Krankheiten, für die Spontanheilungen oder plötzliche Verschlimmerungen charakteristisch seien (Yaffe 1983).

Eine ähnlich Auffassung vertritt die ZEKO in ihrer Stellungnahme von 2004. Placeboanwendung sei in der Forschung an Kindern und Jugendlichen nur als Add-on-Therapie und bei fehlender wirksamer Standardtherapie vertretbar. Nicht vertretbar sei es, auch bei leichten, subjektiv stark beeinflussbaren Krankheitszuständen einem Minderjährigen um den Preis einer Placebogabe eine wirksame Therapie vorzuenthalten. Bei umfassend aufgeklärten Erwachsenen sei diese Maßnahme möglicherweise vertretbar (Stellungnahme der ZEKO 2004).

\subsubsection{Risiko und Belastung}

Der Nutzen einer Studie muss gegen die damit verbundenen Risiken und die Belastung für die Versuchsperson abgewogen werden. Welcher Grad von Risiko und Belastung ist vertretbar, wenn an Kindern geforscht wird? Welche Kategorisierungsmöglichkeiten helfen hier weiter? Wie werden das in Gesetzen und Empfehlungen als Schwellenwert für erlaubte Forschung ohne direkten Eigennutzen genannte minimale Risiko und die minimale Belastung definiert und eingegrenzt?

${ }^{15}$ In diesem Zusammenhang ist auch die Problematik der Randomisierung wichtig (Dahl et al. 2002, Snowdon et al. 1998).

${ }^{16}$ Zum Beispiel zeigte 1989 die CAST-Studie (Cardiac Arrythmia Suppression Trial), dass Antiarrhythmika der Klasse I, insbesondere der Klasse Ic, z. B. Flecainid, die Prognose von Patienten nach Myokardinfarkt verschlechtern (Herold 2004). 
Das US-amerikanische Department of Health and Human Services (DHHS) definierte 1974 minimales Risiko folgendermaßen: „Minimales Risiko bedeutet, dass die zu erwartende Wahrscheinlichkeit und Bedeutung einer Schädigung oder Unannehmlichkeit durch einen Versuch nicht größer ist als die Risiken, denen man gewöhnlicherweise im täglichen Leben begegnet, oder die Risiken von körperlichen Routineuntersuchungen oder psychologischen Tests. " 17 Neben objektiven medizinischen Risiken werden in dieser Definition auch subjektive Unannehmlichkeiten berücksichtigt. Als mit minimalem Risiko behaftet werden z. B. Routineimpfungen, nicht invasive körperliche Untersuchungen, Veränderungen der Essgewohnheiten und des Tagesablaufs, die Gewinnung von zusätzlichen Blutproben im Rahmen einer ohnehin notwendigen Blutentnahme und die nicht invasive Gewinnung von Urinproben angesehen (Levine 1989).

Ähnlich beschrieb die American Academy of Pediatrics in ihren Richtlinien minimales Risiko als das Risiko, dem Kinder bei täglichen Aktivitäten ausgesetzt sind. Beispiele für Eingriffe mit minimalem Risiko seien solche, deren Risiko vergleichbar sei mit der Gefahr eines Kindes, beim täglichen Spielen zu stürzen und sich eine Prellung zuzuziehen, also etwa körperliche Untersuchungen, venöse Blutentnahmen und das Sammeln einer Urinprobe (American Academy of Pediatrics Committee on Drugs 1995).

Die Ethikerin Loretta Kopelman kritisierte die Definition des minimalen Risikos der amerikanischen DHHS-Richtlinien kritisierte als inadäquat und unpräzise. Sie sei insofern problematisch, da unklar sei, wessen tägliches Risiko als Maßstab genommen werde. Ein Extremsportler gehe mit anderen Risiken um als jemand, der sich von solchen Beschäftigungen fernhielte. Auch der Begriff Routineuntersuchungen sei nicht sinnvoll, da erstens Routineuntersuchungen für viele Patienten eine Angstquelle seien und zweitens unter diesem Begriff auch riskante Prozeduren wie Röntgenuntersuchungen, Bronchoskopien und Herzkatheteruntersuchungen subsumiert würden (Kopelman 1989).

Eine weitere Kritik an der Definition der DHHS-Richtlinien lautete, dass unklar bleibe, ob es sich bei den Risiken des täglichen Lebens um die Risiken kranker oder gesunder Kinder handele (Freedman et al. 1993, Nelson 1998). Robert Levine, der als Mitglied der National Commission maßgeblich an der Formulierung der zusätzlichen DHHS-Richtlinien „Protections for Children Involved as Subjects in Research“ beteiligt war, betonte 1989 die Notwendigkeit, die Prozeduren, die einer Versuchsperson im Rahmen klinischer Forschung zugemutet werden sollten, in den Kontext ihrer aktuellen und zu erwartenden medizinischen, psychischen oder sozialen Situation einzuordnen. Beispielsweise sei einem an Leukämie leidenden Kind, das im Rahmen der Therapie sowieso mehrere Knochenmarkpunktionen hinter und vor sich habe, durchaus zuzumuten, eine weitere zu Forschungszwecken auf sich zu nehmen. Bei einem gesunden Kind sei es viel problema-

\footnotetext{
17 "Minimal risk means that the probability and magnitude of harm and discomfort anticipated in the research are not greater in and of themselves than those ordinarily encountered in daily life or during the performance of routine physical or psychological examinations or tests" (45 CFR 46 1974, \$46.102 (i)), deutsche Übersetzung: K. E. Radenbach.
} 
tischer, einen solchen Eingriff zu rechtfertigen (Levine 1989). Die gleiche Auffassung vertrat Benjamin Freedman. Risiken könnten nur im individuellen Kontext beurteilt werden (Freedman et al. 1993).

Robert Levine betonte außerdem, dass es Aufgabe der Institutional Review Boards ${ }^{18}$ sei, während der Bearbeitung individueller Studien praxisrelevante Definitionen zum Risikobegriff zu entwickeln (Levine 1989). In einem 1993 erschienenen Artikel „In Loco Parentis. Minimal Risk as an Ethical Threshold for Research upon Children" beschäftigten sich Freedman et al. ebenfalls mit der Verantwortung der Institutional Review Boards bei der Bewertung von Studienprojekten. Die Autoren schlugen vor, Mitglieder von Ethikkommissionen sollten anstelle von Eltern - in loco parentis - über Studien an Minderjährigen entscheiden. Ein grundsätzliches Verbot von Forschung mit unbekanntem Risiko wirke sich langfristig nachteilig für alle Kinder aus. Auch im Alltag ihrer Kinder akzeptierten Eltern gewisse Risiken, um ihnen neue Erfahrungen zu ermöglichen (Freedman et al. 1993).

2000 drängte Loretta Kopelman nochmals darauf, Ethikkommissionen die Beurteilung von Forschungsprojekten durch bessere Definitionen oder Beispiele der Risiken der alltäglichen medizinischen Prozeduren zu erleichtern. Dazu regte sie eine verstärkte öffentliche Diskussion an. Ethikkommissionen sollten multidisziplinär arbeiten, die Öffentlichkeit repräsentieren und sich dem Wohl der Allgemeinheit verpflichtet fühlen. Entscheidungsträger sollten sich in die Situation von Eltern versetzen und überlegen, ob sie genauso entscheiden würden, wenn es um ihr eigenes Kind ginge (Kopelman 2000).

In Großbritannien wurden 1980 in den ersten publizierten Richtlinien der British Paediatric Association zur Forschung an Minderjährigen die drei Kategorien vernachlässigbares Risiko, minimales Risiko und größer als minimales Risiko unterschieden (British Paediatric Association 1980). Später wurden diese Begriffe den US-amerikanischen Richtlinien angepasst, und im Jahr 2000 minimales Risiko, niedriges und hohes Risiko (minimal risk, low risk and high risk) voneinander abgegrenzt (McIntosh et al. 2000). Als mit minimalem Risiko behaftet gelten Prozeduren wie Befragungen, nicht invasive Messungen und Beobachtungen eines Kindes, das nicht invasive Sammeln einer Urinprobe und die Analyse von Blut, das im Rahmen einer ohnehin notwendigen Blutentnahme entnommen wurde. Mit niedrigem Risiko behaftet seien beispielsweise medizinische Maßnahmen, die einen kurzzeitigen Schmerz, Blutergüsse oder kleine Wunden verursachen. Venöse Punktionen und Injektionen gehörten eher in den Bereich des niedrigen als des minimalen Risikos. Dem subjektiven Erleben des Kindes wird in dieser Definition also Raum gegeben. Als Prozeduren mit hohem Risiko werden z. B. Leber- und Lungenpunktionen, arterielle Punktionen und Herzkatheteruntersuchungen angesehen. Sie dürften nicht allein zu Forschungszwecken durchgeführt werden.

${ }^{18}$ Institutional Review Boards sind US-amerikanische Einrichtungen zur Prüfung und Überwachung von Forschung am Menschen vergleichbar mit den deutschen Ethikkommissionen (siehe Abschnitt 2.4). 
Wie sehr in den USA das Verständnis des minimalen Risikos in der Praxis variierte, zeigten Jeffrey Janofsky und Barbara Starfield in der 1981 veröffentlichten Untersuchung „Assessment of risk in research on children“. Die Autoren befragten mittels eines Fragebogens Kinderärzte und Direktoren pädiatrischer Forschungseinrichtungen zur Eingrenzung des minimalen Risikos bei der Forschung an Kindern und Jugendlichen. Die Befragten wurden gebeten, aufgrund ihrer klinischen Erfahrungen medizinische Routineuntersuchungen bei Minderjährigen verschiedener Altersgruppen einzuschätzen und in vier vorgegebene Kategorien (kein Risiko, minimales Risiko, geringfügig größeres als minimales Risiko, mehr als geringfügig größeres als minimales Risiko, no risk, minimal risk, minor increase over minimal risk, greater than minor increase over minimal risk) einzuordnen. Die Ergebnisse zeigten eine große Variabilität bei der Einschätzung des Risikos einer medizinischen Routineuntersuchung (Janofsky und Starfield 1981).

Vermehrt wurde in den letzten Jahren auch der Begriff der psychischen Belastung diskutiert. Medizinische Prozeduren können, unabhängig von ihrem Risiko, für ein Kind oder einen Jugendlichen psychisch sehr belastend sein, wenn sie schmerzhaft, angst- oder schambesetzt sind.

Die ZEKO empfahl 1997 in ihrer Stellungnahme „Zum Schutz nicht-einwilligungsfähiger Personen in der medizinischen Forschung", den Begriff des minimalen Risikos durch „die Unterscheidung von Risikostufen und eine Liste von Beispielen“ zu konkretisieren. $\mathrm{Zu}$ unterscheiden sei auch objektivierbares Risiko und subjektive Belastung. Die ZEKO nannte die Magnetresonanztomographie als ein Beispiel für ein Verfahren, das zwar keine objektivierbaren Risiken in sich berge, aber psychisch belastend sein könne. Die Kommission spricht von einem minimalen Risiko, ,,wenn z. B. Körperflüssigkeit oder Gewebe in geringen Mengen im Rahmen von ohnehin notwendigen medizinischen Maßnahmen oder Operationen gewonnen wird und deshalb kein zusätzliches Risiko für den Patienten beinhaltet. Auch bestimmte körperliche Untersuchungen (z. B. Sonographie, transkutane Gewebemessungen etc.) sowie bestimmte psychologische Untersuchungen (z. B. Fragebogen-Interviews, Tests, Verhaltensbeobachtungen) fallen in diese Gruppe“ (Stellungnahme der ZEKO 1997).

Matthias Dahl und Claudia Wiesemann zogen 2001 eine Bilanz zu internationalen Standards der Forschung an Minderjährigen und leiteten daraus Konsequenzen für die Verwendung des Begriffs des minimalen Risikos bei der Kategorisierung von Forschung ab. Die Autoren forderten ,eine Definition und inhaltliche Präzisierung der Begriffe minimales Risiko und minimale psychische Belästigung" (Dahl und Wiesemann 2001, S. 107) in der deutschen Diskussion. Sie empfahlen, Nutzen und Risiko von Forschung nach Häufigkeit, Schweregrad und Zumutbarkeit zu differenzieren. Um die Begriffe des minimalen Risikos und der minimalen psychischen Belastung zu präzisieren und zu definieren, sei es hilfreich, tolerable Risiken anhand konkreter Beispiele zu erläutern und dabei verschiedene Krankheitsbilder und Altersgruppen zu berücksichtigen (Dahl und Wiesemann 2001). 
Dieser Forderung kam die ZEKO in ihrer Stellungnahme zur „Forschung mit Minderjährigen“" von 2004 teilweise nach. Darin gibt sie zwar keine Definitionen von Risiko und Belastung, konkretisiert aber drei Schweregrade dieser Begriffe durch Beispiele:

- Als mit minimalem Risiko oder mit minimalen Belastungen behaftet könnten danach gelten: Allgemeine klinische Beobachtungen, nicht-invasive Untersuchungstechniken, Erhebung morphometrischer und psychometrischer Daten, die nichtinvasive Sammlung von Ausscheidungsprodukten und geringe zusätzliche Blutentnahmen bei ohnehin liegendem Zugang.

- Als in Einzelfällen schon mit niedrigem Risiko oder niedrigen Belastungen behaftet werden genannt: Punktionen peripherer Venen, Ultraschalluntersuchungen und Magnetresonanztomographien.

- Ein mehr als niedriges Risiko oder eine mehr als niedrige Belastung geht nach der ZEKO-Stellungnahme einher mit Punktionen von Arterien, Punktionen des Knochenmarks, Kontrastmitteluntersuchungen und der Anlage von zentralvenösen Kathetern (Stellungnahme der ZEKO 2004).

In allen Empfehlungen wird Risiko qualitativ kategorisiert. Eine quantitative Kategorisierung von Risiko und eine Berechnung der Risiko-Nutzen-Relation wurde zwar von einigen Wissenschaftlern favorisiert (Nicholson 1986), aber noch nicht in die Praxis umgesetzt.

\subsubsection{Beteiligung des Minderjährigen an Entscheidungen über seine Versuchsteilnahme}

Die Beteiligung von Kindern oder Jugendlichen an Entscheidungen über ihre Versuchsteilnahme wird kontrovers diskutiert. Welche Bedeutung haben dabei das Alter und die Lebenssituation eines Minderjährigen, und welche Rolle spielen seine Eltern bzw. gesetzlichen Vertreter? Welches Gewicht soll die Stimme des Minderjährigen bei der Entscheidung über eine Versuchsteilnahme haben? Inwiefern soll eine Versuchsteilnahme, insbesondere bei Forschung ohne direkten Eigennutzen, von seiner Zustimmung ${ }^{19}$ abhängig gemacht werden?

Im englischen Sprachraum wird für die freiwillige, informierte Einwilligung von Personen zu ihrer Teilnahme in klinischen Versuchen der Begriff des informed consent gebraucht. Nach Ruth R. Faden und Tom L. Beauchamp sind zwei Bedeutungen des Begriffs informed consent zu unterscheiden: informed consent als

\footnotetext{
${ }^{19}$ Der Begriff Zustimmung wird im Folgenden im Sinne der Definition des Begriffs assent des DHHS verwendet, die lautet: "Assent means a child's affirmative agreement to participate in research" (45 CFR 46 1974, $\$ 46.402$ (b)).
} 
1. spezielle autonome Autorisation ${ }^{20}$ und als

2. effektive Autorisation, das heißt als standardisierte Verfahrensweise in institutionellen Kontexten. ${ }^{21}$

Informed consent in der ersten Bedeutung beschreiben die Autoren folgendermaßen: “... an informed consent in sense 1 is given if a patient or subject with (1) substantial understanding, (2) in substantial absence by control of others, (3) intentionally (4) authorizes a professional ..." (Faden und Beauchamp 1986, S. 278).

Der informed consent ist demnach die informierte, freiwillige und absichtsvolle Einwilligung einer Person zu ihrer medizinischen Behandlung oder Studienteilnahme. Um informiert und freiwillig in eine Studienteilnahme einwilligen zu können, muss die Versuchsperson durch einen Arzt über die Inhalte der klinischen Prüfung aufgeklärt werden und in der Lage sein, „Wesen, Tragweite und Bedeutung der klinischen Prüfung zu verstehen und seinen Willen danach zu bestimmen“" (Wiesemann und Biller-Andorno 2005, S. 19). Der informed consent ist ein wichtiger Bestandteil klinischer Studien.

Die Verpflichtung von Wissenschaftlern zum Einhalten ethischer Normen bei Experimenten an Menschen wurde schon in der Urteilsbegründung des Nürnberger Ärzteprozesses von 1946/47, dem so genannten Nürnberger Kodex, erwähnt: „Die freiwillige Zustimmung der Versuchsperson ist unbedingt erforderlich“" (zitiert nach Mitscherlich und Mielke 1947, S. 354). Heute legen in Deutschland die nationalen Gesetze sowie nationale wie internationale Empfehlungen fest, dass Forschung am Menschen nur dann durchgeführt werden darf, wenn die Versuchsperson nach umfassender Aufklärung freiwillig in eine Versuchsteilnahme eingewilligt.

In der Diskussion um Forschung an Minderjährigen wurden die damit verbundenen Fragestellungen früher oft pauschal unter die Rubrik der Forschung an Einwilligungsunfähigen subsumiert und dadurch wichtige Aspekte der Diskussion unterbunden. Gefordert wurde nur die informierte Einwilligung ihrer Eltern bzw. gesetzlichen Vertreter. Kinder und Jugendliche können aber weder pauschal als einwilligungsunfähig bezeichnet, noch mit erwachsenen einwilligungsunfähigen Menschen verglichen werden, die in der Regel in der Vergangenheit einmal einwilligungsfähig waren (Wiesemann 2005).

Voraussetzung für den informed consent ist die sachgerechte und angemessene Information über die geplante Studie. Inwiefern aber können Minderjährige Wesen, Tragweite und Bedeutung klinischer Studien verstehen und ihren Willen danach bestimmen? Sind Kinder und Jugendliche ab einem gewissen Alter vielleicht sogar in der Lage, selbständig, ohne Zustimmung der Eltern, in eine Studienteilnahme einzuwilligen? In anderen Bereichen

20 "Informed consent in sense 1 is defined in terms of the conditions of a particular kind of autonomous authorization.” (Faden und Beauchamp 1986, S. 293) Deutsche Übersetzung: K. E. Radenbach.

21 "By contrast an informed consent in sense 2 is defined in terms of effective authorization, where the nature and acceptability of authorizations are established by operative informed consent rules in a particular policy system." (Faden und Beauchamp 1986, S. 293) Deutsche Übersetzung: K. E. Radenbach. 
ihres Lebens wird älteren Jugendlichen große Entscheidungskompetenz zugestanden: 16jährige können in Deutschland heiraten, öffentlich Alkohol konsumieren und erhalten im Fall einer Schwangerschaft das Sorgerecht für ihr Kind. In anderen Bereichen unserer Gesellschaft (Erhalt der Fahrerlaubnis, Wahlrecht) wird überlegt, die Kompetenzen von Jugendlichen unter 18 Jahren zu erweitern.

Kanadische Wissenschaftler publizierten 1998 eine Untersuchung, die die Qualität kindlicher Zustimmung zur Teilnahme an klinischen Studien untersuchte: 18 gesunde Minderjährige ab einem Alter von fünf Jahren nahmen an einer Studie ohne Eigennutzen zu einer Fragestellung aus der Ernährungsmedizin teil. Vor und nach dem eintägigen Versuch wurden die Kinder und Jugendlichen interviewt. Insgesamt zeigte sich, dass das Verständnis für die Bedeutung der Studie bei allen Kindern unter 9 Jahren niedrig oder gering war. Das Wissen um die Details des Versuchsablaufs war bei allen Teilnehmern gering. Die meisten Kinder und Jugendlichen (78\%) im Alter von zehn Jahren und älter waren sich ihres Rechts, jederzeit von der Studienteilnahme zurücktreten zu können, bewusst. Bei den jüngeren Kindern waren sich nur $20 \%$ darüber im Klaren. Insgesamt zeigte sich, dass die Kinder ab einem Alter von etwa neun Jahren die meisten Studieninhalte verstanden. Die Autoren schließen daher aus ihren Ergebnissen, dass Kinder in einem Alter von acht Jahren oder jünger nicht in der Lage sind, sinnvoll ihre informierte Einwilligung zu klinischer Forschung zu geben (Ondrusek et al. 1998). Einige Autoren nennen dafür andere Altersgrenzen, so z. B. das Alter von 14 (Weithorn und Campbell 1982) oder von sieben Jahren (Laor 1987).

Eine britische Forschergruppe führte Interviews mit 14 Minderjährigen im Alter von sieben bis 18 Jahren, die unmittelbar davor über eine Studie mit Eigennutzen zur Gabe von Wachstumshormon bei Minderwuchs aufgeklärt worden waren. 36\% der befragten Kinder und Jugendlichen hatten nach Einschätzung der Forscher den Studieninhalt nicht verstanden. Einen unmittelbaren Zusammenhang zwischen kindlichem Alter und Grad des Verständnisses für die Studie konnten die Autoren jedoch nicht nachweisen (Postlethwaite et al. 1995).

Die britische Sozialwissenschaftlerin Priscilla Alderson zeigte dagegen in ihren Untersuchungen, dass Kinder mit chronischen Krankheiten bereits im Alter von sechs oder sieben Jahren kompetente Entscheidungen zu ihrer eigenen Therapie treffen können (Alderson 1993). Kinder seien nicht unwissend, weil ihnen Fähigkeit oder Wille zur Einsicht fehle, sondern ,weil sie nicht gründlich genug aufgeklärt wurden“ (Alderson 2003, S. 28). Aufklärung solle man als „gegenseitig wirkenden Prozess“ (Alderson 2003, S. 32) betrachten. Die Ausprägung von Kompetenz und Autonomie sei weit mehr abhängig von Kontext und Erfahrungen als von Alter oder Intelligenz (Alderson 2003).

Der Kinder- und Jugendpsychiater Jörg M. Fegert untersuchte in einer Interviewstudie Partizipationsbedürfnisse und -möglichkeiten bei Aufnahme- und Therapieentscheidungen kinder- und jugendpsychiatrischer Patienten. Er konnte zeigen, dass die Ärzte die 
Informations- und Partizipationsbedürfnisse ihrer minderjährigen Patienten unterschätzten. $56 \%$ der Patienten, die im Interview angaben, nicht an ihrer Aufnahmeentscheidung partizipiert zu haben, sagten, es wäre für sie wichtig gewesen, mit zu entscheiden, $27 \%$ hätten keinen Wert auf ihre Beteiligung gelegt. 59\% der Kinder, die im Laufe ihres stationären Aufenthalts eine medikamentöse Behandlung erhielten, gaben an, die Behandlungsentscheidung sei ohne sie getroffen worden (Fegert et al. 2005).

Einige empirische Studien befassen sich mit der Frage, inwiefern Eltern in der Lage sind, stellvertretend für ihre Kinder informiert und freiwillig über deren Studienteilnahme zu entscheiden. Können sie Studienzwecke und -inhalte durchschauen? Das Problem verschärft sich, wenn Kinder oder Jugendliche schwer erkrankt sind und ihre Eltern sich daher in einer emotional sehr belasteten Situation befinden, in der häufig Zeitdruck herrscht. Dieses Thema wurde während der neunziger Jahre kontrovers diskutiert (Eden 1994, McIntosh 1993, Modi 1993, Tobias und Souhami 1993). Oft wurde die Kompetenz der Eltern, in belasteten Situationen im Sinne des informed consent Entscheidungen zu treffen, angezweifelt. $^{22}$

Eine kanadische Studie an 61 Vätern und Müttern, deren Kinder sich auf einer neonatologischen Intensivstation befanden, ergab beispielsweise, dass für Ärzte und Eltern im Aufklärungsgespräch über die Erkrankung des Neugeborenen unterschiedliche Aspekte relevant waren: Während Ärzte die Vermittlung der Diagnose des kranken Neugeborenen in den Vordergrund stellten, waren den Eltern die Prognose und die Information über das konkrete Procedere besonders wichtig (Perlman et al. 1991).

Robert J. Postlethwaite und seine Mitarbeiter führten im Rahmen der oben genannten Studie zur Einwilligungsfähigkeit von Kindern und Jugendlichen auch Interviews mit 30 Müttern und Vätern, deren Kinder an der Studie zur Gabe von Wachstumshormon bei Minderwuchs teilnahmen. $80 \%$ der Eltern zeigten dabei ein gutes Verständnis für Inhalte, Zweck und Ablauf der geplanten Studie (Postlethwaite et al. 1995).

Ein von diesen Resultaten abweichendes Ergebnis ergab eine australische Studie. Mit einem Fragebogen wurden 62 Eltern während und nach der Studienteilnahme ihrer Kinder zur Testung eines neuen Medikaments in der Therapie des Asthma bronchiale befragt. Es zeigte sich, dass ein beträchtlicher Teil der Eltern sich nicht im Klaren war über ihr Recht, vor einer Einwilligung zur Studienteilnahme ihres Kindes umfassend aufgeklärt zu werden, und über ihre Möglichkeit, jederzeit ohne Nachteile von der Studienteilnahme zurück zu treten. Mögliche Risiken, die mit der Einnahme der Studienmedikation einhergingen, waren ihnen zum Teil nicht bewusst. Fast die Hälfte $(48,4 \%)$ der Eltern nahm an, dass die Medikamentenstudie gar kein Risiko für ihr Kind in sich berge (Harth und Thong 1995).

${ }^{22} \mathrm{Zu}$ Problemen bei der Einwilligung führen kann auch die Tatsache, dass Kinder in der Regel der Einwilligung beider Elternteile als gesetzliche Stellvertreter (doppelte Stellvertreterschaft) bedürfen (Dahl und Wiesemann 2005). 
Nach Einschätzung von Wissenschaftlern sind somit auch Eltern nicht immer gut informiert über Studien, in die sie stellvertretend für ihre Kinder einwilligen. Minderjährige scheinen durchaus fähig zu sein, Studieninhalte nachzuvollziehen und ihre Einstellung zu einer Studienteilnahme reflektiert zu äußern. Sie können ihre Zustimmung oder $A b$ lehnung zu einer Studienteilnahme mitteilen. Ob sie aber einwilligungsfähig sind, daher Wesen, Bedeutung und Tragweite eines Eingriffs verstehen und ihren Willen danach ausrichten, muss im Einzelfall geklärt werden. Aus den zitierten Studien wird auch deutlich, dass das Verfahren des informed consent schwer zu standardisieren ist.

In der Vergangenheit wurden Tests entwickelt, mit denen schnell, praktikabel und verlässlich die Einwilligungsfähigkeit eines Versuchsteilnehmers erfasst werden soll. Mit dem MacArthur Competence Assessment Tool for Treatment kann zum Beispiel geprüft werden, ob der Patient oder Proband in der Lage ist, einen geplanten Versuch zu verstehen und basierend auf seiner Einschätzung selbständig eine Entscheidung über eine Teilnahme zu treffen (Grisso und Appelbaum 1998). In der Praxis der Forschung an Kindern und Jugendlichen wird dieser Test bisher allerdings nicht angewendet (Kölch 2003). Die Aufgabe, ohne wesentliche objektivierende Hilfe die Einwilligungsfähigkeit von Versuchsteilnehmern einzuschätzen, liegt demnach bei den behandelnden Ärzten und Wissenschaftlern, die eine Studie durchführen. Die Qualität der schriftlichen Aufklärungsinformationen wird im Rahmen der Beurteilung von Studienprojekten durch die Ethikkommission begutachtet.

\subsubsection{Beeinflussung der Freiwilligkeit der Versuchsteilnahme eines Minderjährigen}

Rechtliche und standesethische Regelungen zur Forschung an Menschen fordern, dass die Entscheidung zur Teilnahme an einer Studie freiwillig erfolgen müsse und legen fest, dass aus der Ablehnung oder vorzeitigen Beendigung einer Versuchsteilnahme kein Nachteil entstehen darf. Dennoch nahmen in der oben beschriebenen kanadischen Studie $75 \%$ der teilnehmenden Kinder und Jugendlichen an, im Fall ihres Rücktritts von der Studie bei den Forschern negative Gefühle auszulösen (Ondrusek et al. 1998). Eine niederländische Studie aus dem Jahr 1998 ergab, dass sich $25 \%$ der Eltern von 230 Kindern, die an einer randomisierten placebokontrollierten Doppelblindstudie zur Testung von Ibuprofen teilgenommen hatten, zu einer Studienteilnahme verpflichtet gefühlt hatten (van Stuijvenberg et al. 1998).

Inwieweit kann die Freiwilligkeit einer Studienteilnahme eines Minderjährigen auch durch Vergünstigungen, wie z. B. eine vermehrte Betreuung durch ärztliches und pflegerisches Personal oder durch finanzielle Anreize, eingeschränkt sein? Sollen Familien für den zeitlichen und finanziellen Aufwand einer Studienteilnahme finanzielle Anreize oder zumindest eine Entschädigung erhalten? Einerseits kann durch sie die Freiwilligkeit der Versuchsteilnahme eines Minderjährigen eingeschränkt werden, andererseits ist eine Auf- 
wandsentschädigung auch eine Möglichkeit, Anerkennung für eine Studienteilnahme auszudrücken und die Eltern bzw. gesetzlichen Vertreter von real entstehenden Kosten zu entlasten. Claudia Wiesemann vertritt die Ansicht, dass Aufwandsentschädigungen sich im Rahmen dessen bewegen sollten, ,was ein Kind von den Eltern für die Erledigung einer zeitaufwändigen und lästigen Aufgabe erhalten würde bzw. was den Eltern tatsächlich an Kosten entstanden ist" (Wiesemann 2005, S. 132). Dieser Standpunkt entspricht den Vorgaben der Richtlinie 2001/20/EG und des deutschen AMG.

\subsection{Ethikkommissionen}

Eine weitere, grundsätzliche Bedingung für die Zulässigkeit jeder Forschung am Menschen ist die Begutachtung geplanter Studien durch eine Ethikkommission und deren positives Votum (siehe auch Abschnitt 2.2). Ethikkommissionen zur Forschung am Menschen prüfen, ob von Forschern geplante medizinische Forschungsprojekte an Menschen ethisch vertretbar und zulässig sind. Dabei werden hohe Ansprüche an die fachliche Kompetenz ihrer Mitglieder gestellt. Im Folgenden wird beschrieben, wie sich deutsche Ethikkommissionen zusammensetzen und wie sie Forschungsprojekte begutachten.

\subsubsection{Geschichte}

Die ersten deutschen Ethikkommissionen zur Forschung am Menschen orientierten sich am Vorbild der Institutional Review Boards in den USA, deren Einrichtung u. a. auf eine 1966 im New England Journal of Medicine publizierte Studie zurückgeht, nach der mehr als jede vierte klinische Studie gravierende ethische Mängel aufwies (Beecher 1966, Just 2002).

Schon 1972 machte die Deutsche Forschungsgemeinschaft erstmals die Vergabe finanzieller Mittel bei der Etablierung eines Sonderforschungsbereichs von der Einrichtung einer Ethikkommission abhängig. Seit 1975 wird auch in der in Tokio revidierten Fassung der Deklaration von Helsinki empfohlen, vor der Durchführung jedes Forschungsvorhabens an Menschen eine unabhängige Ethikkommission zu befragen. 1978 entstand an der westfälischen Wilhelms-Universität Münster die ,erste unabhängige, allgemeine Ethikkommission in Deutschland“ (Just 2002, S. 92). Ein Jahr später, 1979, empfahl der deutsche Fakultätentag die Gründung von Ethikkommissionen zur Forschung am Menschen an allen medizinischen Fakultäten Deutschlands. Gleichzeitig rief der Vorstand der Bundesärztekammer die Landesärztekammern zur Gründung von Ethikkommissionen auf. 1988 wurde die Notwendigkeit der Einrichtung von Ethikkommissionen zur Forschung am Menschen in der (Muster-)Berufsordnung für Ärzte festgehalten (Deutscher Ärztetag 1988) und 1994 im AMG (AMG 1994) und im MPG (MPG 1994) und seit 1995/1996 
in verschiedenen Kammergesetzen der Länder vorgeschrieben. In anderen europäischen Ländern ist eine ähnliche Entwicklung zu beobachten (Deutsch und Lippert 1998, Just 2002, Klinkhammer 2003).

Zur Harmonisierung von Verfahren, Standards und Kriterien der Ethikkommissionen wurde der Arbeitskreis medizinischer Ethikkommissionen gebildet. Die Mitglieder beschließen Verfahrensgrundsätze, die von den einzelnen Ethikkommissionen meist weitgehend übernommen werden (Klinkhammer 2003).

\subsubsection{Aufgaben, Zusammensetzung und Arbeitsweise}

Zentrale Aufgabe jeder Ethikkommission ist die Prüfung und Kontrolle von biomedizinischer Forschung am Menschen. Ethikkommissionen beraten Wissenschaftler nach den Vorgaben von Gesetzen und Empfehlungen, damit deren Forschungsprojekte den Anforderungen zum „Schutz der körperlichen und seelischen Unversehrtheit, der Sicherung des Selbstbestimmungsrechts des Patienten und nach einer Patienteninformation und Einverständniserklärung" genügen (Just 2002, S. 95). Ethikkommissionen handeln im Interesse und zum Schutz der Patienten und Probanden. Gleichzeitig schützen sie durch ihr Votum auch Ärzte und Forschungsinstitutionen vor fehlerhaftem Verhalten und vor Regressansprüchen (Klinkhammer 2003).

In Deutschland sind derzeit etwa 50 öffentlich-rechtliche Ethikkommissionen tätig. Davon sind etwa ein Drittel bei den Landesärzte- oder Landeszahnärztekammern und zwei Drittel an den Universitätskliniken angesiedelt (Just 2002). Die Ethikkommissionen der Universitäten beraten auch Forschungsprojekte aus dem Bereich der Grundlagenforschung, während den Ethikkommissionen der Landesärztekammern fast ausschließlich Arzneimittelstudien vorgelegt werden, bei denen nach $\S 40$ des Arzneimittelgesetzes das positive Votum einer Ethikkommission für die Durchführung vorausgesetzt wird (Klinkhammer 2003).

Eine Ethikkommission setzt sich aus Sachverständigen verschiedener Berufsgruppen zusammen, die ihre Funktion als Mitglied meist ehrenamtlich ausüben. Folgende Berufsgruppen sind in Ethikkommissionen vertreten: Klinisch arbeitende Mediziner, Pharmakologen, Juristen und Biostatistiker, oft auch Medizinethiker, Philosophen, Theologen, Vertreter der Pflegewissenschaften, Medizintechniker, Psychologen, medizinische Laien und andere (Just 2002). Die Mitglieder einer Ethikkommission müssen in der Regel keine besondere medizinethische Qualifikation vorweisen. Im Bedarfsfall werden zu speziellen Themen Sachverständige eingeladen und um eine Stellungnahme gebeten. Die Mitglieder werden vom Vorstand oder der Delegiertenversammlung der Landesärztekammern oder der zuständigen städtischen Behörde bzw. von der medizinischen Fakultät oder dem Senat der Universität ernannt oder gewählt. Eine Amtsperiode dauert meist vier Jahre (Just 2002). 
Forschungsprojekte, die beraten werden, sind medizinische und zahnmedizinische Untersuchungen an Menschen, an körpereigenem Material, entnommenen Körperflüssigkeiten und -geweben, Untersuchungen genetischen Materials, epidemiologische Forschungsvorhaben und Untersuchungen mit Hilfe personenbezogener Daten. Einige Kommissionen beraten auch außerhalb ihrer gesetzlich geforderten Aufgaben ärztliches und nichtärztliches Personal zu ethischen Fragen oder begutachten nicht-medizinische Projekte (Just 2002).

Die Beratungen sind nicht öffentlich. Mitglieder, die an einem Antrag beteiligt oder selbst Antragsteller sind, nehmen nicht an der jeweiligen Beratung teil. In der Regel strebt die Kommission eine Konsensentscheidung an (Just 2002).

Unter anderem durch die Novellierung des Arzneimittelgesetzes von 2004 wurden die Aufgaben von deutschen Ethikkommissionen erweitert und präzisiert. Sie ,sind in diesen Feldern zu einer Art Genehmigungsbehörde geworden“" (Raspe 2005, S. A3166). 


\section{Fragestellung der Arbeit}

Die Bedingungen für die Zulässigkeit von Forschung an Kindern und Jugendlichen sind in Gesetzen und nationalen und internationalen Empfehlungen festgelegt. Sie greifen zurück auf Begriffe der nationalen und internationalen ethischen Diskussion zur Forschung an Minderjährigen.

Alle in Abschnitt 2.2 beschriebenen Gesetze und Empfehlungen verlangen, dass Forschung, die an Kindern und Jugendlichen durchgeführt wird, dem minderjährigen Versuchsteilnehmer persönlich Nutzen verspricht oder zumindest für andere Kinder einen Benefit erwarten lässt. Weiterhin muss ausgeschlossen sein, dass die Prüfung mit vergleichbaren Ergebnissen auch an Erwachsenen durchgeführt werden kann.

\subsection{Risiko und Belastung}

Die Zulässigkeit der Forschung an Kindern und Jugendlichen ohne direkten Eigennutzen für die Versuchsperson wird insbesondere an ihren Grad von Risiko und Belastung geknüpft. Die Gesetze und Empfehlungen charakterisieren das zulässige Risiko und die zulässige Belastung bei Forschungsprojekten, die keinen Eigennutzen anstreben. Eine Übersicht darüber zeigt Tabelle 3.1.

Das Menschenrechtsübereinkommen zur Biomedizin des Europarates, das aktuelle AMG und die Stellungnahme der ZEKO von 1997 verwenden den Begriff des minimalen Risikos bzw. der minimalen Belastung als das nicht zu überschreitende Maß für die Zulässigkeit von Forschung an Kindern und Jugendlichen ohne direkten Eigennutzen. Die Stellungnahme der ZEKO von 2004 erlaubt in Einzelfällen auch gruppennützige Forschung mit niedrigem Risiko.

Die praktische Umsetzung dieser Begriffe soll durch Ethikkommissionen erfolgen, die die entscheidenden Instanzen bei der Beurteilung der Zulässigkeit konkreter Studienpläne sind. Ihre Einschätzung der Zulässigkeit bestimmter Typen der Forschung an Minderjährigen und die daraus resultierenden Schwierigkeiten sind Gegenstand der vorliegenden 


\begin{tabular}{ll} 
Gesetz/Empfehlung & $\begin{array}{l}\text { Zulässiges Risiko/zulässige Belastung bei Forschung } \\
\text { ohne direkten Eigennutzen }\end{array}$ \\
\hline
\end{tabular}

Guidelines des CIOMS “...no more likely and not greater than the risk at(2002) tached to routine medical or psychological examination of such persons. Slight or minor increases above such risk may be permitted." (Guideline 9)

Richtlinie 2001/20/EG Unter Berücksichtigung der Erkrankung und des Ent(2001) wicklungsstadiums möglichst wenig Schmerzen, Beschwerden, Angst und andere vorhersehbare Risiken; Risikoschwelle und Belastungsgrad „müssen eigens definiert und ständig überprüft werden“ (Artikel $4 \mathrm{~g}$ ).

Menschenrechtsüberein- Minimales Risiko und minimale Belastung (Artikommen des Europarates (1997)

AMG (2004)

Minimales Risiko und minimale Belastung (§ 41 (2) 2.d).

Stellungnahme der ZEKO (1997)

Allenfalls minimale Risiken oder minimale Belästigungen (III).

Stellungnahme der ZEKO (2004) Im Regelfall minimale Risiken oder Belastungen (4.6).

Tabelle 3.1: Aussagen von Gesetzen und Empfehlungen zum zulässigen Risiko und zur zulässigen Belastung bei Forschung ohne direkten Eigennutzen 
Arbeit. Dabei werden im Einzelnen folgende Fragen verfolgt:

- Werden bei der Beurteilung der Zulässigkeit von Forschung an Kindern und Jugendlichen ohne direkten Eigennutzen die in den Gesetzen und Empfehlungen festgelegten Bedingungen verwendet?

- Ist der Grad an Risiko und Belastung ein zentrales Kriterium bei der Beurteilung der Zulässigkeit von Forschung an Kindern und Jugendlichen ohne direkten Eigennutzen, so, wie von Gesetzen und Empfehlungen gefordert?

- Orientieren sich Ethikkommissionen bei Entscheidungen für oder gegen Forschungsprojekte ohne direkten Eigennutzen für den teilnehmenden Minderjährigen am Begriff des minimalen Risikos bzw. der minimalen Belastung?

- Wie wird mit Eingriffen umgegangen, deren Risiken und Belastungen mehr als minimal sind?

- Wird die Zulässigkeit von konkreten Forschungsprojekten einheitlich beurteilt?

\subsection{Beteiligung des Minderjährigen an Entscheidungen über seine Versuchsteilnahme}

Voraussetzung für die Durchführung einer Studie an Minderjährigen ist die Einwilligung der Eltern bzw. gesetzlichen Vertreter nach erfolgter Aufklärung. In unterschiedlichem Umfang werden auch Aufklärung und Zustimmung bzw. eine Einwilligung des Kindes oder Jugendlichen gefordert. Eine Übersicht über die Aussagen von Gesetzen und Empfehlungen zur Beteiligung des Minderjährigen an Entscheidungen über seine Versuchsteilnahme zeigt Tabelle 3.2.

Gesetz/Empfehlung

Deklaration von Helsinki (2004)

Guidelines des CIOMS (2002)
Aussagen zur Beteiligung des Minderjährigen an Entscheidungen über seine Versuchsteilnahme

"When a subject deemed legally incompetent, such as a minor child, is able to give assent to decisions about participation in research, the investigator must obtain that assent." (Grundsatz 25)

"... the agreement (assent) of each child has been obtained to the extent of the child's capabilities"; "... a child's refusal to participate or continue in the research will be respected." (Guideline 14) 
Gesetz/Empfehlung

Richtlinie 2001/20/EG

(2001)

Menschenrechtsübereinkommen des Europarates (1997)

AMG (1994)

AMG (2004)

MPG (2002)

Stellungnahme der ZEKO (1997)
Aussagen zur Beteiligung des Minderjährigen an Entscheidungen über seine Versuchsteilnahme

Einwilligung der Eltern muss mutmaßlichem Willen des Minderjährigen entsprechen (Artikel 4a).

Aufklärung des Minderjährigen durch geschultes Personal (Artikel 4b).

Ablehnung soll bei ausreichendem Verständnis der Aufklärungsinformationen berücksichtigt werden (Artikel 4c).

Ansicht des Minderjährigen ,wird als ein mit zunehmendem Alter und entsprechender Reife an Bedeutung gewinnender Faktor berücksichtigt" (Artikel 6(2)).

Schriftliche Einwilligung, wenn Minderjähriger in der Lage ist, „Wesen, Bedeutung und Tragweite der klinischen Prüfung einzusehen und seinen Willen hiernach zu bestimmen“ ( $\$ 40$ (4) 4.).

Einwilligung des gesetzlichen Vertreters muss mutmaßlichem Willen des Minderjährigen entsprechen. Aufklärung gemäß Alter und geistiger Reife.

Ablehnung, durch Erklärung oder ,,in sonstiger Weise zum Ausdruck“" gebracht, soll beachtet werden.

Einwilligung, wenn Minderjähriger in der Lage ist, „Wesen, Bedeutung und Tragweite der klinischen Prüfung zu erkennen und seinen Willen hiernach auszurichten“" (\$ 40 (4) 3.).

Schriftliche Einwilligung, wenn Minderjähriger in der Lage ist, „Wesen, Bedeutung und Tragweite der klinischen Prüfung einzusehen und seinen Willen hiernach zu bestimmen“ (§ 20 (4) 4.).

Aus der Kenntnis der vertretenen Person muss auf ihre Bereitschaft zur Teilnahme geschlossen werden können.

Ablehnendes Verhalten des Betroffenen liegt nicht vor. 


\begin{tabular}{ll}
\hline Gesetz/Empfehlung & $\begin{array}{l}\text { Aussagen zur Beteiligung des Minderjährigen an Ent- } \\
\text { scheidungen über seine Versuchsteilnahme }\end{array}$ \\
\hline $\begin{array}{l}\text { Stellungnahme der ZEKO } \\
(2004)\end{array}$ & $\begin{array}{l}\text { Einbeziehung in Entscheidungsfindung dem „Ver- } \\
\text { ständnis gemäß so weit wie möglich“. } \\
\\
\text { Irritationen, Abwehr, Angst sind Abbruchkriterien } \\
(4.8) .\end{array}$ \\
\hline
\end{tabular}

Tabelle 3.2: Aussagen von Gesetzen und Empfehlungen zur Beteiligung des Minderjährigen an Entscheidungen über seine Versuchsteilnahme

Die Zustimmung (Deklaration von Helsinki 2004, Guidelines des CIOMS 2002) eines Minderjährigen soll, wenn möglich, eingeholt werden. Die Einwilligung (AMG 1994, AMG 2004, MPG 2002, Stellungnahme der ZEKO 2004) eines Kindes oder Jugendlichen in seine Studienteilnahme wird von dessen Fähigkeit, die Studie zu verstehen, abhängig gemacht. Abwehrendes Verhalten soll berücksichtigt werden. Die Richtlinie 2001/20/EG macht die Berücksichtigung einer Ablehnung aber davon abhängig, ob das Kind oder der Jugendliche ,sich eine eigene Meinung bilden kann und die erhaltenen Informationen zu beurteilen weiß“ (Richtlinie 2001/20/EG 2001, Artikel 4 c). Andere Empfehlungen (Guidelines des CIOMS 2002, Stellungnahme der ZEKO 2004) und das AMG von 2004 machen diese Einschränkung nicht und definieren dagegen zum Teil schon unspezifisches Abwehrverhalten wie „Irritation, Abwehr, Angst im situativen Kontext“ (Stellungnahme der ZEKO 2004, 4.8) als Abbruchkriterien.

Der Einstellung eines Minderjährigen zu seiner Studienteilnahme wird in Gesetzen und Empfehlungen in den letzten Jahren ein größerer Stellenwert zugemessen. Gleichzeitig wird die Rolle von Eltern und gesetzlichen Vertretern im Einwilligungsprozess kritisch und differenziert betrachtet (vgl. z. B. Dahl et al. 2002, Snowdon et al. 1998).

Mitglieder von Ethikkommissionen sind in der Regel bei der Durchführung von Studien nicht anwesend. Die konkrete Umsetzung der in Gesetzen und Empfehlungen wiedergegebenen Bedingungen zur Aufklärung und Einwilligung der Eltern bzw. gesetzlichen Vertreter und zur Beteiligung des Minderjährigen an Entscheidungen über seine Versuchsteilnahme im Kontakt mit den Versuchspersonen und ihren Angehörigen liegt in der Hand der Wissenschaftler, die die Studie durchführen. Den Mitgliedern von Ethikkommissionen liegen aber bei der Beurteilung zukünftiger Studien das den Eltern und ihren Kindern auszuhändigende schriftliche Aufklärungsmaterial und die Einwilligungsformulare vor. Außerdem enthält ein Antrag an die Ethikkommission ggf. Angaben zu den Abbruchkriterien der Studie, wie z. B. ein abwehrendes Verhalten des Minderjährigen. 
Ein weiterer Aspekt der Beteiligung des Minderjährigen an Entscheidungen über seine Versuchsteilnahme ist die Beeinflussung der Freiwilligkeit seiner Versuchsteilnahme durch finanzielle oder andere Anreize. Im positiven Sinne kann durch die Zahlung einer Aufwandsentschädigung aber auch Anerkennung für die Versuchsteilnahme ausgedrückt und eine Entschädigung für den damit verbundenen Aufwand geleistet werden.

Die Richtlinie 2001/20/EG, die Guidelines des CIOMS und das AMG von 2004 stellen Bedingungen zur Zulässigkeit der Honorierung einer Versuchsteilnahme. Eine Übersicht darüber zeigt Tabelle 3.3.

\begin{tabular}{ll}
\hline Gesetz/Empfehlung & $\begin{array}{l}\text { Aussagen zur Begrenzung von Anreizen für eine Ver- } \\
\text { suchsteilnahme }\end{array}$ \\
\hline $\begin{array}{l}\text { Guidelines des CIOMS } \\
(2002)\end{array}$ & $\begin{array}{l}\text { “...no recompense other than a refund of travel and } \\
\text { related expenses” (Guideline 7). }\end{array}$ \\
$\begin{array}{l}\text { Richtlinie 2001/20/EG } \\
(2001)\end{array}$ & $\begin{array}{l}\text { Keine Anreize oder finanzielle Vergünstigungen au- } \\
\text { ßer einer Entschädigung (Artikel 4 d). }\end{array}$ \\
AMG (2004) & $\begin{array}{l}\text { Keine Vorteile außer einer angemessenen Entschädi- } \\
\text { gung (\$ } 40(4) 5 .) .\end{array}$ \\
\hline
\end{tabular}

Tabelle 3.3: Aussagen von Gesetzen und Empfehlungen zur Begrenzung von Anreizen

Auch bei der Erfüllung dieser Bedingungen spielen Ethikkommissionen eine wichtige Rolle, da ihnen ggf. auch Angaben zu einer Honorierung einer Versuchsteilnahme vorgelegt werden. Unklar ist nach wie vor, ob und wenn ja, für welche Art von Studien und für Kinder in welchem Alter eine finanzielle Entschädigung gezahlt werden sollte. Die Gesetze und Empfehlungen differenzieren auch nicht, ob Eltern bzw. gesetzliche Vertreter oder das Kind belohnt werden sollen.

Aus diesen Überlegungen ergeben sich weitere Fragen, zu deren Klärung die vorliegende Arbeit beitragen möchte:

- Welche Bedeutung hat die Einstellung eines Kindes oder Jugendlichen zu seiner Studienteilnahme für die Beurteilung der Zulässigkeit eines Forschungsprojektes?

- Wie wichtig ist eine individuelle Aufklärung und Zustimmung von Kindern in unterschiedlichen Altersstufen?

- Wie soll im Fall eines abwehrenden Verhaltens vorgegangen werden?

- Inwiefern sollen einem Minderjährigen oder seinen Eltern bzw. gesetzlichen Vertretern finanzielle Anreize bzw. eine finanzielle Entschädigung für eine Studienteilnahme geboten werden? 


\subsection{Ansatz zur Bearbeitung der Fragen}

Die in Abschnitt 3.1 und 3.2 entwickelten Fragen werden in der vorliegenden Arbeit empirisch untersucht. Dazu wurde ein Fragebogen entwickelt, der mehrere Vignetten zu Forschungsprojekten in der Kinderheilkunde und Jugendmedizin und in der Kinder- und Jugendpsychiatrie auf der Basis authentischer Studienentwürfe enthält. Dieser wurde den Vorsitzenden deutscher Ethikkommissionen zur Beantwortung vorgelegt.

Um die Praxisrelevanz der Fragestellungen zu prüfen, wurden vorab Daten über konkrete Forschungsprojekte an Kindern und Jugendlichen an einer deutschen Universitätsklinik gewonnen. Zu diesem Zweck wurden sämtliche Anträge zur Forschung an Minderjährigen an die Ethikkommission der Georg-August-Universität Göttingen der Jahre 1999 bis 2003 ausgewertet.

In Abschnitt 4 und 5 werden Methodik und Ergebnisse beider Teile des empirischen Projektes dargestellt. Die in der Arbeit verwendeten Begriffe Nutzen, Risiko und Belastung werden im folgenden Abschnitt 3.4 kategorisiert.

\subsection{Kategorisierung}

\subsubsection{Nutzen}

Zur Kategorisierung des Begriffs Nutzen soll zwischen einem direkten Eigennutzen (der Nutzen kann direkt auf die geprüfte Maßnahme zurückgeführt werden) für die minderjährigen Versuchsteilnehmer und einem Nutzen für gleichartig erkrankte Kinder und Jugendliche unterschieden werden. Dabei wird ein Nutzen für gleichartig erkrankte Kinder und Jugendliche mit dem Begriff enger Gruppennutzen bezeichnet. Diese beiden, auch im aktuellen AMG unterschiedenen Kategorien, werden ergänzt durch die Kategorie eines Nutzens für andere Kinder und Jugendliche, im Folgenden erweiterter Gruppennutzen genannt.

\subsubsection{Risiko und Belastung}

Zwei Grade von Risiko und Belastung werden in der vorliegenden Arbeit voneinander abgegrenzt: Minimales Risiko und mehr als minimales Risiko sowie minimale Belastung und mehr als minimale Belastung. Diese Kategorisierung anhand von Beispielen orientiert sich an der internationalen Diskussion zu den Begriffen Risiko und Belastung und an den Stellungnahmen der ZEKO von 1997 und 2004 (siehe Abschnitt 2.3.2). 
Tabelle 3.4 zeigt die in der Arbeit verwendete Kategorisierung von Risiko und Belastung anhand verschiedener Beispiele.

Körperliche Untersuchungen und psychokognitive Tests beinhalten ein minimales Risiko. Ihre Aufnahme in die Kategorie minimale Belastung ist aber an Bedingungen geknüpft (siehe Tabelle 3.4), da sie eine größere als minimale Belastung aufweisen können, wenn sie nicht kindgerecht durchgeführt werden. Die Kategorisierung von Belastung durch „Punktionen aller Art“ und „Entnahme von Gewebe aller Art“ wird davon abhängig gemacht, ob diese Prozedur im Rahmen einer ohnehin notwendigen Vollnarkose geschieht oder nicht. 


\begin{tabular}{|c|c|}
\hline Minimales Risiko & Minimale Belastung \\
\hline $\begin{array}{l}\text { - Allgemeine klinische Beobachtun- } \\
\text { gen } \\
\text { - Körperliche Untersuchungen und } \\
\text { psychokognitive Tests aller Art } \\
\text { (nicht-invasive Untersuchungstech- } \\
\text { niken, z. B. allgemeine klinische } \\
\text { Untersuchung und Sonographie; } \\
\text { Erhebung morphometrischer Da- } \\
\text { ten, Erhebung psychometrischer } \\
\text { Daten) } \\
\text { - Nicht-invasive Sammlung von } \\
\text { Ausscheidungsprodukten } \\
\text { - Geringfügige Blutentnahmen ohne } \\
\text { Notwendigkeit zusätzlicher Punk- } \\
\text { tionen }\end{array}$ & $\begin{array}{l}\text { - Allgemeine klinische Beobachtun- } \\
\text { gen } \\
\text { - Körperliche Untersuchungen und } \\
\text { psychokognitive Tests aller Art } \\
\text { (nicht-invasive Untersuchungstech- } \\
\text { niken, Erhebung morphometrischer } \\
\text { Daten, Erhebung psychometrischer } \\
\text { Daten), wenn sie spielerisch, kind- } \\
\text { gerecht und ohne Verletzung von } \\
\text { Schamgefühlen vonstatten gehen } \\
\text { - Nicht-invasive Sammlung von } \\
\text { Ausscheidungsprodukten } \\
\text { - Geringfügige Blutentnahmen ohne } \\
\text { Notwendigkeit zusätzlicher Punk- } \\
\text { tionen } \\
\text { - Punktionen aller Art (venöse und } \\
\text { arterielle Punktionen, Knochen- } \\
\text { markpunktionen, Lumbalpunktio- } \\
\text { nen u. a.) und Entnahme von Gewe- } \\
\text { be mit sowieso notwendiger Voll- } \\
\text { narkose }\end{array}$ \\
\hline Mehr als minimales Risiko & Mehr als minimale Belastung \\
\hline $\begin{array}{l}\text { - Punktionen aller Art (venöse und } \\
\text { arterielle Punktionen, Knochen- } \\
\text { markpunktionen, Lumbalpunktio- } \\
\text { nen u. a.) } \\
\text { - Entnahme von Gewebe aller Art } \\
\text { - Gabe eines Placebos } \\
\text { - Studienbedingter Verzicht auf } \\
\text { Standardtherapie }\end{array}$ & $\begin{array}{l}\text { - Punktionen aller Art (venöse und } \\
\text { arterielle Punktionen, Knochen- } \\
\text { markpunktionen, Lumbalpunktio- } \\
\text { nen u. a.) ohne Vollnarkose } \\
\text { - Entnahme von Gewebe aller Art } \\
\text { ohne Vollnarkose } \\
\text { - Gabe eines Placebos über einen } \\
\text { längeren Zeitraum } \\
\text { - Häufigere und längere studienbe- } \\
\text { dingte Krankenhausaufenthalte und } \\
\text { dadurch bedingte schulische Fehl- } \\
\text { zeiten }\end{array}$ \\
\hline
\end{tabular}

Tabelle 3.4: Kategorisierung von Risiko und Belastung 


\section{Auswertung von Forschungsanträgen}

\subsection{Methodik}

Wie in Abschnitt 3.3 beschrieben, wurden die Anträge zur Forschung an Kindern und Jugendlichen der Jahre 1999 bis 2003 an die Ethikkommission der Georg-August-Universität Göttingen ausgewertet, um Daten zu konkreten Forschungsprojekten zu gewinnen und die Praxisrelevanz der in Abschnitt 3.1 und 3.2 formulierten Fragen zu überprüfen. In den Jahren von 1999 bis 2003 wurden insgesamt 73 Anträge zur Forschung an Kindern und Jugendlichen an die Ethikkommission Göttingen gestellt.

Die Gesamtzahl aller Anträge betrug in diesem Zeitraum 1100 (Ethikkommission der Georg-August-Universität Göttingen 2005). Der Anteil der eingereichten Anträge zur Forschung an Kindern und Jugendlichen betrug damit 6,6\%.

Abbildung 4.1 zeigt die Anzahl der Anträge zur Forschung an Kindern und Jugendlichen in den einzelnen Jahren von 1999 bis 2003. Sie variiert zwischen 13 (in den Jahren 2000, 2001 und 2002) und 18 Anträgen (im Jahr 2003).

\subsubsection{Erhebung der Daten}

Die Originalanträge zur Forschung an Kindern und Jugendlichen an die Ethikkommission der Georg-August-Universität Göttingen aus den Jahren 1999 bis 2003 wurden in die in Abschnitt 3.4 beschriebenen Kategorien von Nutzen (d.h. direkter Eigennutzen für die Versuchsperson und enger bzw. erweiterter Gruppennutzen) eingeordnet. Darunter gab es auch Anträge, die sowohl engen als auch erweiterten Gruppennutzen enthielten. Diese wurden gesondert aufgeführt.

Den ganz oder teilweise gruppennützigen Anträgen wurde entnommen, ob eine Placebogabe geplant war, da Placeboforschung einen wichtigen Spezialfall der gruppennützigen Forschung darstellt, der auch in den Vignetten berïcksichtigt wird.

Die Risiken und Belastungen durch Eingriffe zu Forschungszwecken in den ganz oder teilweise gruppennützig angelegten Studien wurden anschließend einer der in Abschnitt 
Anträge zu Forschung an Kindern und Jugendlichen

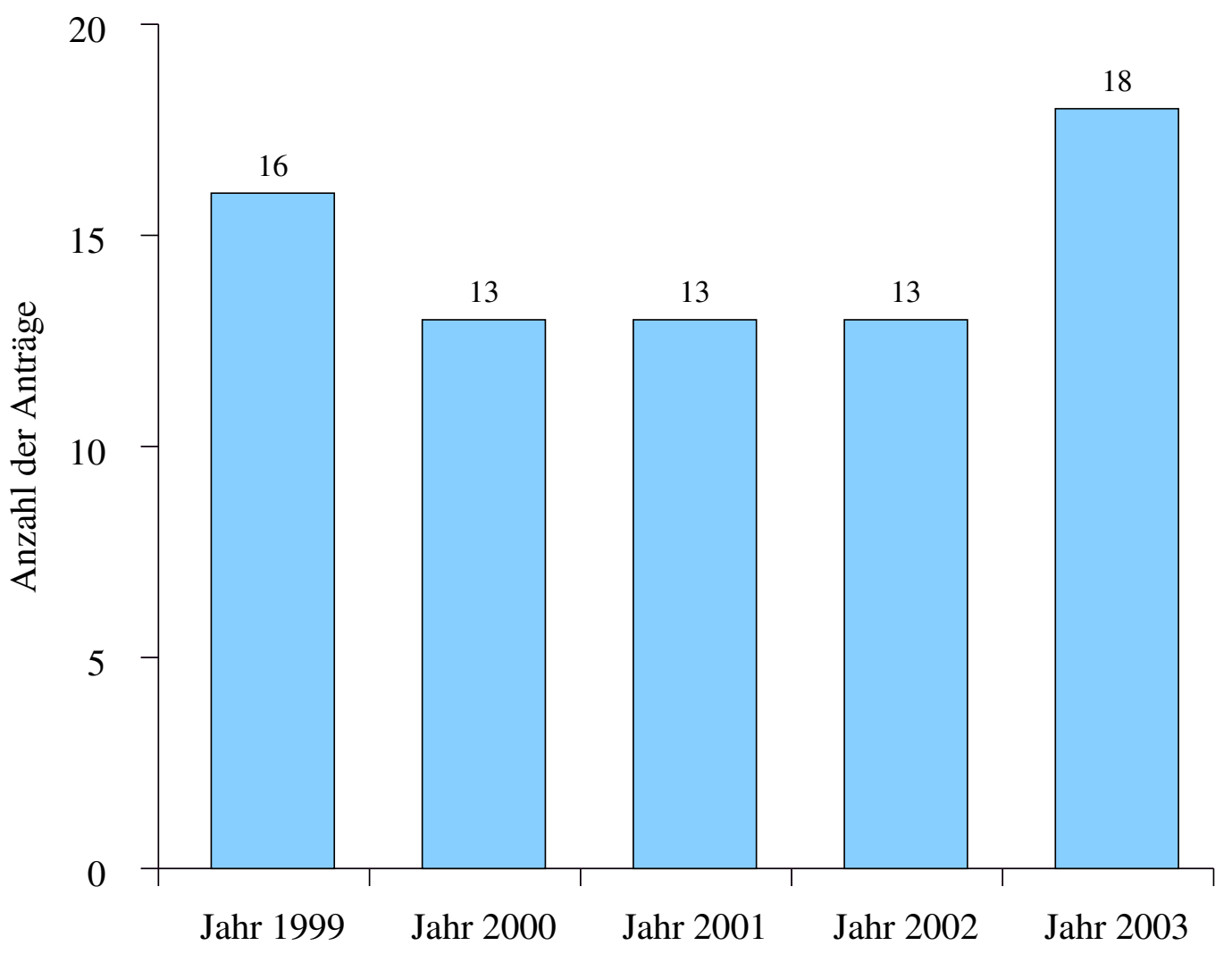

Abbildung 4.1: Anzahl der Anträge zur Forschung an Kindern und Jugendlichen an die Ethikkommission Göttingen 1999-2003 
3.4 beschriebenen Kategorien (d. h. minimales Risiko bzw. mehr als minimales Risiko und minimale Belastung bzw. mehr als minimale Belastung) zugeordnet, um einen Eindruck zu gewinnen, welcher Anteil der gruppennützigen Forschungsprojekte tatsächlich Eingriffe mit mehr als minimalem Risiko und mehr als minimaler Belastung beinhaltete.

Um festzustellen, wie viele der Anträge überhaupt von einer gesetzlichen Regelung erfasst wurden, wurde bei allen Anträgen ermittelt, wo Forschung zu Arzneimitteln oder zu Medizinprodukten vorgesehen war.

Um die Frage nach der Häufigkeit einer finanziellen Entschädigung für eine Studienteilnahme zu beantworten, wurde erfasst, ob die geplanten Studien eine Aufwandsentschädigung vorsahen.

Es wurden ausschließlich Originalanträge ausgewertet, die noch nicht nach Vorgaben der Ethikkommission korrigiert waren, um ein möglichst unverfälschtes Bild der ursprünglichen Anträge und der primären Intention der Forscher zu erhalten.

Die Auswertung der Daten erfolgte computergestützt mit Hilfe des Tabellenkalkulationsprogramms OpenOffice.org Calc.

\subsection{Ergebnisse}

Aus Datenschutzgründen werden hier keine Daten veröffentlicht, die mit einzelnen Abteilungen des Göttinger Klinikums und ihren wissenschaftlichen Projekten in Verbindung gebracht werden können.

\subsubsection{Nutzen der Studienprojekte}

Abbildung 4.2 zeigt den Anteil von Studien mit direktem Eigennutzen, mit engem und mit erweitertem Gruppennutzen und mit Anteilen von beiden Kategorien des engen und erweiterten Gruppennutzens.

16 geplante Studien $(21,9 \%)$ hatten einen Ansatz mit direktem Eigennutzen für die Versuchsperson. In 34 Studien (46,6\%) sollte an kranken Kindern ohne direkten Eigennutzen aber mit potentiellem Nutzen für die Gruppe gleichartig erkrankter Kinder geforscht werden, acht Projekte $(11,0 \%)$ sollten an gesunden Kindern oder Jugendlichen oder an erkrankten Minderjährigen ohne Bezug zu ihrer Krankheit durchgeführt werden, und 15 Studien $(20,5 \%)$ sollten Anteile von sowohl engem als auch erweitertem Gruppennutzen beinhalten. Hier handelte es sich in der Regel um gruppennützige Studien, in denen neben der Gruppe erkrankter Kinder und Jugendlicher gesunde Probanden als Kontrollgruppe fungieren sollten. 


\section{Nutzen von Studienprojekten}

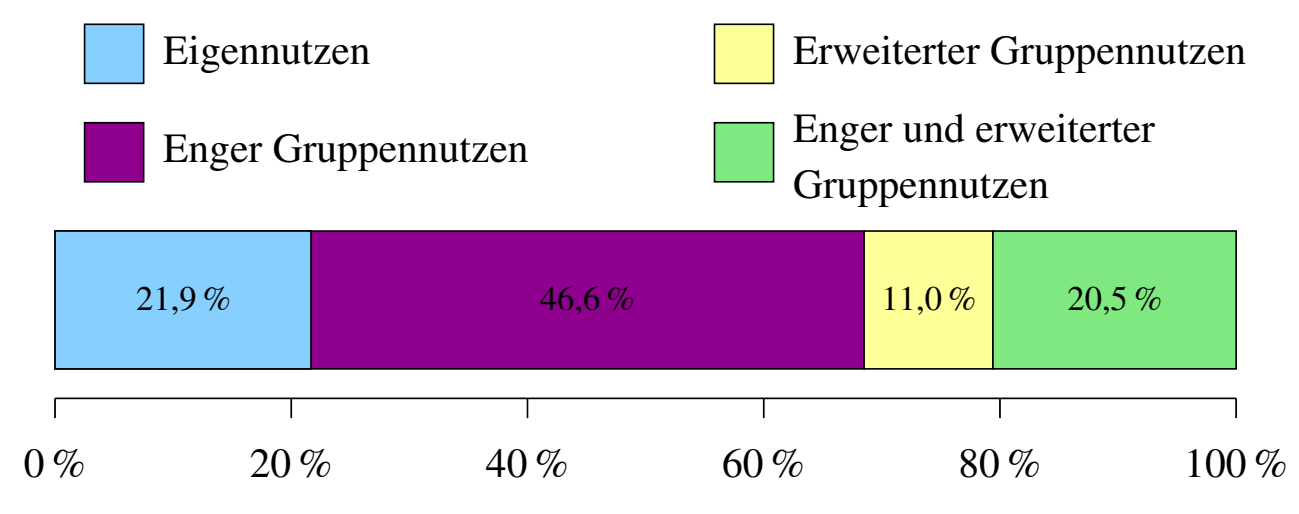

Anteil der Anträge $(\mathrm{N}=73)$

Abbildung 4.2: Anträge zur Forschung an Kindern und Jugendlichen an die Ethikkommission der Universität Göttingen 1999-2003: Nutzen in Kategorien

In sechs $(10,5 \%)$ der 57 geplanten Studienprojekte mit engem oder erweitertem Gruppennutzen oder Anteilen beider Kategorien von Gruppennutzen sollte ein Placebo eingesetzt werden.

\subsubsection{Risiko und Belastung der gruppennützigen Studienprojekte}

Abbildung 4.3 zeigt, dass 19 der gruppennützigen Anträge zur Forschung an Kindern und Jugendlichen $(33,3 \%)$ Eingriffe enthielten, die mit mehr als minimalem Risiko behaftet waren. 18 Anträge $(31,6 \%)$ enthielten Eingriffe mit mehr als minimaler Belastung. Sieben Anträge (9,6\%) enthielten zwei und ein Antrag $(1,4 \%)$ drei verschiedene Interventionstypen mit mehr als minimalem Risiko und vier Anträge $(5,5 \%)$ zwei verschiedene Interventionstypen mit mehr als minimaler Belastung. In 18 Fällen handelte es sich dabei um Venenpunktionen, die nur zu Forschungszwecken nötig wurden und in sechs Fällen um die Gabe eines Placebos. In einem Fall sollte das Placebopräparat intramuskulär injiziert werden. Weiterhin waren in einem Fall eine intravenöse Kontrastmittelgabe und in zwei Fällen die Entnahme einer Gewebebiopsie geplant. Geplante Eingriffe mit nur minimalem Risiko und evtl. minimaler Belastung waren hingegen körperliche Untersuchungen (14-mal), die Erhebung psychometrischer (13-mal) und morphometrischer (siebenmal) Daten, weitere nicht-invasive Untersuchungen wie Sonographien (neunmal) und geringfügige zusätzliche Blutentnahmen ohne die Notwendigkeit einer zusätzlichen Punktion (achtmal) u. a. Eine detaillierte Auflistung aller zu Studienzwecken geplanten Interventionen wird in Abschnitt 8.1 gezeigt. 


\begin{abstract}
Risiko und Belastung in Kategorien minimal mehr als minimal

Risiko

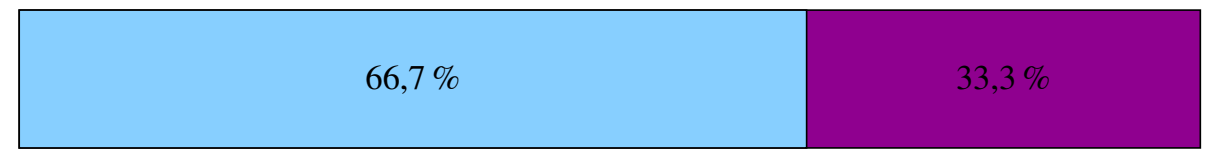

Belastung
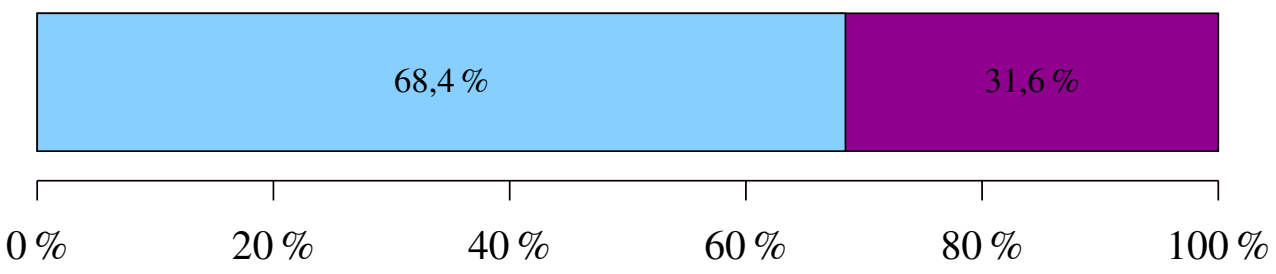

Anteil der Anträge $(\mathrm{N}=57)$

Abbildung 4.3: Anträge zu gruppennütziger Forschung an Kindern und Jugendlichen an die Ethikkommission der Universität Göttingen 1999-2003: Risiko bzw. Belastung in Kategorien
\end{abstract}

\title{
4.2.3 Studien mit Arzneimitteln oder Medizinprodukten
}

Abbildung 4.4 zeigt, dass bei insgesamt 36 der ausgewerteten geplanten Studien (34,2\% bzw. 15,1\%) mit Arzneimitteln oder Medizinprodukten geforscht werden sollte.

\subsubsection{Aufwandsentschädigung}

Abbildung 4.5 zeigt den Anteil geplanter Studien zur Forschung an Kindern und Jugendlichen, die die Zahlung einer Aufwandsentschädigung vorsahen. In zehn der ausgewerteten Anträge $(13,7 \%)$ war eine solche Honorierung der Studienteilnahme geplant.

\subsubsection{Zusammenfassung}

Mit einem Anteil von 6,6\% unter allen Anträgen in den Jahren 1999 bis 2003 spielen Anträge zur Forschung an Kindern und Jugendlichen an die Göttinger Ethikkommission eine nicht unbedeutende Rolle.

Nur gut ein Fünftel $(21,9 \%)$ aller ausgewerteten Anträge stellt geplante Studien dar, die ausschließlich einen direkten Eigennutzen für den minderjährigen Studienteilnehmer beabsichtigen. Knapp die Hälfte (42,6\%) der Anträge betreffen Studien mit engem Grup- 
Studien mit Arzneimitteln/Medizinprodukten

$\square$ Studien mit

Arzneimitteln

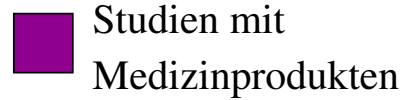

Medizinprodukten

\section{Weder noch}

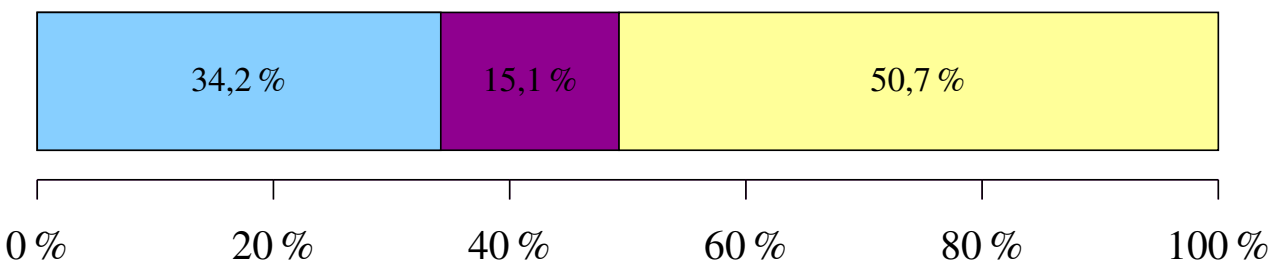

Anteil der Anträge (N=73)

Abbildung 4.4: Anträge zur Forschung an Kindern und Jugendlichen an die Ethikkommission der Universität Göttingen 1999-2003: Handelt es sich um Studien mit Arzneimitteln oder Medizinprodukten?

Aufwandsentschädigung
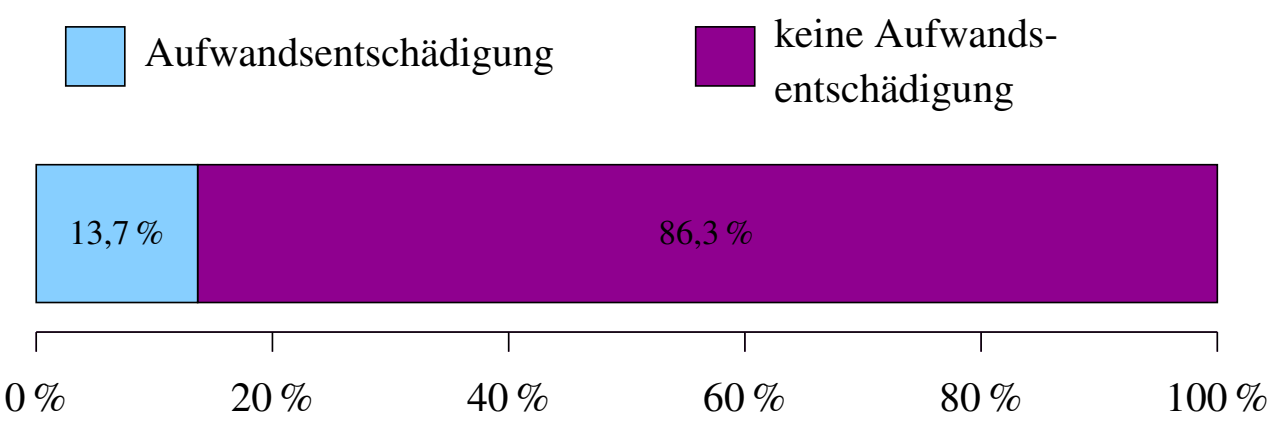

Anteil der Anträge (N=73)

Abbildung 4.5: Anträge zur Forschung an Kindern und Jugendlichen an die Ethikkommission der Universität Göttingen 1999-2003: Ist eine Aufwandsentschädigung für die Versuchspersonen vorgesehen? 
pennutzen. Studien mit erweitertem Gruppennutzen (11\%) und Anteilen beider Kategorien von Gruppennutzen (20,5\%) sind ebenfalls keine Besonderheit. Auch Placeboforschung kommt mit einem Anteil von 10,5\% an allen gruppennützigen Forschungsprojekten nicht selten vor.

Risiko und Belastung sind in 33,3\% bzw. 31,6\% der gruppennützigen Anträge mehr als minimal. Die Ursache dafür sind überwiegend venöse Punktionen ausschließlich zu Forschungszwecken und Forschung mit Einsatz eines Placebos, aber auch invasivere Eingriffe, wie intramuskuläre Injektionen und Gewebebiopsien, kommen vor.

Nur 49,3\% der Anträge beschreiben Studien mit Arzneimitteln oder Medizinprodukten. $50,7 \%$ der Studienprojekte waren damit von keiner gesetzlichen Regelung erfasst.

Die Honorierung einer Studienteilnahme ist mit einem Anteil von 13,6\% in allen ausgewerteten Anträgen eher die Ausnahme als die Regel. 


\section{Vignettenstudie}

\subsection{Methodik}

\subsubsection{Vignettenstudien}

Um die Bedeutung des minimalen Risikos und der minimalen Belastung für die Beurteilung gruppennütziger Studien an Kindern und Jugendlichen zu untersuchen, wurden die Vorsitzenden der deutschen Ethikkommissionen befragt. Diese Befragung hatte weiterhin zum Ziel, die Einstellung erfahrener Mitglieder von Ethikkommissionen zur Beteiligung des Minderjährigen an Entscheidungen über seine Versuchsteilnahme zu eruieren. Sie fand in der Form einer Vignettenstudie statt, die methodisch an die 1951 von Peter H. Rossi in den USA entwickelte Methodik der Vignettenanalyse (Rossi 1979) angelehnt ist.

Vignettenanalysen, auch factorial surveys genannt, erlauben die Entschlüsselung normativer Strukturen bei der Bewertung von Situationen mit komplexen Merkmalskonstellationen (Beck und Opp 2001). Die Einstellungen und Urteile einer bestimmten Personengruppe werden kontext- und situationsgebunden abgefragt, indem ein beispielhafter Fall oder eine durch unterschiedliche Merkmale geprägte Situation prägnant in standardisierter Form, als Vignette, schriftlich charakterisiert wird und der Befragte anschließend sein Urteil dazu abgibt (Friedrichs 1974, Rossi 1979).

Vignettenstudien bieten die Möglichkeit, Bewertungsmomente realitätsnah zu rekonstruieren, indem Fallbeispiele auf die Sicht eines bestimmten Personenkreises, in diesem Fall die Vorsitzenden von Ethikkommissionen, zugeschnitten werden. Durch die Standardisierung der Vignetten ergibt sich aus den Ergebnissen ein Einblick in die Einstellungen und das Entscheidungsverhalten einer Gruppe sowie deren Urteile und Begründungen. Vignettenstudien sind wirklichkeitsnah und bieten gerade in der manchmal eher theoriegeprägten Diskussion um medizinethische Problemfälle einen realistischen Ausgangspunkt. Dabei werden die Vorteile von Vignettenstudien mit den allgemeinen Vorteilen einer schriftlichen Befragung kombiniert. Die Befragten haben beispielsweise die Möglichkeit, Fragen in Ruhe zu durchdenken, und Antworten werden, im Gegensatz zur Situation eines Interviews, nicht durch Merkmale und Verhalten von Interviewern beeinflusst (Diekmann 2002). 
Hindernisse stehen einer Rekonstruktion der Wirklichkeit insofern im Weg, als dass die in einer Vignette vorgestellten Fälle in ihrer Knappheit nur einen Ausschnitt tatsächlich vorkommender Probleme darstellen. Sie sind schriftlich dargestellt, und der Befragte hat keine Möglichkeit zur Rückfrage bei Verständnisschwierigkeiten (Diekmann 2002). So können Detailaspekte subjektiv interpretiert und Antworten dadurch beeinflusst werden. Allgemein gültige Aussagen können daher nicht ausschließlich basierend auf den Ergebnissen einer Vignettenstudie getroffen werden.

Vignettenstudien wurden bisher in Deutschland beispielsweise durchgeführt, um die Einstellungen von Ärzten und Pflegepersonal zum Behandlungsverzicht bei Schwerstkranken (Schöne-Seifert und Eickhoff 1996) zu untersuchen. Weltweit und besonders in den USA haben sie große Bedeutung und wurden mehrfach in der Forschung zu soziologischen und medizinethischen Fragestellungen eingesetzt (Aberegg et al. 2005, Hoffmaster et al. 1991, Roberts et al. 2005, Wertz und Fletcher 1989).

\subsubsection{Konzeption des Fragebogens}

Zur Beantwortung der in Abschnitt 4 und Abschnitt 5 beschriebenen Fragestellungen wurde ein Fragebogen konzipiert, der an die Vorsitzenden der deutschen Ethikkommissionen als in der Regel in Forschungsfragen erfahrene Personen und wichtige Entscheidungsträger innerhalb einer Kommission versandt wurde.

Per Post wurde der Fragebogen mit einem Anschreiben und einem frankierten Rückumschlag zur Bearbeitung (geschätzte Zeit: 20 Minuten) an die im Sommer 2001 durch Internetrecherche ermittelten 49 Vorsitzenden der Ethikkommissionen der deutschen Universitäten und Ärztekammern sowie an die Vorsitzenden freier Ethikkommissionen wie der Ethikkommission International Freiburg und der Freien Ethikkommission Grünstadt geschickt. Zwei Monaten nach dem Versand der Fragebögen erhielten alle Vorsitzenden ein Erinnerungsschreiben. 59,2\% der Vorsitzenden sandten, meist anonym, einen auswertbaren Fragebogen zurück (siehe Abschnitt 5.2).

Im Fragebogen wurden zunächst einige persönliche Daten der Vorsitzenden erhoben (siehe Abschnitt 5.1.2.1).

Anschließend wurden sechs Fallvignetten zur Forschung an Minderjährigen unterschiedlicher Altersstufen vorgestellt, die nach einem einheitlichen Schema aufgebaut waren, sich aber u. a. in ihrem Grad von Risiken und Belastungen unterschieden (siehe Abschnitt 5.1.2.2) und so verschiedene Konstellationen von Forschung ohne direkten potentiellen Nutzen an Kindern und Jugendlichen illustrierten. Die Vorsitzenden wurden im Anschluss gefragt, ob sie das vorgestellte Studienprojekt für zulässig hielten. Zur Beantwortung dieser Frage wurden ihnen unterschiedliche Entscheidungsalternativen angeboten (siehe Abschnitt 5.1.2.3). 
Zusätzlich wurden die Vorsitzenden um freie Anmerkungen zu den Fallbeispielen und um eine Begründung ihrer Entscheidung gebeten. So bot sich die Möglichkeit, einen Einblick in individuelle Überlegungen zu der jeweiligen Vignette und in die Begründungen der Vorsitzenden ihrer Entscheidung zu gewinnen.

Abschließend folgte ein Komplex aus drei weiteren Fragen zur Bedeutung der Beteiligung des Minderjährigen an Entscheidungen über seine Versuchsteilnahme (siehe Abschnitt 5.1.2.3)

Im Folgenden werden die einzelnen Abschnitte des Fragebogens detailliert beschrieben.

\subsubsection{Charakteristika der Vorsitzenden}

Die Vorsitzenden wurden gebeten, Angaben zu ihrem Geschlecht, ihrem Alter, ihrem erlernten Beruf bzw. ihrer Funktion in der Ethikkommission ${ }^{23}$, zu ihrer Erfahrung in der Mitarbeit in Ethikkommissionen sowie ggf. zu Anzahl und Alter eigener Kinder zu machen.

\subsubsection{Fallvignetten}

Sämtliche Vignetten enthielten Anteile von Forschung ohne direkten potentiellen Nutzen für den Versuchsteilnehmer, jedoch mit Aussicht auf einen Nutzen für Kinder und Jugendliche mit der gleichen Erkrankung (enger Gruppennutzen) oder für andere Kinder und Jugendliche (erweiterter Gruppennutzen). In zwei Forschungsprojekten sollte ein Placebopräparat zum Einsatz kommen. Die Vignetten unterschieden sich außerdem im Grad der Risiken und Belastungen und im Alter der betroffenen Kinder. ${ }^{24}$

Die Vignetten orientierten sich an realen Forschungsszenarien in der Kinderheilkunde und Jugendmedizin und in der Kinder- und Jugendpsychiatrie.

Insgesamt wurden je sieben bis zehn Merkmale zur Charakterisierung des Forschungsprojektes genannt:

${ }^{23}$ Folgende Berufe waren vorgegeben: Pädiater/-in, Rechtsmediziner/-in, Pharmakologe/-in, andere ärztliche Tätigkeit, Medizinische/-r Biometriker/-in und Statistiker/-in, Pharmazeut/-in, Jurist/-in, Theologe/in, Philosoph/-in, Medizinethiker/-in, Student/-in, Pflegevertreter/-in, Bürgervertreter/-in (Laie) und Sonstiges. Mehrfachnennungen waren möglich.

${ }^{24}$ Der Pädiater Hannsjörg W. Seyberth unterscheidet fünf unterschiedliche Entwicklungsstadien eines Kindes: Das Frühgeborene im Stadium des reinen Überlebens, das Neugeborene im Stadium der Anpassung nach der Geburt, das Kleinkind im Stadium der Proliferation und des Wachstums, das Schulkind im Stadium der Differenzierung und des Trainierens und der Adoleszent im Stadium des Gewinns der Reproduktionsfähigkeit und der endgültigen Körpergröße (Seyberth 2005). An vier dieser Entwicklungsphasen (Neugeborenes, Kleinkind, Schulkind und Adoleszent) orientierten sich die Vignetten. 
- Eine kurze Beschreibung des Krankheitsbildes, zu dem geforscht werden sollte,

- die Art des geplanten Projektes,

- sein zeitlicher Umfang,

- die Gruppe, der die Forschung potentiell nützen sollte,

- ggf. der Einsatz eines Placebopräparates,

- ggf. die Anzahl der Interventionen,

- ggf. die Anzahl der zu entnehmenden Proben,

- das Alter des Kindes,

- die voraussichtliche Anzahl der Patienten/Probanden und

- der wissenschaftliche Zweck der Forschung.

Im Folgenden werden die Originalvignetten aufgeführt und ihre Charakteristika beschrieben (Originalfragebogen siehe Abschnitt 8.2).

\section{Vignette 1: Venöse Blutentnahme ohne Notwendigkeit zusätzlicher Punktion bei Rett-Syndrom}

„Das Rett-Syndrom ist eine Erkrankung mit verzögerter geistiger Entwicklung, Autismus, Epilepsie und weiteren neurologischen Auffälligkeiten, die sich im Säuglingsalter manifestiert und fast ausschließlich bei Mädchen vorkommt. Als Ursache konnte eine Genmutation (MECP2) nachgewiesen werden. Aber auch bei Mädchen und Jungen mit unspezifischer verzögerter geistiger Entwicklung konnte eine Mutation im MECP2-Gen nachgewiesen werden. Um das gesamte klinische Spektrum dieser Genmutation zu erforschen, soll das Blut von Patienten mit mentaler Retardierung unklarer Genese genetisch analysiert werden. Geplant ist eine Untersuchung von acht Gruppen mit je 15 Patienten im Alter von zwei bis fünf Jahren. Für die Untersuchung werden pro Patient fünf ml Blut benötigt, die im Rahmen ohnehin notwendiger Blutentnahmen entnommen und anonymisiert ausgewertet werden sollen."

Bei dieser Vignette handelt es sich nicht um Forschung nach dem AMG oder MPG.

Das Forschungsprojekt ist ein Beispiel für Forschung mit potentiellem Nutzen für die Gruppe der Kinder mit gleicher Erkrankung und daher für enge Gruppennützigkeit.

Bei dieser Vignette wird ein Eingriff vorgeschlagen, der in der nationalen und internationalen Literatur oft als Beispiel für Forschung mit minimalem Risiko genannt wird (British Paediatric Association 1980, Stellungnahme der ZEKO 2004): Im Rahmen einer sowieso notwendigen Blutentnahme wird aus einer Vene eine zusätzliche Blutprobe entnommen. Das bedeutet für das betroffene Kind keinen zusätzlichen Schmerz, sondern, wenn man 
die Ausführung durch eine geübte Person voraussetzt ${ }^{25}$, nur eine Verlängerung der Prozedur um einige Sekunden. Das geringe, bei jeder Blutentnahme vorhandene Risiko von Infektion, Nervenschädigung und Hämatom fällt nicht gesondert ins Gewicht, weil die Blutentnahme in jedem Fall erforderlich ist. Die psychische Belastung durch Angst vor der Nadel und dem Schmerz kann bei einem nicht an Blutentnahmen gewöhnten Kind erheblich sein. Sie wird sich aber durch eine um kurze Zeit verlängerte Dauer des Eingriffs kaum vergrößern. Daher kann nicht nur das Risiko, sondern auch die Belastung durch die Studienteilnahme als minimal kategorisiert werden. ${ }^{26}$

Die Versuchspersonen dieser Studie sind zwei bis fünf Jahre alt. Sie sind in einem Alter, in dem sie durchaus bewusst wahrnehmen, dass ein medizinischer Eingriff an ihnen vorgenommen werden soll. Einfache Erklärungen können ihnen helfen, ein gewisses Verständnis für das, was mit ihnen geschieht, zu entwickeln. ${ }^{27}$ Hier handelt es sich allerdings um mental retardierte Kinder, deren intellektuelle Entwicklung nicht dem Durchschnitt ihrer Altersgenossen entspricht.

\section{Vignette 2: Psychokognitive Tests bei HKS und LRS}

„Im Rahmen einer Studie soll das überzufällig häufige gemeinsame Auftreten von Hyperkinetischer Störung (HKS) und Lese-Rechtschreib-Schwäche (LRS) untersucht werden. Bei der beantragten Studie sollen verschiedene Ursachen beider Störungen analysiert werden. Ziel ist es, die krankheitsauslösenden Ursachen zu erklären, um eine genaue und frühzeitige Diagnostik und Therapie zu ermöglichen. Hierfür werden mindestens 80 Kinder mit Lese-Rechtschreib-Schwäche und/oder Hyperkinetischer Störung zwischen sechs und elf Jahren sowie zum Vergleich eine Zahl gesunder Kinder untersucht. Die Testdauer

\footnotetext{
${ }^{25}$ Wenn es um die Ausführung medizinischer Eingriffe geht, wird im Folgenden immer davon ausgegangen, dass die ausführende Person optimal darin geschult ist und lange Erfahrung in dieser Ausführung besitzt, den Eingriff also bestmöglich ausführen kann.

${ }^{26}$ Diskutieren kann man im Zusammenhang mit der Studie noch den Aspekt, dass es sich bei den Probanden um mental retardierte, besonders schutzbedürftige Kinder handelt. Das Studiendesign ähnelt dem 2001 von Dörner und Spielmann beschriebenen „Eisingen-Fall“ (Dörner und Spielmann 2001). Hier hatten in den Jahren 1994 und 1995 Mitarbeiter des Instituts für Humangenetik der Universität Würzburg Blutproben von 179 geistig behinderten, in einem katholischen Heim lebenden Kindern für genetische Untersuchungen zum Fragilen-X-Syndrom verwendet, ohne für adäquate Information der Kinder, Eltern oder der legalen Betreuer zu sorgen. $80 \%$ der entnommenen Blutproben waren zusätzlich im Rahmen einer sowieso zu tätigenden freiwilligen Blutentnahme entnommen wurden. Die Kritik an diesem Vorgehen fokussierte nicht nur das Fehlen des informed consent, sondern auch die grundsätzliche moralische Zweifelhaftigkeit des Vorgehens, mental retardierte Kinder zu nicht eigennützigen Versuchszwecken heranzuziehen. Wenn man die vorgestellte Vignette mit dem „Eisingen-Fall“" vergleicht, muss betont werden, dass die beiden sich in einem wesentlichen Punkt unterscheiden: Im „Eisingen-Fall“ ging es um in einer Anstalt untergebrachte und damit besonders vulnerable Kinder. Diese Bedingung ist bei der oben geschilderten Vignette nicht gegeben.

${ }^{27}$ Bei allen hier vorgestellten Vignetten ist davon auszugehen, dass darüber hinaus gehende Standards der Ethik in der klinischen Forschung am Menschen (informed consent, Anonymisierung von Daten und entnommenen Körpermaterialien etc.) gewahrt werden.
} 
beträgt insgesamt sieben bis acht Stunden inklusive Pausen, verteilt auf drei Tage innerhalb einer Woche. Zu den Untersuchungen gehören: Neurologische Untersuchung, Hörtest, Fragebögen, etablierter Intelligenztest für das Kindesalter, Lese-Rechtschreib-Tests, Aufmerksamkeitstests. Bei einer Sitzung wird ein Elektroenzephalogramm (EEG) abgeleitet.“

Bei dieser Vignette handelt es sich nicht um Forschung nach dem AMG oder MPG.

Das Forschungsprojekt ist einerseits ein Beispiel für Forschung mit potentiellem Nutzen für die Gruppe der Kinder gleichen Alters mit gesunden Kindern als Versuchspersonen und daher für erweiterte Gruppennützigkeit. Andererseits ist es auch ein Beispiel für Forschung mit potentiellem Nutzen für die Gruppe der Kinder mit gleicher Erkrankung mit kranken Kindern als Versuchspersonen und somit für enge Gruppennützigkeit.

Die Tests sind Untersuchungen, bei denen den Kindern kein physischer Schmerz zugefügt wird. Eine ausführliche neurologische Untersuchung dauert etwa eine halbe Stunde. Das Kind muss sich dafür ausziehen, verschiedene Aufgaben ausführen und in Kontakt mit dem Untersucher treten. Das Ableiten eines EEGs ist nicht schmerzhaft. Mehrere Elektroden werden auf dem Kopf des Kindes befestigt, mit Kabeln verbunden und dort für etwa eine halbe Stunde belassen. Für Hörtest, Fragebögen, Intelligenz-, Lese-Rechtschreibund Aufmerksamkeitstests muss sich das Kind für eine gewisse Zeit konzentrieren. Die verschiedenen Tests könnten bei Kindern Stress auslösen, gerade wenn sie dazu führen, dass sich Kinder untereinander vergleichen, oder wenn bei einzelnen das Gefühl entsteht, Anforderungen nicht erfüllen zu können. Deswegen muss bei den Tests besonderer Wert auf eine spielerische, stressfreie Atmosphäre, ohne die Verletzung von Schamgefühlen, gelegt werden. Das Risiko dieser Studie ist minimal, die Belastung kann unter den im vorigen Satz genannten Bedingungen ebenfalls als minimal kategorisiert werden.

Die Kinder in der Vignette sind sechs bis elf Jahre alt. Über diese Altersgruppe wird hinsichtlich der Bedeutung ihrer Einstellung an Entscheidungen über eine Versuchsteilnahme kontrovers diskutiert (siehe Abschnitt 2.3.3). In diesem Alter können Kinder in der Regel lesen und schreiben, trotzdem liegen ihre Fähigkeiten im Verständnis komplexer Zusammenhänge noch weit hinter denen von Jugendlichen und Erwachsenen.

\section{Vignette 3: Orale Placebogabe mit Verzicht auf Standardtherapie bei allergischem Schnupfen}

„Im Rahmen einer randomisierten, placebokontrollierten, multizentrischen Phase-2-Doppelblindstudie erhalten Allergiker mit ganzjährig auftretendem allergischem Schnupfen (Hausstaubmilbe) ab einem Alter von zwölf Jahren einmal täglich eine Tablette eines neu entwickelten Antihistaminikums über einen Zeitraum von sechs Monaten. Das Medikament wird gegen Placebo getestet, da die bisherige medikamentöse Behandlung unzuverlässig ist und keine vollständige Symptomfreiheit bringt. Die allergischen Symptome werden in einem Tagebuch dokumentiert.“ 
Die Vignette beschreibt eine Studie nach dem deutschen AMG.

Das Forschungsprojekt ist ein Beispiel für Forschung mit potentiellem Nutzen für die Gruppe der Kinder und Jugendlichen mit gleicher Erkrankung und somit für enge Gruppennützigkeit.

Es handelt sich um Kinder und Jugendliche, die an allergischem Schnupfen leiden. Symptome einer allergischen Rhinitis sind Niesreiz, Fließschnupfen, Schwellung der Nasenschleimhaut und fakultativ Entzündungen der Nase und der Nasennebenhöhlen (Rassner 2002). Diese Beschwerden sind nicht lebensbedrohlich, aber lästig und beeinträchtigen die Allergiker in ihrem Alltag. Ein neues antiallergisches Mittel soll im Rahmen einer Phase-2-Studie an ihnen erprobt werden. ${ }^{28}$ Da die etablierte Therapie (Cromoglicinsäure, lokal und systemisch angewendete Antihistaminika und Schleimhautkortikoide (Rassner 2002)) gegen den durch Hausstaubmilben verursachten allergischen Schnupfen nicht zuverlässig ist, wird dieses neue Medikament gegen Placebo getestet. Der Versuch soll über einen Zeitraum von einem halben Jahr stattfinden: Die Studiengruppe erhält einmal täglich eine Tablette des neues Antihistaminikums, während die andere Gruppe ein Placebo bekommt. In die beiden Gruppen werden die Patienten durch ein Randomisierungsverfahren eingeteilt. Die Studie ist eine Doppelblindstudie: Weder Forscher noch Versuchsperson wissen, wer das neue Medikament und wer das Placebopräparat erhält. Zum Vergleich der beiden Gruppen sollen die Kinder Tagebuch über das Auftreten ihrer allergischen Symptome führen. Die Patienten der Studiengruppe erhalten Tabletten des neuen Antihistaminikums und können ggf. von den Chancen einer neuen Behandlung profitieren. Die Kontrollgruppe erhält ein Placebopräparat und wird damit für ein halbes Jahr nicht effektiv behandelt. Diese Patienten sind ggf. ihren Allergiesymptomen ausgesetzt. Die Vignette thematisiert u. a. die Frage, ob Placeboforschung bei Kindern und Jugendlichen erlaubt sein soll und ob bei einer Erkrankung mit geringfügigen Symptomen und unzuverlässiger Standardtherapie zugunsten eines Placebopräparates auf diese Standardtherapie verzichtet werden kann. Das Risiko und die Belastung dieser Studie sind als größer als minimal zu kategorisieren.

Die Kinder bzw. Jugendlichen sind mindestens zwölf Jahre alt. Sie können ihre Krankheit verstehen und sind möglicherweise gut darüber informiert. In der Regel sind sie kompetent, den Sinn der klinischen Studie zu begreifen.

\section{Vignette 4: Herzmuskelbiopsie bei ohnehin notwendiger Herzoperation}

„,20 Säuglinge im Alter zwischen zwei Wochen bis drei Monaten mit angeborenen Herzfehlern und klinisch manifester Herzinsuffizienz nehmen präoperativ an einer Medikamentenstudie teil. In einer randomisierten Medikamentenstudie erhalten die Kinder der

\footnotetext{
${ }^{28}$ In Phase-2-Studien geht es um die Wirksamkeit eines Medikaments, die Ermittlung seines Dosisbereichs und um häufige Nebenwirkungen. Die Studien werden an 100-300 ausgewählten Patienten durchgeführt (siehe auch Abschnitt 2.1).
} 
Studiengruppe (zehn Patienten) neben der Standardtherapie (Digoxin, Diuretikum) zusätzlich den Betarezeptorenblocker Propranolol, während die Kinder der Kontrollgruppe (zehn Patienten) ausschließlich mit der Standardtherapie behandelt werden. Im Rahmen der Herzoperation wird bei allen Kindern eine Herzmuskelbiopsie für rein wissenschaftliche Zwecke entnommen."

Die Vignette beschreibt eine Studie mit zwei Teilaspekten. Es handelt sich um ein Beispiel für Forschung mit potentiellem Nutzen für die Gruppe der Kinder und Jugendlichen mit gleicher Erkrankung und somit für enge Gruppennützigkeit. Die Testung des Betarezeptorenblockers stellt eine Medikamentenstudie nach dem deutschen AMG dar. Hierbei wird die Kontrollgruppe mit der Standardtherapie behandelt. Durch ein Randomisierungsverfahren werden die Kinder der einen oder anderen Gruppe zugeteilt.

Der zweite Teil der Studien beinhaltet eine Herzmuskelbiopsie im Rahmen einer sowieso notwendigen Operation, deren Risiko als mehr als minimal zu werten ist. Die Belastung der Babys durch diesen Eingriff unter Vollnarkose ist minimal.

Bei den Kindern dieser Vignette handelt es sich um Babys im Alter von zwei Wochen bis drei Monaten. Die Frage, inwieweit die Kinder für oder gegen ihre Studienteilnahme entscheiden können, stellt sich hier darum nicht. Gefordert ist die Entscheidung der Eltern im Sinne des informed consent.

\section{Vignette 5: Intramuskuläre Placeboinjektionen bei angeborenem Herzfehler}

„Kinder mit angeborenen Herzfehlern haben eine erhöhte Erkrankungshäufigkeit für Herzmuskelentzündungen, die durch RS-Viren hervorgerufen werden. Im Rahmen einer randomisierten, placebokontrollierten Studie soll getestet werde, ob eine prophylaktische Anwendung von Immunglobulinen die Erkrankungshäufigkeit für Herzmuskelentzündungen senkt. Kinder im Alter zwischen zwei und fünf Jahren und mit angeborenem Herzfehler erhalten prophylaktisch fünf Immunglobulin-Injektionen intramuskulär. Den Kindern der Kontrollgruppe werden fünf Injektionen physiologischer Kochsalzlösung gegeben. Im Verlauf wird beobachtet, wie viele Kinder von jeder Gruppe an Myokarditis erkranken. Die Erkrankungsfälle werden mit der Standardtherapie behandelt.“

Die Vignette beschreibt eine Studie nach dem deutschen AMG.

Das Forschungsprojekt ist ein Beispiel für Forschung mit potentiellem Nutzen für die Gruppe der Kinder mit gleicher Erkrankung und somit für enge Gruppennützigkeit.

Im Rahmen einer multizentrischen Studie werden mit einem Randomisierungsverfahren Kinder mit angeborenen Herzfehlern zwei Gruppen zugeteilt. Die Studiengruppe erhält fünf Injektionen mit Immunglobulinen intramuskulär, während die Kontrollgruppe fünf Injektionen eines Placebos (physiologische Kochsalzlösung) bekommt. Intramuskuläre 
Injektionen werden bei Kindern üblicherweise in die Gluteal- oder Oberschenkelmuskulatur verabreicht. Die Risiken intramuskulärer Injektionen sind eine Blutung und die Entstehung eines Hämatoms im Muskel, insbesondere bei Gerinnungsstörungen, außerdem die Verletzung von Nerven sowie Infektionen und Abszesse. Wird nach Einführen der Nadel die Punktion eines Blutgefäßes nicht korrekt durch Aspiration ausgeschlossen, kann die Injektionslösung intravasal injiziert werden. Die Kinder der Studiengruppe sind den unerwünschten Wirkungen der Immunglobuline, insbesondere Überempfindlichkeitsreaktionen (Resch und Szamel 2001), ausgesetzt. Gleichzeitig ist ihr Risiko, an einer Entzündung des Herzmuskels zu erkranken, ggf. niedriger als bei den nicht behandelten Kindern der Kontrollgruppe, da sie eine neue potentiell wirksame Prophylaxe erhalten. Die Kinder der Kontrollgruppe erhalten fünf Placeboinjektionen, die dasselbe Risiko von Komplikationen einer intramuskulären Injektionen beinhalten. Injektionen können für Kinder sehr belastend sein. Viele fürchten den Injektionsvorgang und damit verbundene Schmerzen. Die Injektionen und die mit der Studie verbundenen Kontrolluntersuchungen verpflichten die Kinder und ihre Eltern oder Betreuer zu regelmäßigen Arztbesuchen. Risiko und Belastung dieser Studie sind mehr als minimal.

Die Versuchspersonen in dieser Studie sind zwei bis fünf Jahre alt. Sie sind damit in einem Alter, in dem sie den medizinischen Eingriff und seine Folgen durchaus bewusst erleben.

\section{Vignette 6: Zusätzliche Knochenmarkpunktionen bei ALL}

„In einer randomisierten therapeutischen Medikamentenstudie wird bei Kindern (sechs bis elf Jahre alt) mit akuter lymphatischer Leukämie (ALL) ein neues Chemotherapieschema gegen die etablierte Therapie getestet. Im Studienverlauf werden bei beiden Gruppen im wöchentlichen Abstand Knochenmarkpunktionen vorgenommen. Die Kinder erhalten hierfür ein leichtes Schlafmittel und eine lokale Betäubung der Haut (Emla-Pflaster). Die Knochenmarkpunktionen erfolgen teilweise zur Verlaufskontrolle, teilweise zu reinen Studienzwecken. Insgesamt werden sechs von zehn Knochenmarkpunktionen ausschließlich zu wissenschaftlichen Zwecken durchgeführt. Hierbei sollen minimale molekulare Veränderungen (minimal residual disease) untersucht werden.“

Die Vignette beschreibt eine Studie nach dem deutschen AMG.

Das auf die molekularen Veränderungen im Knochenmark abzielende Forschungsprojekt ist ein Beispiel für Forschung mit potentiellem Nutzen für die Gruppe der Kinder mit gleicher Erkrankung und damit für enge Gruppennützigkeit.

Kinder, die an akuter lymphatischer Leukämie leiden, werden nach einem neuen Chemotherapieschema behandelt. Im Rahmen dieser Behandlung soll das Krankheitsbild der Kinder weiter erforscht werden. Die Kinder sollen sich wöchentlichen Knochenmarkpunktionen unterziehen. Durch die Gabe eines leichten Schlafmittels und die Betäubung der Haut mit einem Pflaster sollen die Punktionen für die Kinder so wenig belastend und 
schmerzhaft wie möglich gestaltet werden. Die Knochenmarkpunktion wird z. B. am hinteren Beckenkamm durchgeführt. Nach Desinfektion und örtlicher Betäubung wird mit dem Skalpell eine drei bis vier Millimeter große Hautinzision gesetzt, eine Kanüle eingeführt und ins Knocheninnere gebracht. So kann Knochenmark aspiriert und eine Knochenmarkstanze entnommen werden. Insbesondere die Aspiration ist schmerzhaft. Nach der Punktion muss das Kind einige Zeit still liegen. Die Risiken von Knochenmarkpunktionen sind Blutung, Infektion, Nervenverletzung und Hämatom. Die psychische Belastung durch Knochenmarkpunktionen kann beträchtlich sein. Der Eingriff ist invasiv und trotz aller Vorkehrungen schmerzhaft oder zumindest unangenehm. Die Vignette thematisiert Forschung mit mehr als minimalem Risiko und mehr als minimaler Belastung. Allerdings kann man berücksichtigen, dass Kinder mit akuter lymphatischer Leukämie im Rahmen ihrer Behandlung des öfteren Punktionen über sich ergehen lassen müssen. Sie kennen also die Prozedur und wissen, was auf sie zukommt. Dies verringert möglicherweise ihre Angst und das Unbehagen während des Eingriffs. Mit dieser Vignette wird die Frage aufgeworfen, inwiefern Risiko und Belastung in ein Verhältnis zur Grundkrankheit und zu den Erfahrungen der hier genannten sechs- bis elfjährigen Kindern mit bestimmten medizinischen Prozeduren gesetzt werden können.

Die sechs bis elfjährigen Kinder sind in einem Alter, für das in der internationalen Diskussion erwogen wird, die Teilnahme an klinischen Studien vom positiven Votum der Probanden selbst abhängig zu machen (siehe Abschnitt 2.3.3).

\section{Übersicht}

Tabelle 5.1 zeigt eine Übersicht der Charakteristika der Vignetten.

\subsubsection{Fragen zu den Vignetten}

Zur Untersuchung der Frage, ob der Grad von Risiko und Belastung für Vorsitzende deutscher Ethikkommissionen ein Kriterium bei der Beurteilung der Zulässigkeit gruppennütziger Forschung an Kindern und Jugendlichen ist, wurde den Vorsitzenden im Anschluss an die jeweilige Vignette die folgende Frage gestellt:

- Frage 1: „Halten Sie eine solche Studie für vertretbar und würden Sie ein positives Votum einer Ethikkommission befürworten?“”

Vorgegeben waren vier Antwortmöglichkeiten:

1. „Ja, ohne Einschränkungen“,

2. „Ja, mit Einschränkungen (bitte Antwort begründen)“",

3. „Ich weiß nicht/keine Meinung“, 


\begin{tabular}{|c|c|c|c|c|c|c|c|c|c|}
\hline \multirow[t]{2}{*}{$\begin{array}{l}\text { Vig- } \\
\text { nette }\end{array}$} & \multirow[t]{2}{*}{$\begin{array}{l}\text { AMG- } \\
\text { For- } \\
\text { schung }\end{array}$} & \multicolumn{2}{|c|}{$\begin{array}{l}\text { Grup- } \\
\text { pennüt- } \\
\text { zigkeit }\end{array}$} & \multicolumn{2}{|c|}{ Risiko } & \multicolumn{2}{|c|}{$\begin{array}{l}\text { Belas- } \\
\text { tung }\end{array}$} & \multirow[t]{2}{*}{$\begin{array}{l}\text { Alter der } \\
\text { Kinder }\end{array}$} & \multirow[t]{2}{*}{ Besonderheiten } \\
\hline & & $\begin{array}{l}\infty \\
\frac{0}{0}\end{array}$ & 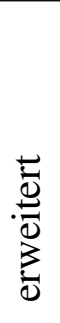 & . & 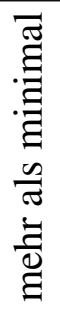 & 点 & 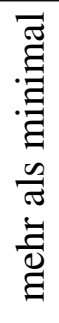 & & \\
\hline 1 & - & + & & + & & + & & 2-5 Jahre & $\begin{array}{l}\text { Mental retardierte } \\
\text { Kinder }\end{array}$ \\
\hline 2 & - & + & + & + & & + & & 6-11 Jahre & - \\
\hline 3 & + & + & & & + & & + & 12-18 Jahre & $\begin{array}{l}\text { Randomisierung und } \\
\text { Einsatz eines } \\
\text { Placebopräparates mit } \\
\text { Verzicht auf } \\
\text { Standardtherapie }\end{array}$ \\
\hline 4 & Anteilig & + & & & + & + & & $\begin{array}{l}2 \text { Wochen- } \\
3 \text { Monate }\end{array}$ & $\begin{array}{l}\text { Randomisierung in } \\
\text { Studien- und } \\
\text { Kontrollgruppe }\end{array}$ \\
\hline 5 & + & + & & & + & & + & 2-5 Jahre & $\begin{array}{l}\text { Randomisierung und } \\
\text { invasive Placebogabe }\end{array}$ \\
\hline 6 & + & + & & & + & & + & 6-11 Jahre & $\begin{array}{l}\text { Knochenmarkpunktio- } \\
\text { nen bei Kindern, die } \\
\text { diese Eingriffe kennen }\end{array}$ \\
\hline
\end{tabular}

Tabelle 5.1: Übersicht der Charakteristika der Vignetten 
4. „Nein, auf gar keinen Fall (bitte Antwort begründen)“.

Anschließend wurde Raum für die Begründung der Entscheidung und sonstige Anmerkungen gelassen.

Um zu ermitteln, welche Bedeutung die Vorsitzenden von Ethikkommissionen der Einstellung eines Kindes zu seiner Studienteilnahme zumessen bzw. ob sie die Zustimmung eines Kindes oder Jugendlichen zu seiner Versuchsteilnahme als notwendige Bedingung für die Akzeptanz einer Studie mit mehr als minimalem Risiko oder mehr als minimaler Belastung ansehen, wurden drei jeweils mit „Ja“ oder „Nein“ zu beantwortende Fragen gestellt:

- Frage 2: „Halten Sie eine individuelle Aufklärung und Zustimmung der Kinder für erforderlich?" 29

- Frage 3: „Wäre ein eindeutig abwehrendes Verhalten des Kindes ein Ausschlussbzw. Abbruchkriterium?“30

- Frage 4: „Halten Sie eine Aufwandsentschädigung für das Kind für notwendig?“

\subsubsection{Auswertung}

Für die Auswertung der quantitativen Daten wurden Häufigkeitszählungen verwendet. ${ }^{31}, 32$

Die frei formulierten Antworten der Vorsitzenden, die Kommentare zu den Vignetten und Begründungen der Entscheidungen enthielten, wurden zum Zwecke der Übersichtlichkeit zu Kategorien gebündelt. Dieser Vorgang erfolgte orientiert am Modell der induktiven Kategorienbildung von Mayring (Mayring 2002). Wenn von einem Vorsitzenden mehrere Anmerkungen zu einem Fall gemacht wurden und unterschiedliche Gründe für eine Entscheidung genannt wurden, so wurden diese als Mehrfachnennungen berücksichtigt und

\footnotetext{
${ }^{29}$ Bei Vignette 4 wurde auf diese Frage verzichtet, da es sich bei den Versuchsteilnehmern um Babys handelte.

${ }^{30}$ Bei Vignette 4 wurde auf diese Frage verzichtet, da es sich bei den Versuchsteilnehmern um Babys handelte.

${ }^{31}$ Die Auswertung dieser Fragen erfolgte prozentual mit Hilfe des Tabellenkalkulationsprogramms OpenOffice.org Calc.

${ }^{32}$ Die Auswertung erfolgte nach Beratung durch die Abteilung Medizinische Statistik der Georg-AugustUniversität Göttingen.
} 
verschiedenen Kategorien zugeordnet. ${ }^{33}, 34$ Anschließend wurde gezählt, wie häufig die einzelnen Kategorien kodiert wurden.

\subsection{Ergebnisse}

Von den 49 angeschriebenen Vorsitzenden reagierten 35 (71,4\%) auf die Anfrage und eine zwei Monate später versandte Nachfrage. Davon sandten 29 Vorsitzende (59,2\%) einen verwertbaren Fragebogen zurück. Sechs Vorsitzende (12,2\%) äußerten, den Fragebogen aus unterschiedlichen Gründen (Zeitmangel, fehlendes Verständnis für die Fragestellung etc.) nicht beantworten zu können.

\subsubsection{Charakteristika der Vorsitzenden}

Tabelle 5.2 zeigt eine Übersicht zu den erhobenen allgemeinen Daten der teilnehmenden Vorsitzenden ${ }^{35}$.

\subsubsection{Zulässigkeit unterschiedlicher Studientypen}

Im Folgenden werden die Ergebnisse zu Frage 1, ,Halten Sie eine solche Studie für vertretbar und würden Sie ein positives Votum einer Ethikkommission befürworten?“ dargestellt. Dabei werden, in der Reihenfolge der Vignetten geordnet, erst die Resultate der quantitativen Auswertung gezeigt und dann die zu Kategorien gebündelten freien Anmerkungen tabellarisch zusammengefasst sowie einige Anmerkungen wörtlich zitiert.

Anschließend werden die Ergebnisse zur Beurteilung der Zulässigkeit der einzelnen in den Vignetten dargestellten Studientypen miteinander verglichen.

\footnotetext{
${ }^{33}$ Man wird also beim Zusammenzählen der in die Kategorien zur jeweiligen Vignette eingegangenen Anmerkungen auf eine von 29 (Anzahl der zurückerhaltenen Fragebögen) abweichende Zahl kommen.

${ }^{34}$ Ein Auswertungsproblem ergab sich vereinzelt, wenn Antwortmöglichkeiten nicht im Konsens mit dem offenen Kommentar waren: So wurde beispielsweise Antwortmöglichkeit 2 „Ja, mit Einschränkungen“ angekreuzt, dann aber bei der offen formulierten Begründung für die Auswahl keine echte Einschränkung angegeben. So stellt die Anmerkung „Schriftliche Aufklärung und Einverständniserklärung der Erziehungsberechtigten ist erforderlich“ (Vorsitzender 11 zu Vignette 1) keine echte Einschränkung dar, da es sich dabei um eine Standardbedingung der klinischen Forschung an Menschen handelt, die als selbstverständlich vorausgesetzt wird. Einige Antworten wurden daher anhand des Begründungstextes gruppiert.

${ }^{35} \mathrm{Im}$ Folgenden wird allerdings, wenn von den Vorsitzenden gesprochen wird, zur sprachlichen Vereinfachung und um die Anonymität der drei weiblichen Vorsitzenden zu gewährleisten, immer die maskuline Form verwendet.
} 
Charakteristika der Vorsitzenden

\begin{tabular}{lll}
\hline Stichprobe (Antwortquote) & & $\mathrm{N}=29 / 49(59,2 \%)$ \\
Alter & $<41$ Jahre & $\mathrm{N}=1(3,4 \%)$ \\
& 41-60 Jahre & $\mathrm{N}=12(41,4 \%)$ \\
& Keine Jahre & $\mathrm{N}=15(51,7 \%)$ \\
& Weiblich & $\mathrm{N}=1(3,4 \%)$ \\
Geschlecht & Männlich & $\mathrm{N}=3(10,3 \%)$ \\
Beruf/Funktion in der & Pädiater & $\mathrm{N}=26(89,7 \%)$ \\
Ethikkommission & Andere ärztliche Tätigkeit & $\mathrm{N}=5(17,2 \%)$ \\
& Pharmakologe & $\mathrm{N}=14(48,3 \%)$ \\
& Pädiater/Pharma- & $\mathrm{N}=5(17,2 \%)$ \\
kologe/Pharmazeut & $\mathrm{N}=1(3,4 \%)$ \\
Jurist & $\mathrm{N}=1(3,4 \%)$ \\
in Ethikkommissionen & Medizinethiker & $\mathrm{N}=3(10,3 \%)$ \\
& $<11$ Sitzungen & $\mathrm{N}=1(3,4 \%)$ \\
Eigene Kinder & $>50$ Sitzungen & $\mathrm{N}=7(24,1 \%)$ \\
& Ja & $\mathrm{N}=21(72,4 \%)$ \\
& Nein & $\mathrm{N}=27(93,1 \%)$ \\
\hline
\end{tabular}

Tabelle 5.2: Charakteristika der Vorsitzenden 


\subsubsection{Vignette 1: Venöse Blutentnahme ohne Notwendigkeit zusätzlicher Punktion bei Rett-Syndrom}

\section{Quantitative Auswertung}

Abbildung 5.1 zeigt, dass die Vorsitzenden Vignette 1 eindeutig zustimmend aufnahmen. Alle 29 Vorsitzenden (100\%) gaben an, sie würden das Projekt befürworten. Von ihnen machte nur ein Vorsitzender einschränkende Bemerkungen zur Versuchsplanung.

Studie vertretbar? Positives Votum?

Ja, ohne Einschränkungen

Ja, mit Einschränkungen

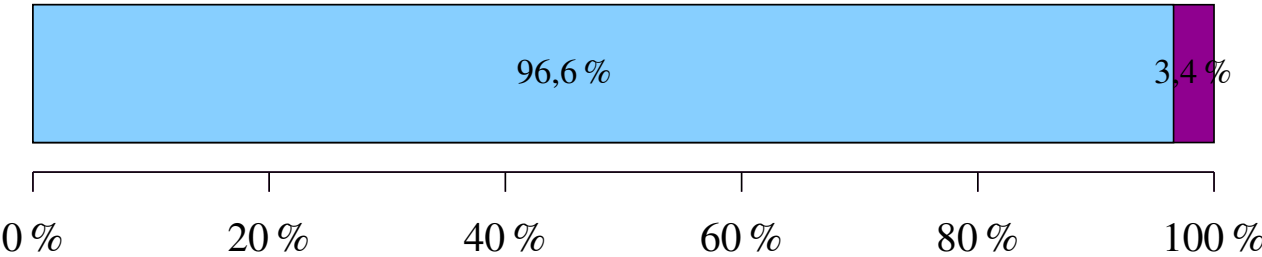

Anteil der Vorsitzenden $(\mathrm{N}=29)$

Abbildung 5.1: Vignette 1, Frage 1: „Halten Sie eine solche Studie für vertretbar und würden Sie ein positives Votum einer Ethikkommission befürworten?“

\section{Auswertung der Anmerkungen}

14 von 29 Vorsitzenden (48,3\%) formulierten Anmerkungen zu dem vorgestellten Fall (siehe Tabelle 5.3).

Keine der Anmerkungen betraf die grundsätzliche Zulässigkeit dieses Typs von Forschung.

Die meisten Vorsitzenden verwiesen mit ihren Anmerkungen auf die Notwendigkeit der Aufklärung (Vorsitzende 5, 6, 11 und 29) und Einwilligung der Eltern (Vorsitzende 11, 13, 14 und 29) bzw. Einwilligung des Kindes,

„wenn dieses das Wesen der Untersuchung erfasst“ (Vorsitzender 13).

Ein Vorsitzender gab sein Einverständnis zu der geplanten Studie nur,

„falls eine ausschließlich auf den Fall bezogene Analytik vorgenommen wird und weiteres Material irreversibel anonymisiert wird" (Vorsitzender 4).

Weitere Vorsitzende forderten eine 


\begin{tabular}{|c|c|c|c|c|}
\hline Kategorie & $\begin{array}{l}\text { Anzahl der } \\
\text { Zuordnungen } \\
\text { (insg. 21) }\end{array}$ & $\begin{array}{l}\text { Befür- } \\
\text { wortung } \\
\text { der } \\
\text { Studie }\end{array}$ & $\begin{array}{l}\text { Unent- } \\
\text { schieden }\end{array}$ & $\begin{array}{l}\text { Ableh- } \\
\text { nung }\end{array}$ \\
\hline Aufklärung der Eltern erforderlich & 4 & 4 & - & - \\
\hline $\begin{array}{l}\text { Einwilligung der Eltern } \\
\text { erforderlich }\end{array}$ & 4 & 4 & - & - \\
\hline $\begin{array}{l}\text { Wenn möglich, Einwilligung des } \\
\text { Kindes }\end{array}$ & 1 & 1 & - & - \\
\hline Keine Einwilligung erforderlich & 1 & 1 & - & - \\
\hline $\begin{array}{l}\text { Beschränkung der genetischen } \\
\text { Untersuchung auf die } \\
\text { Fragestellung erforderlich }\end{array}$ & 4 & 4 & - & - \\
\hline $\begin{array}{l}\text { Anonymisierung des Materials } \\
\text { erforderlich }\end{array}$ & 3 & 3 & - & - \\
\hline $\begin{array}{l}\text { Vernichtung der Proben } \\
\text { erforderlich }\end{array}$ & 2 & 2 & - & - \\
\hline $\begin{array}{l}\text { Hohe Anforderungen an } \\
\text { Patientenschutz und } \\
\text { wissenschaftliche Qualität }\end{array}$ & 1 & 1 & - & - \\
\hline
\end{tabular}

Tabelle 5.3: Vignette 1: Kategorisierung der Anmerkungen und ihre Häufigkeit, bezogen auf die gewählte Antwortmöglichkeit 
„irreversible Anonymisierung der Daten zum Schutz der Familie“ (Vorsitzender 22, auBerdem Vorsitzende 4 und 19).

Einigen war es wichtig, zu betonen, dass die Proben nach Abschluss der Analytik vernichtet werden sollten (Vorsitzende 6,8) und dass die genetische Untersuchung auf die vorgegebene Fragestellung beschränkt sein müsse. Sie forderten die

„Information, dass Untersuchung nur zu diesem Zweck erfolgt und Probe hernach verworfen wird" (Vorsitzender 8, außerdem Vorsitzende 1, 4 und 26).

Ein Vorsitzender äußerte die Ansicht, dass Forschung an „Restmaterial“ wie Blut nicht zustimmungspflichtig sei (Vorsitzender 15).

Ein weiterer verlangte

„hohe Anforderungen an Patientenschutz, hohe Anforderungen an Anonymisierung, hohe Anforderungen an wissenschaftliche Qualität" (Vorsitzender 19).

\subsubsection{Vignette 2: Psychokognitive Tests bei HKS und LRS}

\section{Quantitative Auswertung}

Abbildung 5.2 stellt dar, dass die Vorsitzenden mit der Durchführung der in Vignette 2 beschriebenen Studie im Prinzip einverstanden waren. Fünf Vorsitzende $(17,2 \%)$ stimmten der Studie mit Einschränkungen zu, und 24 Vorsitzende $(82,8 \%$ ) hatten keine Vorbehalte bezüglich ihrer Durchführung.

\section{Studie vertretbar? Positives Votum?}

Ja, ohne Einschränkungen

Ja, mit Einschränkungen

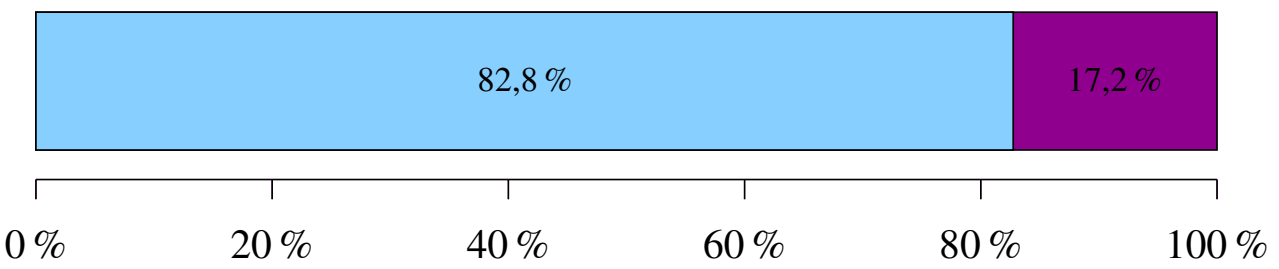

Anteil der Vorsitzenden $(\mathrm{N}=29)$

Abbildung 5.2: Vignette 2, Frage 1: „Halten Sie eine solche Studie für vertretbar und würden Sie ein positives Votum einer Ethikkommission befürworten?“ 


\section{Auswertung der Anmerkungen}

13 Vorsitzende $(44,8 \%)$ formulierten Anmerkungen und Begründungen zu ihrer Entscheidung (siehe Tabelle 5.4).

\begin{tabular}{lcccc}
\hline Kategorie & $\begin{array}{l}\text { Anzahl der } \\
\text { Zuordnungen } \\
\text { (insg. 23) }\end{array}$ & $\begin{array}{l}\text { Befür- } \\
\text { wortung } \\
\text { der } \\
\text { Studie }\end{array}$ & $\begin{array}{l}\text { Unent- } \\
\text { schieden }\end{array}$ & $\begin{array}{l}\text { Ableh- } \\
\text { nung }\end{array}$ \\
\hline $\begin{array}{l}\text { Abklärung der psychischen und } \\
\text { körperlichen Belastung des Kindes } \\
\text { durch die Studie erforderlich }\end{array}$ & $\mathbf{3}$ & 3 & - & - \\
$\begin{array}{l}\text { Aufklärung der Eltern erforderlich } \\
\begin{array}{l}\text { Einwilligung der Eltern } \\
\text { erforderlich }\end{array}\end{array}$ & $\mathbf{3}$ & 3 & - & - \\
$\begin{array}{l}\text { Aufklärung des Kindes erforderlich } \\
\begin{array}{l}\text { Einwilligung des Kindes } \\
\text { erforderlich }\end{array}\end{array}$ & $\mathbf{4}$ & 4 & - & - \\
$\begin{array}{l}\text { Zustimmung des Kindes } \\
\text { erforderlich }\end{array}$ & $\mathbf{2}$ & 2 & - & - \\
$\begin{array}{l}\text { Anonymisierung der Daten } \\
\text { erforderlich }\end{array}$ & $\mathbf{1}$ & 1 & - & - \\
$\begin{array}{l}\text { Anmerkungen zur Qualität der } \\
\text { Studie }\end{array}$ & $\mathbf{2}$ & 2 & - & - \\
\hline
\end{tabular}

Tabelle 5.4: Vignette 2: Kategorisierung der Anmerkungen und ihre Häufigkeit, bezogen auf die gewählte Antwortmöglichkeit

Viele Vorsitzende verwiesen auf die Bedeutung von Aufklärung (Vorsitzende 6, 11 und 29) und Einwilligung (Vorsitzende 11, 13, 14 und 15) der Eltern, Aufklärung (Vorsitzende 11, 24, 26 und 29) und Einwilligung (Vorsitzende 11 und 13) bzw. Zustimmung (Vorsitzender 24) der Kinder und die Notwendigkeit, die erhobenen Daten zu anonymisieren (Vorsitzende 6 und 22).

Einwände betrafen die Befürchtung, bei den Kindern könnte durch die Tests Stress oder Angst ausgelöst werden. Ein Vorsitzender forderte im Vorfeld der Studie eine

„genaue ärztliche Beurteilung, ob die Kinder körperlich und seelisch den Testanforderungen gewachsen sind" (Vorsitzender 9). 
Ein anderer Vorsitzender verlangte, die Testprozedur

„,darf keinen Stress oder Angst auslösen“

und das Kind habe einen Anspruch auf eine

„gute Erklärung, wann, wieso und wie“(Vorsitzender 17).

Vor Studienbeginn sei eine

„Abklärung der Belastung durch die EEG-Ableitung in der Diskussion mit den Studienleitern erforderlich" (Vorsitzender 26).

Mehrere Vorsitzende kommentierten die methodische Qualität der Studie (Vorsitzende 14, 16, 24 und 25). Eine dieser Anmerkungen beschäftigte sich mit der Rolle des Studienleiters und mit dem Vorgehen bei der Probandensuche. Ein Vorsitzender, der die Studie mit Einschränkungen befürwortete, formulierte folgende Anmerkung:

„Die Einschränkung betrifft die Qualität der Studie und der Untersucher, auch die Art der Kontrollgruppe. Wenn ein qualifizierter Neurowissenschaftler die Untersuchung durchführt und eigene Kinder und deren Freunde als Kontrolle verwendet: Ja. Wenn ein modischer Psychologe/Pädagoge sich Kontrollen aus der Schule fängt: Nein “ (Vorsitzender 25).

\subsubsection{Vignette 3: Orale Placebogabe mit Verzicht auf Standardtherapie bei allergischem Schnupfen}

\section{Quantitative Auswertung}

Abbildung 5.3 zeigt, dass die Vorsitzenden Vignette 3 überwiegend positiv bewerteten: Zehn Vorsitzende (34,5\%) würden die Studie ohne und weitere neun $(31,0 \%)$ mit Einschränkungen gutheißen. Zehn Vorsitzende $(34,5 \%)$ würden ihrer Durchführung auf gar keinen Fall zustimmen.

\section{Auswertung der Anmerkungen}

25 Vorsitzende $(86,2 \%)$ machten weitere Anmerkungen zu ihrer Entscheidung (siehe Tabelle 5.5).

Jeweils zwei Vorsitzende verwiesen in ihren Anmerkungen auf die Bedeutung der Aufklärung der Eltern (Vorsitzende 6 und 23) und des Kindes (Vorsitzende 6 und 26).

Viele Vorsitzende knüpften ihre Zustimmung an methodische Bedingungen zum Ablauf der Studie: Einige machten ihr positives Votum von der Definition von Abbruchkriterien (Vorsitzende 9, 10, 17 und 29) oder von einer Zwischenauswertung (Vorsitzender 7) abhängig. 
Studie vertretbar? Positives Votum?
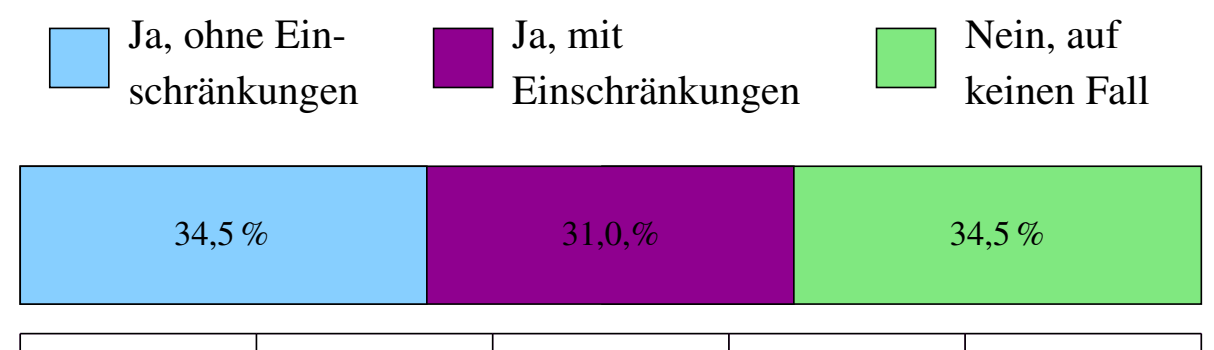

$\begin{array}{llllll}0 \% & 20 \% & 40 \% & 60 \% & 80 \% & 100 \%\end{array}$

Anteil der Vorsitzenden $(\mathrm{N}=29)$

Abbildung 5.3: Vignette 3, Frage 1: „Halten Sie eine solche Studie für vertretbar und würden Sie ein positives Votum einer Ethikkommission befürworten?“

Zwei Vorsitzende würden die Studie nur dann positiv bewerten, wenn die Kinder und Jugendlichen der Placebogruppe im Notfall auf die bisher üblicherweise verwendeten Medikamente zurückgreifen könnten (Vorsitzende 3 und 12).

Weitere Vorsitzende stellten die Einbeziehung Minderjähriger bei dieser Studie in Frage. Sie stimmten dieser Studie unter der Bedingung zu, dass die Therapie bereits erfolgreich mit Erwachsenen getestet sei und diese Ergebnisse nicht auf Kinder und Jugendliche übertragbar seien (Vorsitzende 7, 8, 19 und 22).

Ein Vorsitzender betonte die Bedeutung einer sorgfältigen Probandenauswahl, gerade im Falle einer placebokontrollierten Phase-2-Studie:

„Bei den Einschlusskriterien müssen bestimmte Schweregrade ausgeschlossen bleiben, ansonsten Testung gegen eine zugelassene Referenzsubstanz" (Vorsitzender 29).

Ein Vorsitzender äußerte kritisch, dass ein Placeboeinsatz nur dann zu rechtfertigen sei, „wenn die unzuverlässige Medikation nicht als Kontrollgröße dienen kann“ (Vorsitzender $16)$.

Eindeutige Angaben zur zu prüfenden Substanz müssten vorliegen (Vorsitzender 29).

Zwei Vorsitzende hatten rechtliche Bedenken bezüglich der Studie.

„Die Studie ist nach $A M G^{36}$ nicht zulässig, da die Wirksamkeit des neuen Histaminikums auch an Patienten >18 Jahre geprüft werden kann " (Vorsitzender 11).

Sie sei

${ }^{36}$ Zum Zeitpunkt der Datenerhebung galt das AMG von 1994. 


\begin{tabular}{|c|c|c|c|c|}
\hline Kategorie & $\begin{array}{l}\text { Anzahl der } \\
\text { Zuordnungen } \\
\text { (insg. 28) }\end{array}$ & $\begin{array}{l}\text { Befür- } \\
\text { wortung } \\
\text { der } \\
\text { Studie }\end{array}$ & $\begin{array}{l}\text { Unent- } \\
\text { schieden }\end{array}$ & $\begin{array}{l}\text { Ableh- } \\
\text { nung }\end{array}$ \\
\hline $\begin{array}{l}\text { Prüfung nur gegen } \\
\text { Standardtherapie }\end{array}$ & 7 & - & - & 7 \\
\hline $\begin{array}{l}\text { Keine placebokontrollierten } \\
\text { Studien an Kindern }\end{array}$ & 1 & - & - & 1 \\
\hline Studie rechtlich nicht zulässig & 2 & - & - & 2 \\
\hline Aufklärung der Eltern erforderlich & 2 & 2 & - & - \\
\hline Aufklärung des Kindes erforderlich & 2 & 2 & - & - \\
\hline $\begin{array}{l}\text { Studien an Erwachsenen müssen } \\
\text { vorliegen und Ergebnisse dürfen } \\
\text { nicht auf Kinder übertragbar sein }\end{array}$ & 4 & 4 & - & - \\
\hline $\begin{array}{l}\text { Definition von Abbruchkriterien } \\
\text { erforderlich }\end{array}$ & 4 & 4 & - & - \\
\hline Zwischenauswertung erforderlich & 1 & 1 & - & - \\
\hline $\begin{array}{l}\text { Sorgfältige Auswahl der } \\
\text { Probanden erforderlich }\end{array}$ & 1 & 1 & - & - \\
\hline $\begin{array}{l}\text { Zurückgreifen auf üblicherweise } \\
\text { verwendete Therapie soll möglich } \\
\text { sein }\end{array}$ & 2 & 2 & - & - \\
\hline $\begin{array}{l}\text { Üblicherweise verwendete } \\
\text { Therapie sollte, wenn möglich, als } \\
\text { Kontrollgröße dienen }\end{array}$ & 1 & 1 & - & - \\
\hline $\begin{array}{l}\text { Eindeutige Angaben zur Substanz } \\
\text { müssen vorliegen }\end{array}$ & 1 & 1 & - & - \\
\hline
\end{tabular}

Tabelle 5.5: Vignette 3: Kategorisierung der Anmerkungen und ihre Häufigkeit, bezogen auf die gewählte Antwortmöglichkeit 
„laut AMG $\S 40^{37}$ nicht möglich“ (Vorsitzender 27).

Sieben der zehn Vorsitzenden, die die Studiendurchführung abgelehnt hatten, begründen diese Entscheidung damit, dass es nicht zumutbar sei, die neue Substanz gegen Placebo zu testen und dabei auf die Standardtherapie zu verzichten.

„Prüfung gegen Standardtherapie möglich; sechs Monate ohne Therapie nicht zumutbar" (Vorsitzender 13, außerdem Vorsitzende 5, 14, 15, 20, 24 und 25).

Ein weiterer Vorsitzender forderte, grundsätzlich

„,keine placebokontrollierten Studien (randomisiert) bei Kindern“ (Vorsitzender 21)

durchzuführen.

\subsubsection{Vignette 4: Herzmuskelbiopsie bei ohnehin notwendiger Herzoperation}

\section{Quantitative Auswertung}

Die Abbildung 5.4 in dargestellten Ergebnisse zeigen, dass bei der Bewertung von Vignette 4 die Ansichten der Vorsitzenden auseinander gingen: Elf Vorsitzende $(37,9 \%)$ befürworteten die Durchführung der Studie ohne Einschränkungen. Vier Vorsitzende $(13,8 \%)$ wären mit Einschränkungen damit einverstanden. Die Antwortmöglichkeit „Ich weiß nicht/keine Meinung “wählten sechs Vorsitzende (20,7\%), während sich acht Vorsitzende $(27,6 \%)$ gegen eine Durchführung der in der Vignette vorgestellten Studie aussprachen.

\section{Auswertung der Anmerkungen zu Frage 1}

25 Vorsitzende $(86,2 \%)$ nutzten die Möglichkeit, ihre Entscheidung zu kommentieren (siehe Tabelle 5.6).

Als Anmerkungen wurden die Notwendigkeit und Bedeutung von ausführlicher Aufklärung (Vorsitzende 2, 6, 23 und 24) und Einwilligung der Eltern (Vorsitzende 14, 17, 22, 23 und 24) im Sinne des informed consent genannt.

Zwei Vorsitzende gaben an, dass ihrer Ansicht nach die Biopsie nur dann durchgeführt werden könne, wenn es nicht möglich sei, die Fragestellung durch Forschung an älteren Kindern oder Erwachsenen zu bearbeiten (Vorsitzende 16 und 26). Zwei weitere Vorsitzende schlugen vor, im Rahmen der Operation anfallendes Material zu nutzen und auf die Gewebebiopsie zu verzichten (Vorsitzende 7 und 11).

Vorsitzende, die angaben, die Studie nicht beurteilen zu können, wünschten sich meist mehr Informationen zum wissenschaftlichen Zweck und zur Herzmuskelbiopsie. Es fiel

${ }^{37}$ Zum Zeitpunkt der Datenerhebung galt das AMG von 1994. 
Studie vertretbar? Positives Votum?

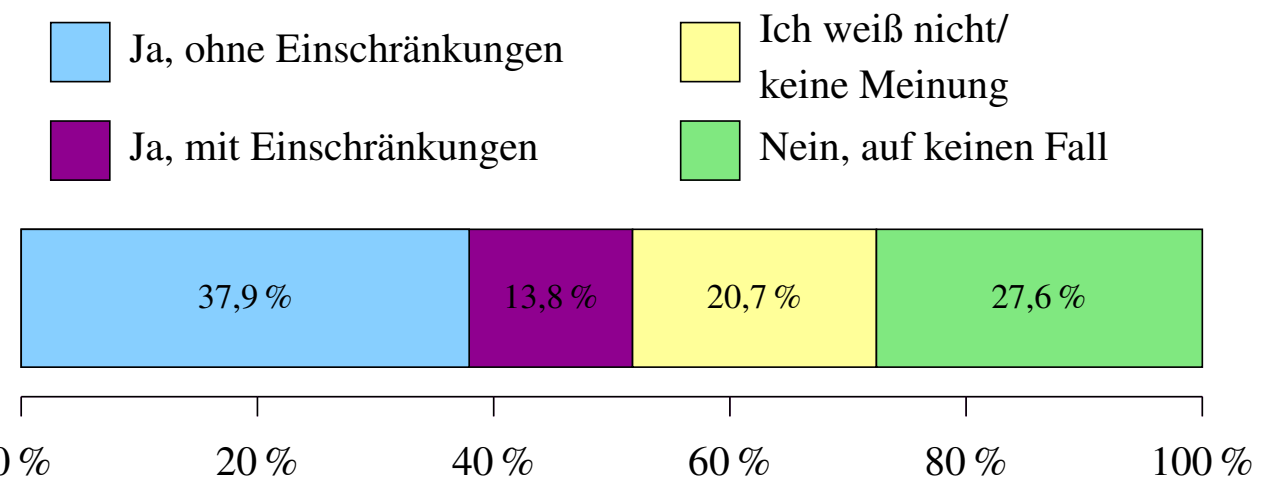

Anteil der Vorsitzenden ( $\mathrm{N}=29)$

Abbildung 5.4: Vignette 4, Frage 1: „Halten Sie eine solche Studie für vertretbar und würden Sie ein positives Votum einer Ethikkommission befürworten?“

ihnen offensichtlich schwer, das Risiko dieser Intervention einzuschätzen (Vorsitzende 10, 13, 18, 19, 21 und 29). Ein Vorsitzender betonte dabei, dass eine solche Entscheidung nie von ihm allein getroffen werden könne, sondern dass Information durch Experten nötig sei, um das Risiko eines solchen Eingriffs einschätzen zu können:

„... kann alleine diese Frage nicht beantworten. Das geht nur nach Diskussion in der Kommission mit einem Kinderkardiologen und/oder Herzchirurgen. Der Vorsitzende einer Ethikkommission hat kein alleiniges Entscheidungsrecht" (Vorsitzender 21).

Sieben Vorsitzende machten Bemerkungen zum wissenschaftlichen Sinn der Biopsie (1, 4, 8, 12, 15, 24 und 25), und vier Vorsitzende kommentierten den Einsatz des Betarezeptorenblockers aus medizinischer Perspektive (Vorsitzende 6, 7, 22 und 25).

Oft wurde geäußert, dass bei dem schwer einschätzbaren Biopsierisiko die Studie besonders genau geplant werden müsse und eine sorgfältige Nutzen-Risiko-Analyse für ihre Durchführung Voraussetzung sei. So wurde z. B. eine

„klare Angabe und Begründung der Anzahl von Herzmuskelbiopsien und der RisikenNutzen-Kalkulation in der Aufklärung " (Vorsitzender 1, außerdem Vorsitzende 13 und 16)

gefordert.

Drei Vorsitzende lehnten die Biopsie aufgrund fehlenden Nutzens für das Kind mit folgenden Begründungen $\mathrm{ab}$ :

„Anders könnte die Entscheidung ausfallen, wenn die Myokardbiopsie hinsichtlich der weiteren Therapie von Bedeutung wäre" (Vorsitzender 3). 


\begin{tabular}{|c|c|c|c|c|}
\hline Kategorie & $\begin{array}{l}\text { Anzahl der } \\
\text { Zuordnungen } \\
\text { (insg. 33) }\end{array}$ & $\begin{array}{l}\text { Befür- } \\
\text { wortung } \\
\text { der } \\
\text { Studie }\end{array}$ & $\begin{array}{l}\text { Unent- } \\
\text { schieden }\end{array}$ & $\begin{array}{l}\text { Ableh- } \\
\text { nung }\end{array}$ \\
\hline $\begin{array}{l}\text { Potentieller Nutzen der Biopsie ist } \\
\text { nicht gegeben }\end{array}$ & 3 & - & - & 3 \\
\hline $\begin{array}{l}\text { Sorgfältige } \\
\text { Nutzen-Risiko-Abwägung } \\
\text { erforderlich }\end{array}$ & 3 & 2 & 1 & - \\
\hline Aufklärung der Eltern erforderlich & 4 & 4 & - & - \\
\hline $\begin{array}{l}\text { Einwilligung der Eltern } \\
\text { erforderlich }\end{array}$ & 5 & 4 & - & 1 \\
\hline $\begin{array}{l}\text { Studien an Erwachsenen müssen } \\
\text { vorliegen und Ergebnisse dürfen } \\
\text { nicht auf Kinder übertragbar sein }\end{array}$ & 2 & 2 & - & - \\
\hline $\begin{array}{l}\text { Für Entscheidung mehr } \\
\text { Informationen zum Risiko der } \\
\text { Biopsie erforderlich }\end{array}$ & 6 & - & 5 & 1 \\
\hline $\begin{array}{l}\text { Biopsie nur aus anfallendem } \\
\text { OP-Material }\end{array}$ & 2 & - & - & 2 \\
\hline $\begin{array}{l}\text { Anmerkung zum } \\
\text { wissenschaftlichen Sinn der } \\
\text { Biopsie }\end{array}$ & 7 & 4 & - & 3 \\
\hline Arzneimittelstudie akzeptabel & 1 & 1 & - & - \\
\hline $\begin{array}{l}\text { Einsatz des Betarezeptorenblockers } \\
\text { muss begründet werden }\end{array}$ & 2 & 2 & - & - \\
\hline $\begin{array}{l}\text { Betarezeptorenblocker } \\
\text { wissenschaftlich nicht sinnvoll }\end{array}$ & 1 & - & - & 1 \\
\hline
\end{tabular}

Tabelle 5.6: Vignette 4: Kategorisierung der Anmerkungen und ihre Häufigkeit, bezogen auf die gewählte Antwortmöglichkeit 
„Eine Herzmuskelbiopsie würde ich nicht befürworten, da ein möglicher Nutzen nicht gegeben ist" (Vorsitzender 14, außerdem Vorsitzender 15).

\subsubsection{Vignette 5: Intramuskuläre Placeboinjektionen bei Kindern mit angeborenem Herzfehler}

\section{Quantitative Auswertung}

Abbildung 5.5 zeigt, dass sieben Vorsitzende $(24,1 \%)$ mit der Umsetzung der in Vignette 5 vorgestellten Studienplanung ohne und sieben weitere Vorsitzende $(24,1 \%)$ mit Einschränkungen einverstanden wären. Drei Vorsitzende $(10,3 \%)$ waren sich unsicher und zwölf Vorsitzende $(41,4 \%)$ würden ihre Durchführung auf gar keinen Fall befürworten.

\section{Studie vertretbar? Positives Votum?}
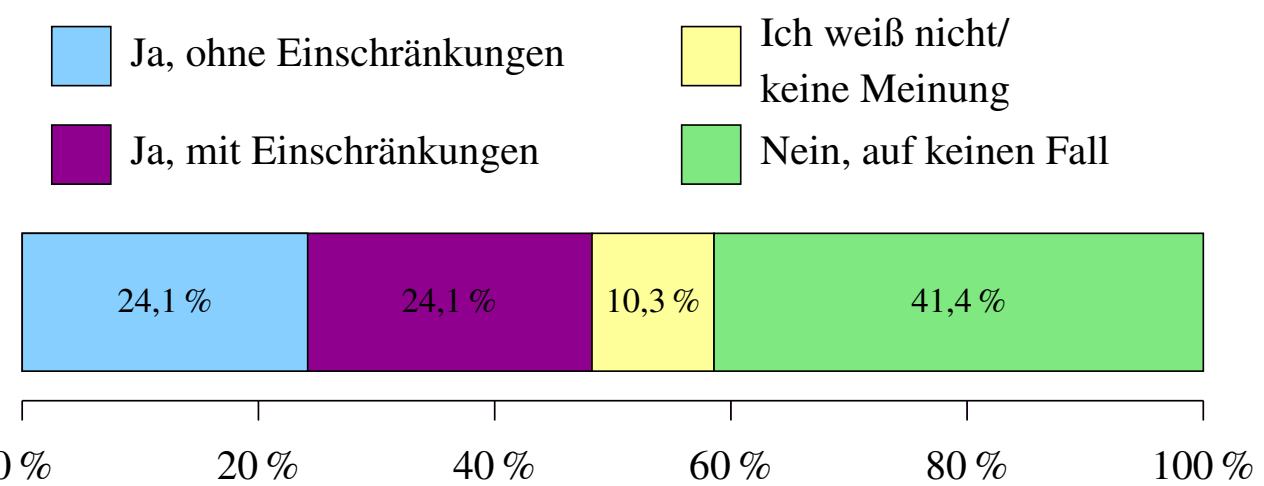

Anteil der Vorsitzenden $(\mathrm{N}=29)$

Abbildung 5.5: Vignette 5, Frage 1: „Halten Sie eine solche Studie für vertretbar und würden Sie ein positives Votum einer Ethikkommission befürworten?“

\section{Auswertung der Anmerkungen}

22 Vorsitzende (75,9\%) nutzten die Möglichkeit, Anmerkungen zu ihrer Entscheidung zu formulieren (siehe Tabelle 5.7).

Sie betonten dabei die Notwendigkeit von Aufklärung (Vorsitzender 15) und Einwilligung (Vorsitzende 14 und 15) der Eltern betont.

Einige Vorsitzende machten ihre Zustimmung von der erfolgreichen Prüfung des Medikaments an Erwachsenen abhängig.

„Nur wenn bei Untersuchungen an Erwachsenen der Nachweis der prophylaktischen Wirkung geführt wurde“ (Vorsitzender 22), 


\begin{tabular}{|c|c|c|c|c|}
\hline Kategorie & $\begin{array}{l}\text { Anzahl der } \\
\text { Zuordnungen } \\
\text { (insg. 24) }\end{array}$ & $\begin{array}{l}\text { Befür- } \\
\text { wortung } \\
\text { der } \\
\text { Studie }\end{array}$ & $\begin{array}{l}\text { Unent- } \\
\text { schieden }\end{array}$ & $\begin{array}{l}\text { Ableh- } \\
\text { nung }\end{array}$ \\
\hline Placeboarm nicht vertretbar & 5 & - & - & 5 \\
\hline Studie rechtlich nicht zulässig & 1 & - & - & 1 \\
\hline $\begin{array}{l}\text { Für Entscheidung mehr } \\
\text { Informationen zur } \\
\text { Nutzen-Risiko-Abwägung } \\
\text { erforderlich }\end{array}$ & 1 & - & 1 & - \\
\hline Aufklärung der Eltern erforderlich & 1 & 1 & - & - \\
\hline $\begin{array}{l}\text { Einwilligung der Eltern } \\
\text { erforderlich }\end{array}$ & 2 & 1 & - & 1 \\
\hline $\begin{array}{l}\text { Studien an Erwachsenen müssen } \\
\text { vorliegen und Ergebnisse dürfen } \\
\text { nicht auf Kinder übertragbar sein }\end{array}$ & 2 & 1 & 1 & - \\
\hline $\begin{array}{l}\text { Definition von Abbruchkriterien } \\
\text { erforderlich }\end{array}$ & 1 & 1 & - & - \\
\hline $\begin{array}{l}\text { Wissenschaftliche Begründung } \\
\text { muss überzeugend sein }\end{array}$ & 1 & 1 & - & - \\
\hline Placeboarm unnötig & 3 & - & - & 3 \\
\hline Placeboarm notwendig & 1 & 1 & - & - \\
\hline Studie wissenschaftlich unklar & 6 & - & 1 & 5 \\
\hline
\end{tabular}

Tabelle 5.7: Vignette 5: Kategorisierung der Anmerkungen und ihre Häufigkeit, bezogen auf die gewählte Antwortmöglichkeit 
dürfe die Studie so durchgeführt werden (außerdem Vorsitzender 19).

Ein Vorsitzender betonte die Notwendigkeit, die Versuchsperson bei der Studiendurchführung zu schützen, und würde die Studiendurchführung dann befürworten,

„wenn die Erkrankung frühzeitig erkannt und behandelt werden kann. Abbruchkriterien für die Studie müssen definiert werden " (Vorsitzender 16).

Ein anderer würde sie gutheißen,

„wenn die Wissenschaftlichkeit der Studie gewährleistet ist“ (Vorsitzender 6).

Die Vorsitzenden äußerten sich unterschiedlich zu den Placeboinjektionen. Teils wurden diese für notwendig gehalten, denn

„aus Mangel an Alternativen wäre Placeboarm nicht zu umgehen“ (Vorsitzender 15),

teils aber auch für überflüssig:

„Die Kochsalzinjektionen erscheinen mir unnötig “ (Vorsitzender 3, außerdem Vorsitzende 10 und 13).

Mehrere Vorsitzende halten Placeboinjektionen bei Kindern grundsätzlich für nicht akzeptabel:

„Placeboarm (i.m. Injektionen) nicht vertretbar“ (Vorsitzender 7).

„Ferner ist es ethisch nicht vertretbar, dass die Kontrollgruppe physiologische Kochsalzlösung injiziert bekommt" (Vorsitzender 14).

„Keine placebokontrollierten Studien mit Kleinkindern“(Vorsitzender 21).

„Placebo-Injektionen würde ich bei Kindern dieser Altersgruppe auch in weniger unsinnigen Studien nicht akzeptieren “ (Vorsitzender 25, außerdem Vorsitzender 18).

„Laut AMG $\S 40^{38}$ nicht möglich“(Vorsitzender 27).

Einige Vorsitzende lehnten die Studie auch deshalb ab, weil sie ihnen wissenschaftlich unklar blieb.

„Die Immunglobulin-Injektionen machen keinen Sinn bei Herzmuskelentzündungen, die durch RS-Viren hervorgerufen werden " (Vorsitzender 14, außerdem Vorsitzende 7, 9, 24, 25 und 29).

Eine Nutzen-Risiko-Abwägung sei anhand der Angaben schwierig (Vorsitzender 11).

${ }^{38}$ Zum Zeitpunkt der Datenerhebung galt das AMG von 1994. 


\subsubsection{Vignette 6: Zusätzliche Knochenmarkpunktionen bei ALL}

\section{Quantitative Auswertung}

In Abbildung 5.6 ist dargestellt, wie die Vorsitzenden über das in Vignette 6 vorgestellte Forschungsprojekt entscheiden würden. Sechs Vorsitzende $(20,7 \%)$ würden eine Durchführung dieser Studie ohne Einschränkung befürworten und weitere sechs $(20,7 \%)$ ein positives Votum an bestimmte Einschränkungen knüpfen. Der überwiegende Teil der Vorsitzenden beurteilte die Versuchsplanung sehr skeptisch. 17 Vorsitzende $(58,6 \%)$ würden sich in keinem Fall für eine Durchführung der Studie in dieser Form aussprechen.

Studie vertretbar? Positives Votum?
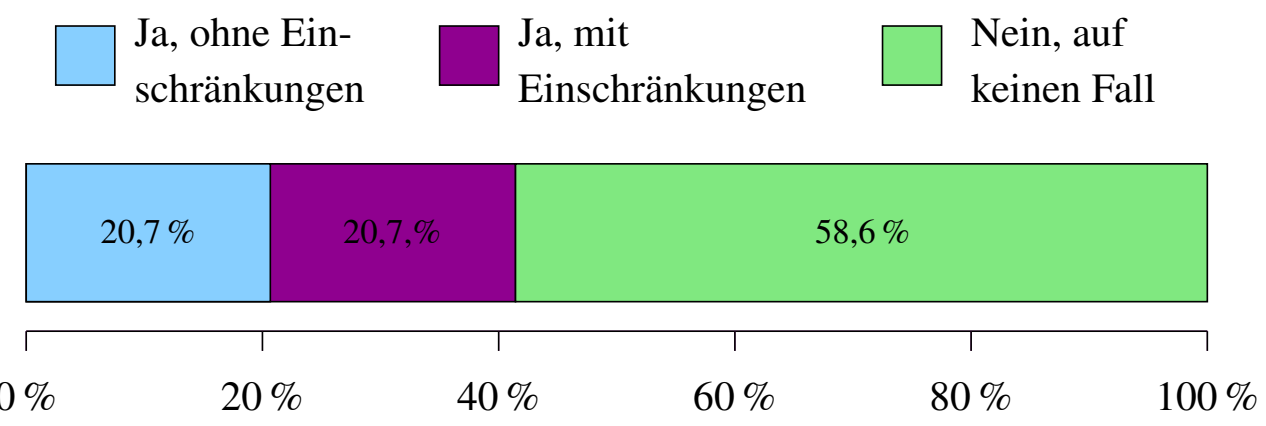

Anteil der Vorsitzenden ( $\mathrm{N}=29)$

Abbildung 5.6: Vignette 6, Frage 1: „Halten Sie eine solche Studie für vertretbar und würden Sie ein positives Votum einer Ethikkommission befürworten?“

\section{Auswertung der Anmerkungen}

21 Vorsitzende $(72,4 \%)$ nutzten die Gelegenheit, ihre Entscheidung zu begründen oder Anmerkungen zu der Vignette zu machen (siehe Tabelle 5.8).

In den Anmerkungen betonten die Vorsitzenden die Bedeutung von Aufklärung (Vorsitzende 2, 11 und 23) und Einwilligung (Vorsitzende 2, 11 und 14) der Eltern und Aufklärung (Vorsitzende 11 und 26) und Zustimmung (Vorsitzender 11) des Kindes.

Ein Vorsitzender bat dabei um eine kindgerechte Darstellung der Nutzen-Risiko-Berechnung und forderte,

„auch den Kindern genaue Darlegung der möglichen Vorteile und Nachteile“ (Vorsitzender 26) zu geben.

Drei Vorsitzende würden die Studie bei überzeugender wissenschaftlicher Begründung gutheißen: 


\begin{tabular}{|c|c|c|c|c|}
\hline Kategorie & $\begin{array}{l}\text { Anzahl der } \\
\text { Zuordnungen } \\
\text { (insg. 32) }\end{array}$ & $\begin{array}{l}\text { Befür- } \\
\text { wortung } \\
\text { der } \\
\text { Studie }\end{array}$ & $\begin{array}{l}\text { Unent- } \\
\text { schieden }\end{array}$ & $\begin{array}{l}\text { Ableh- } \\
\text { nung }\end{array}$ \\
\hline $\begin{array}{l}\text { Zusätzliche Punktionen nicht } \\
\text { vertretbar, Belastung zu groß }\end{array}$ & 9 & - & - & 9 \\
\hline $\begin{array}{l}\text { Studie vertretbar bei guter } \\
\text { wissenschaftlicher Begründung }\end{array}$ & 3 & 3 & - & - \\
\hline $\begin{array}{l}\text { Sorgfältige } \\
\text { Nutzen-Risiko-Abwägung } \\
\text { erforderlich }\end{array}$ & 1 & 1 & - & - \\
\hline Kein potentieller direkter Nutzen & 2 & - & - & 2 \\
\hline Aufklärung der Eltern erforderlich & 3 & 2 & - & 1 \\
\hline $\begin{array}{l}\text { Einwilligung der Eltern } \\
\text { erforderlich }\end{array}$ & 3 & 1 & - & 2 \\
\hline Aufklärung des Kindes erforderlich & 2 & - & - & 2 \\
\hline $\begin{array}{l}\text { Zustimmung des Kindes } \\
\text { erforderlich }\end{array}$ & 1 & - & - & 1 \\
\hline Studie wissenschaftlich unklar & 5 & 1 & - & 4 \\
\hline Bitte nach Alternativen suchen & 3 & 1 & - & 2 \\
\hline
\end{tabular}

Tabelle 5.8: Vignette 6: Kategorisierung der Anmerkungen und ihre Häufigkeit, bezogen auf die gewählte Antwortmöglichkeit 
„Es müsste detailliert begründet werden, welcher wissenschaftliche Zugewinn durch die überzähligen Punktionen erwartet wird und zu welchen Konsequenzen er führen könnte“ (Vorsitzender 27).

„Nachweis der zusätzlichen Punktion bzw. des zusätzlichen Erkenntnisgewinns fehlt. Erst wenn die absolute Notwendigkeit der sechs zusätzlichen Punktionen vorliegt, evtl. Zustimmung " (Vorsitzender 17, außerdem Vorsitzender 15).

Ein Vorsitzender forderte eine sehr sorgfältige Nutzen-Risiko-Kalkulation:

„Höchste Messlatte, da invasiv und schwer belastend; nur dann, wenn belegt, dass Erkenntnisgewinn nicht anders zu gewinnen ist und Risiko-Nutzen-Relation stimmt " (Vorsitzender 26).

Drei Vorsitzende würden den Antragstellern empfehlen, nach Alternativen zu suchen und die vorgeschlagene Anzahl invasiver Eingriffe zu reduzieren (Vorsitzende 13, 14 und $18)$.

Zwei Vorsitzende lehnten die Studie dagegen mit der Begründung ab, dass es sich um eine Untersuchung ohne potentiellen direkten Nutzen handele, die sie nicht befürworten könnten (Vorsitzende 6 und 25).

Neun Vorsitzende empfanden die Belastung für das einzelne Kind als zu groß (Vorsitzende 3, 7, 11, 14, 18, 20, 22, 25 und 26). Die Knochenmarkpunktionen seien eine

„unzumutbare Belästigung nur zum Erkenntnisgewinn“(Vorsitzender 7).

„Sechs KM-Biopsien zu wissenschaftlichen Zwecken sind den Kindern nicht zuzumuten“ (Vorsitzender 20).

Ein Vorsitzender kritisierte die Punktionen sogar als Kindesmisshandlung:

„Therapieoptimierungsstudien sind nötig und gut. Knochenmarkpunktionen ohne klinische Indikation sind Kindesmisshandlung und daher verboten. Wöchentliche Punktionen sind auch unter dem Deckmantel einer ,Verlaufskontrolle" nicht akzeptabel" (Vorsitzender 25).

Einigen weiteren Vorsitzenden erschien die Studie wissenschaftlich nicht plausibel (Vorsitzende 8, 10, 12, 24 und 29).

\subsubsection{Zusammenfassung der Auswertungen zur Zulässigkeit}

Abbildung 5.7 zeigt eine Übersicht der quantitativen Auswertung zur Zulässigkeit des jeweiligen in den Vignetten dargestellten Studientyps. 
Übersicht: Studie vertretbar? Positives Votum?

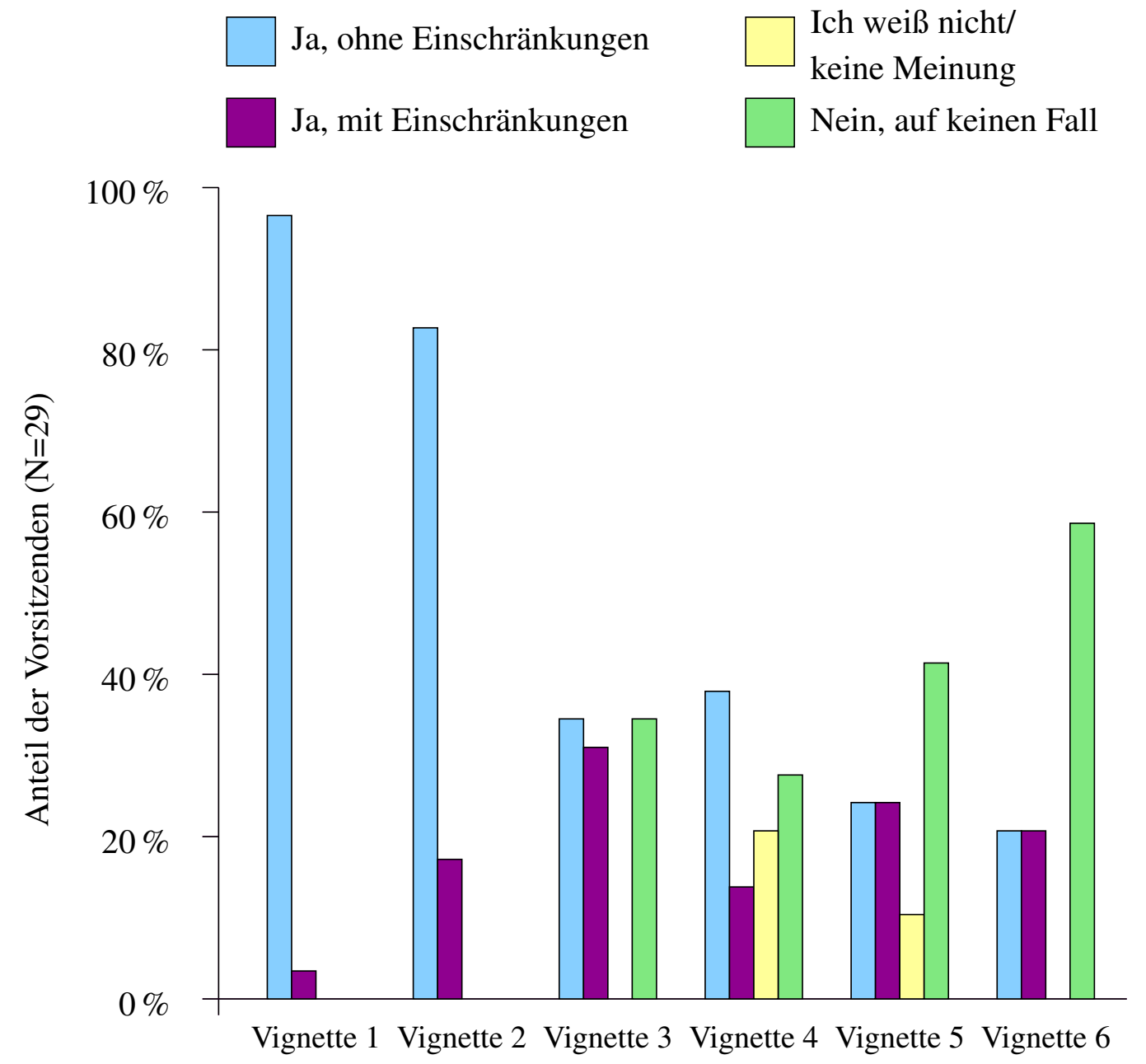

Abbildung 5.7: Übersicht Frage 1: „Halten Sie eine solche Studie für vertretbar und würden Sie ein positives Votum einer Ethikkommission befürworten?“ 
Insgesamt war das Antwortspektrum zu Vignette 1: Venöse Blutentnahme ohne Notwendigkeit zusätzlicher Punktion bei Rett-Syndrom (keine AMG-Studie, enge Gruppennützigkeit, minimales Risiko, minimale Belastung, zwei- bis fünfjährige, mental retardierte Kinder) homogen. Bezüglich der Zulässigkeit dieses Typs von Forschung bestanden keine Bedenken. Diese Vignette fand die fast uneingeschränkte Akzeptanz unter den Vorsitzenden der deutschen Ethikkommissionen.

In ihren Kommentaren legten die Vorsitzenden Wert darauf, dass Aufklärungs- und Einwilligungsprozeduren mit den Eltern bzw. gesetzlichen Vertretern korrekt vonstatten gingen, und betonten die besondere Notwendigkeit, im Hinblick auf die Untersuchung genetischen Materials den Datenschutz zu wahren und die Forschungen auf die Fragestellung zu begrenzen. Ethische Einwände gegen Forschung an geistig behinderten Kindern, wie Dörner und Spielmann sie bei der Analyse des Würzburger „Eisingen-Falls“ vorbringen (Dörner und Spielmann 2001), wurden nicht thematisiert.

Die Einstellung gegenüber Vignette 2: Psychokognitive Tests bei HKS und LRS (keine AMG-Studie, erweiterte und enge Gruppennützigkeit, minimales Risiko, minimale Belastung, sechs- bis elfjährige Kinder) war ähnlich positiv. Kein Vorsitzender hatte grundsätzlich Probleme mit diesem Typ von Forschung, deren Kategorisierung unter minimale Belastung an bestimmte Bedingungen (spielerische, kindgerechte Atmosphäre ohne Verletzung von Schamgefühlen) geknüpft ist. Genau auf diese Bedingungen verwiesen Vorsitzende in ihren Kommentaren und forderten eine genaue Abklärung der Belastung durch die Studienteilnahme.

Die Ergebnisse der Befragung zu Vignette 3: Orale Placebogabe mit Verzicht auf Standardtherapie bei allergischem Schnupfen (AMG-Studie, enge Gruppennützigkeit, mehr als minimales Risiko, mehr als minimale Belastung, zwölf- bis 18-jährige Kinder/Jugendliche, Randomisierung und Placebogabe mit Verzicht auf unzuverlässige Standardtherapie) zeigen, dass ca. ein Drittel (10/34,5\%) der Vorsitzenden diesen Studientyp nicht akzeptierten. Sie zweifelten die Zulässigkeit der Placebogabe an. Etwa zwei Drittel $(19 / 65,5 \%)$ der Vorsitzenden würden die Durchführung der Studie befürworten.

Vignette 4: Herzmuskelbiopsie bei ohnehin notwendiger Herzoperation (anteilig AMGStudie, enge Gruppennützigkeit, mehr als minimales Risiko, minimale Belastung, zwei Wochen bis drei Monate alte Babys, Randomisierung in Studien- und Kontrollgruppe) löste die größte Unsicherheit unter den Vorsitzenden aus. Sechs Vorsitzende (20,7\%) gaben an, mit den vorgegebenen Informationen nicht über die Zulässigkeit der Studie entscheiden zu können. Die Ursache dafür war aus den Kommentaren herauszulesen: Das Risiko der Herzmuskelbiopsie war für sie schlecht abschätzbar. Etwa die Hälfte der Vorsitzenden würde den vorgestellten Studientyp befürworten. Mehrere Vorsitzende forderten dabei eine sorgfältige Nutzen-Risiko-Abwägung, von der sie anscheinend ihre positive Entscheidung abhängig machten. Die übrigen Vorsitzenden waren gegen eine Studiendurchführung, wobei der überwiegende Teil dieser Antworten mit dem mehr als minimalen Risiko der Herzmuskelbiopsie begründet wurde. 
Die Ergebnisse zu Vignette 5: Intramuskuläre Placeboinjektionen bei angeborenem Herzfehler(AMG-Studie, enge Gruppennützigkeit, mehr als minimales Risiko, mehr als minimale Belastung, zwei- bis fünfjährige Kinder, Randomisierung und invasive Placebogabe) zeigten ein breites Spektrum: Etwa die Hälfte der Vorsitzenden würde die Studie befürworten (14 Vorsitzende/48,2 \%), ein etwas geringerer Teil (12 Vorsitzende/41,4\%) würde sie ablehnen. Die Ablehnung wurde zu einem Teil der Fälle mit einer nach Auffassung einiger Vorsitzender fraglichen wissenschaftlichen Plausibilität der Studie und zu einem anderen Teil mit dem zu hohen Risiko bzw. der zu hohen Belastung der Placeboinjektionen begründet. Drei Vorsitzende waren sich unsicher (10,3\%).

Am deutlichsten abgelehnt wurde der in Vignette 6: Zusätzliche Knochenmarkpunktionen bei ALL (AMG-Studie, enge Gruppennützigkeit, mehr als minimales Risiko, mehr als minimale Belastung, sechs- bis zehnjährige, leukämiekranke Kinder) vorgestellte Studientyp. Die zusätzlichen Knochenmarkpunktionen schienen den meisten Vorsitzenden ein zu großes Risiko und eine zu große Belastung für die leukämiekranken Kinder zu sein. Trotzdem würden zwölf Vorsitzende $(41,4 \%)$ die hier vorgestellte Studie mit oder ohne Einschränkungen befürworten.

Insgesamt wird deutlich, dass alle Vorsitzenden gruppennützige Forschung an Kindern und Jugendlichen akzeptierten. Gruppennützige Forschung mit minimalem Risiko und minimaler Belastung wird einheitlich für zulässig und vetretbar gehalten. Die Ergebnisse zu dem Studientyp mit mehr als minimalem Risiko (Vignette 4) zeigen ein heterogenes Meinungsspektrum. Geht neben dem mehr als minimalen Risiko auch eine mehr als minimale Belastung mit dem Studientyp einher, so empfindet ihn ein zunehmender Anteil der Vorsitzenden als nicht mehr vertretbar.

Als Gründe für eine Ablehnung der in den Vignetten vorgestellten Forschungsprojekte wurden verschiedene Argumente genannt. Dazu gehörten z. B.

- eine zu große psychische und physische Belastung durch bestimmte Interventionen,

- ein im Verhältnis zum potentiellen Nutzen der Studie zu großes Risiko und die Tatsache, dass

- Untersuchungen an Minderjährigen ohne potentiell eigennützigen Anteil ebenso wie

- placebokontrollierte Studien an Minderjährigen nicht für zulässig erachtet wurden.

Eine nicht unbeträchtliche Anzahl von Vorsitzenden würde allerdings auch Studien mit mehr als minimalem Risiko und mehr als minimaler Belastung bei guter wissenschaftlicher Begründung gutheißen. 


\subsubsection{Bedeutung der Beteiligung des Minderjährigen an Entscheidungen über seine Versuchsteilnahme}

Im Folgenden werden die Ergebnisse zu Frage 2 „Halten Sie eine individuelle Aufklärung und Zustimmung des Kindes für erforderlich?“, zu Frage 3 „Wäre für Sie ein eindeutig abwehrendes Verhalten des Kindes ein Ausschluss- bzw. Abbruchkriterium?" und zu Frage 4 ,Halten Sie eine Aufwandsentschädigung für das Kind für notwendig?“ dargestellt. Dabei werden, in der Reihenfolge der Vignetten geordnet, die Resultate der quantitativen Auswertung gezeigt.

Anschließend werden die Ergebnisse der Fragen zur Beteiligung des Minderjährigen an Entscheidungen über seine Versuchsteilnahme mit Bezug auf die einzelnen in den Vignetten dargestellten Studientypen verglichen.

\subsubsection{Vignette 1: Venöse Blutentnahme ohne Notwendigkeit zusätzlicher Punktion bei Rett-Syndrom}

\section{Aufklärung/Zustimmung (Frage 2)}

Abbildung 5.8 zeigt die Antworten der Vorsitzenden, die die o. g. Studie befürworteten $(100 \%, \mathrm{~N}=29)^{39}$, auf die Frage, ob sie eine individuelle Aufklärung und Zustimmung des Kindes für erforderlich hielten.

Neun Vorsitzende $(31,0 \%)$ hielten auch bei den mental retardierten Kindern im Alter von zwei bis fünf Jahren eine individuelle Aufklärung und Zustimmung für erforderlich. 16 Vorsitzende $(55,2 \%)$ sahen dafür in diesem Fall keine Notwendigkeit, und vier Vorsitzende $(13,8 \%)$ machten keine eindeutige Angabe. Als Einwand wurde häufig genannt, dass die Kinder erst zwei bis fünf Jahre alt und außerdem mental retardiert seien und es daher schwierig sei, sie aufzuklären und ihre Zustimmung einzuholen (Vorsitzende 7, 8, 13, 19, $22,24,29)$.

\section{Verhalten bei Abwehr (Frage 3)}

Abbildung 5.8 zeigt die Antworten der Vorsitzenden, die die o.g. Studie befürworteten (100\%, N=29), auf die Frage, ob für sie ein eindeutig abwehrendes Verhalten des Kindes ein Ausschluss- bzw. Abbruchkriterium darstellen würde.

19 Vorsitzende $(65,5 \%)$ äußerten, dass ein abwehrendes Verhalten des Kindes für sie ein Ausschluss- oder Abbruchkriterium darstelle. Fünf Vorsitzende (17,2\%) möchten dies

${ }^{39}$ Hierunter werden im Folgenden sowohl die Vorsitzenden gezählt, die die Studie ohne Einschränkungen befürworteten, als auch die Vorsitzenden, die ihr positives Votum an Bedingungen knüpfen würden. 
Fragen 2, 3 und 4
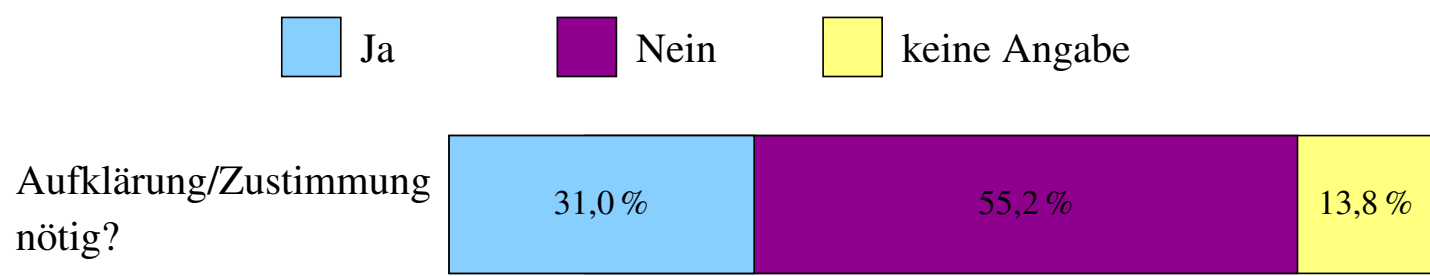

Abbruch/

Ausschluss bei Abwehr?

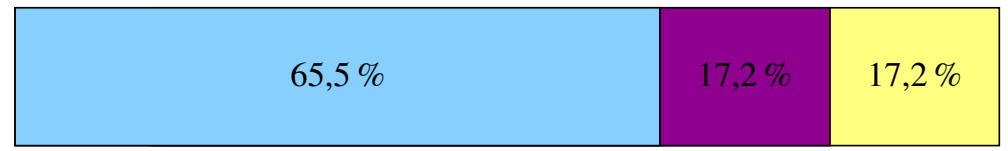

Aufwandsentschädigung nötig?

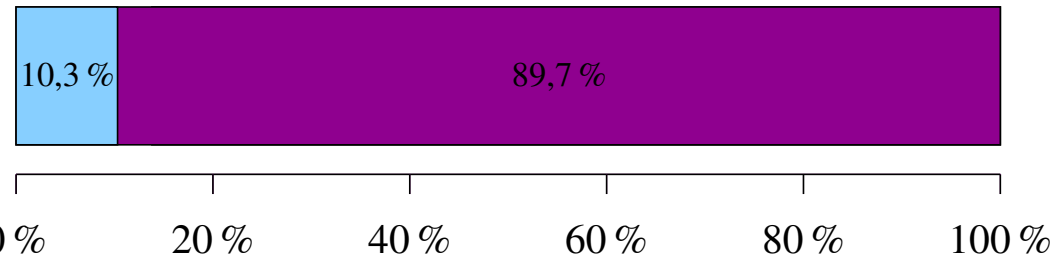

Anteil der Vorsitzenden ( $\mathrm{N}=29)$

Abbildung 5.8: Vignette 1, Fragen 2, 3 und 4: (Vorsitzende, die die Studie befürworteten)

nicht gesondert berücksichtigt sehen. Weitere fünf Vorsitzende $(17,2 \%)$ äußerten sich nicht zu dieser Fragestellung.

\section{Aufwandsentschädigung (Frage 4)}

Abbildung 5.8 zeigt die Antworten der Vorsitzenden, die die o.g. Studie befürworteten $(100 \%, N=29)$, auf die Frage, ob sie eine Aufwandsentschädigung für das Kind für notwendig hielten.

Drei Vorsitzende (10,3\%) hielten eine Aufwandsentschädigung für notwendig. 26 Vorsitzende $(89,7 \%)$ befürworteten eine Entschädigung nicht.

\subsubsection{Vignette 2: Psychokognitive Tests bei HKS und LRS}

\section{Aufklärung/Zustimmung (Frage 2)}

Abbildung 5.9 zeigt die Antworten der Vorsitzenden, die die o. g. Studie befürworteten $(100 \%, N=29)$, auf die Frage, ob sie eine individuelle Aufklärung und Zustimmung des Kindes für erforderlich hielten. 
Fragen 2, 3 und 4
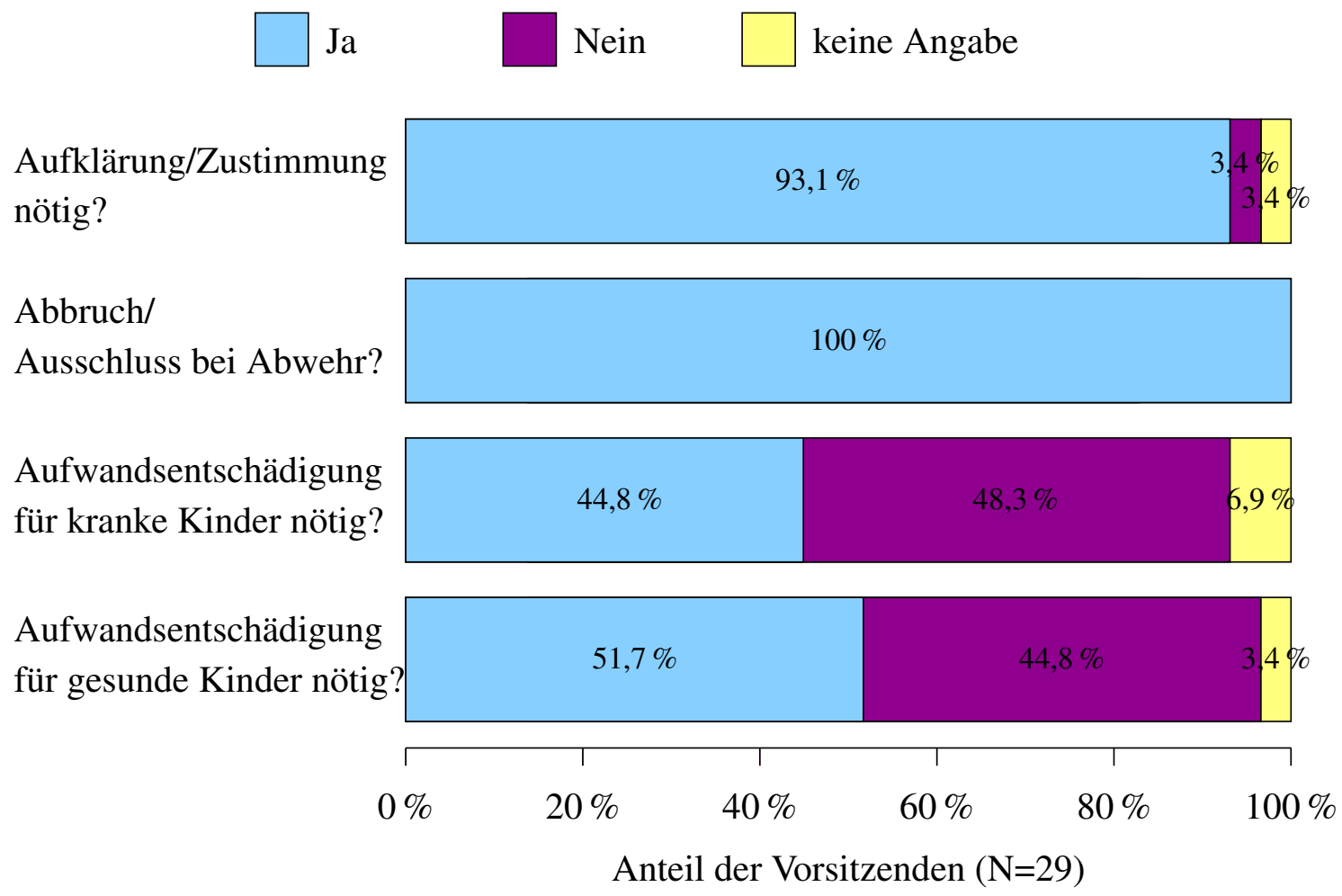

Abbildung 5.9: Vignette 2, Fragen 2, 3 und 4: (Vorsitzende, die die Studie befürworteten) 
27 Vorsitzende $(93,1 \%)$ hielten eine individuelle Aufklärung und Zustimmung des Kindes für erforderlich. Ein Vorsitzender $(3,4 \%)$ sah dafür keine Notwendigkeit, und ein weiterer $(3,4 \%)$ machte keine eindeutige Angabe.

\section{Verhalten bei Abwehr (Frage 3)}

Abbildung 5.9 zeigt die Antworten der Vorsitzenden, die die o.g. Studie befürworteten (100\%, N=29), auf die Frage, ob für sie ein eindeutig abwehrendes Verhalten des Kindes ein Ausschluss- bzw. Abbruchkriterium darstellen würde.

Alle 29 Vorsitzenden (100\%) würden bei einem abwehrenden Verhalten des Kindes einen Abbruch des Versuchs befürworten.

\section{Aufwandsentschädigung (Frage 4)}

Abbildung 5.9 zeigt die Antworten der Vorsitzenden, die die o.g. Studie befürworteten $(100 \%, \mathrm{~N}=29)$, auf die Frage, ob sie eine Aufwandsentschädigung für das Kind für notwendig hielten.

Bei den kranken Kindern plädierten 13 Vorsitzende (44,8\%) dafür und 14 Vorsitzende $(48,3 \%)$ dagegen, während bei den gesunden Kindern 15 Vorsitzende $(51,7 \%)$ im Gegensatz zu 13 Vorsitzenden $(44,8 \%)$ die Notwendigkeit einer Entschädigung sahen. Zwei Vorsitzende $(6,9 \%)$ bzw. ein Vorsitzender $(3,4 \%)$ beantworteten diese Frage nicht. Ein Vorsitzender führte an, dass die Anerkennung für die Studienteilnahme nicht nur durch Geld, sondern auch anders kindgerecht ausgedrückt werden könne (Vorsitzender 7).

\subsubsection{Vignette 3: Orale Placebogabe mit Verzicht auf Standardtherapie bei allergischem Schnupfen}

\section{Aufklärung/Zustimmung (Frage 2)}

Abbildung 5.10 zeigt die Antworten der Vorsitzenden, die die o. g. Studie befürworteten $(65,5 \%, \mathrm{~N}=19)$, auf die Frage, ob sie eine individuelle Aufklärung und Zustimmung des Kindes für erforderlich hielten.

17 Vorsitzende $(89,5 \%)$ hielten eine Aufklärung und Zustimmung der Kinder und Jugendlichen für erforderlich. Zwei Vorsitzende (10,5\%) enthielten sich der Stimme.

\section{Verhalten bei Abwehr (Frage 3)}

Abbildung 5.10 zeigt die Antworten der Vorsitzenden, die die o. g. Studie befürworteten $(65,5 \%, N=19)$, auf die Frage, ob für sie ein eindeutig abwehrendes Verhalten des Kindes ein Ausschluss- bzw. Abbruchkriterium darstellen würde. 
Fragen 2, 3 und 4
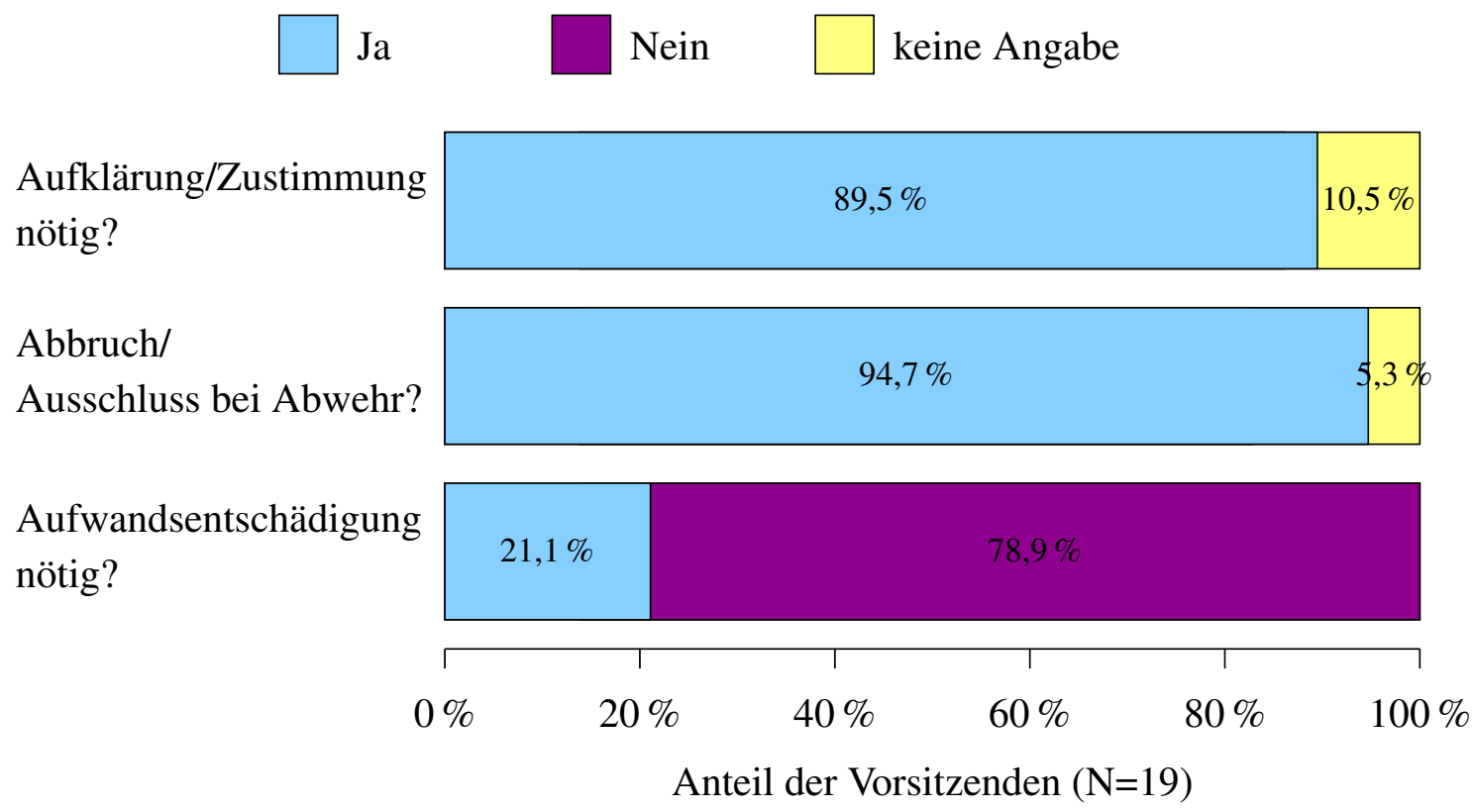

Abbildung 5.10: Vignette 3, Fragen 2, 3 und 4: (Vorsitzende, die die Studie befürworteten)

In einem solchen Fall würden 18 Vorsitzende $(94,7 \%)$ das Kind oder den Jugendlichen nicht in die Studie einbeziehen. Ein Vorsitzender (5,3\%), beantwortete diese Frage nicht.

\section{Aufwandsentschädigung (Frage 4)}

Abbildung 5.10 zeigt die Antworten der Vorsitzenden, die die o.g. Studie befürworteten $(65,5 \%, \mathrm{~N}=19)$, auf die Frage, ob sie eine Aufwandsentschädigung für das Kind für notwendig hielten.

Vier Vorsitzende $(21,1 \%)$ plädierten für eine Aufwandsentschädigung. 15 Vorsitzende $(78,9 \%)$ sprachen sich dagegen aus.

\subsubsection{Vignette 4: Herzmuskelbiopsie bei ohnehin notwendiger Herzoperation}

Abbildung 5.11 zeigt die Antworten der Vorsitzenden, die die o.g. Studie befürworteten $(51,7 \%, \mathrm{~N}=15)$, auf die Frage, ob sie eine Aufwandsentschädigung für das Kind für notwendig hielten. 


\section{Frage 4}

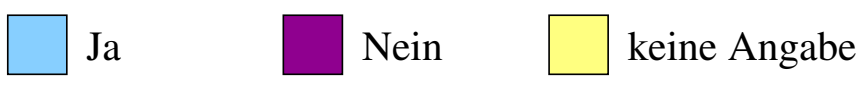

Aufwandsentschädigung nötig?

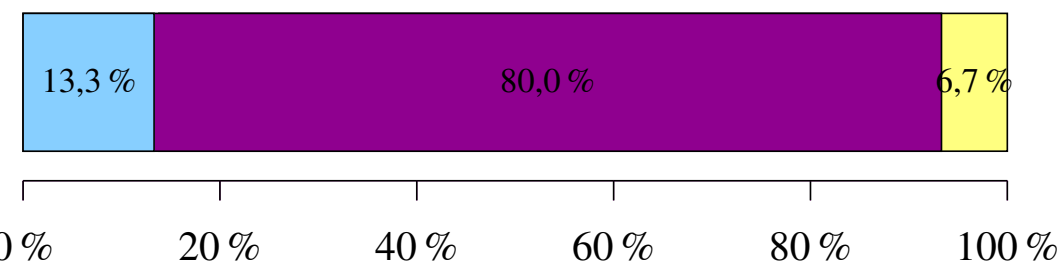

Anteil der Vorsitzenden ( $\mathrm{N}=15)$

Abbildung 5.11: Vignette 4, Frage 4: (Vorsitzende, die die Studie befürworteten)

Zwei Vorsitzende (13,3\%) hielten eine Aufwandsentschädigung für notwendig, zwölf Vorsitzende $(80 \%)$ für nicht nötig. Ein Vorsitzender $(6,7 \%)$ antwortete nicht auf die Frage.

\subsubsection{Vignette 5: Intramuskuläre Placeboinjektionen bei angeborenem Herzfehler}

\section{Aufklärung/Zustimmung (Frage 2)}

Abbildung 5.12 zeigt die Antworten der Vorsitzenden, die die o.g. Studie befürworteten (48,3\%, N=14), auf die Frage, ob sie eine individuelle Aufklärung und Zustimmung des Kindes für erforderlich hielten.

Sechs dieser Vorsitzenden $(42,9 \%)$ hielten eine individuelle Aufklärung und Zustimmung des Kindes für nötig. Vier Vorsitzende $(28,6 \%)$ waren der gegenteiligen Ansicht, ohne diese zu begründen, und vier weitere Vorsitzende $(28,6 \%)$ beantworteten die Frage nicht. Ein Vorsitzender, der alle sechs vorgestellten Forschungsszenarien ohne Einschränkungen für zulässig hielt, formulierte zu dieser Frage die Anmerkung

„Aufklärung ja, Zustimmung nein“ (Vorsitzender 30).

\section{Verhalten bei Abwehr (Frage 3)}

Abbildung 5.12 zeigt die Antworten der Vorsitzenden, die die o.g. Studie befürworteten (48,3\%, N=14), auf die Frage, ob für sie ein eindeutig abwehrendes Verhalten des Kindes ein Ausschluss- bzw. Abbruchkriterium darstellen würde. 
Fragen 2, 3 und 4
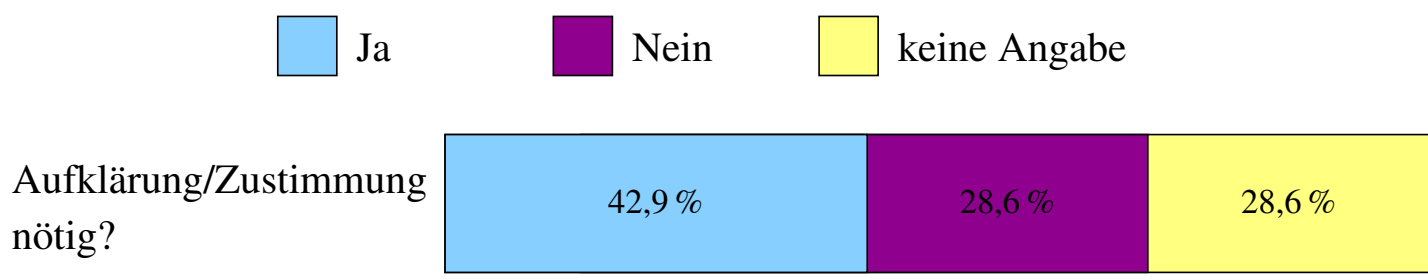

Abbruch/

Ausschluss bei Abwehr?

$78,6 \%$

$42,9 \%$ nötig?

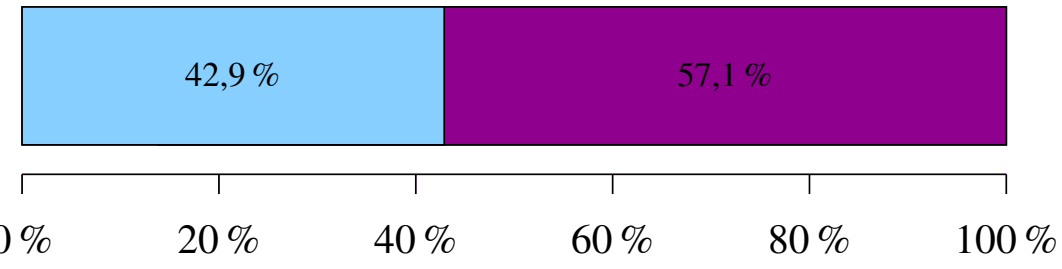

Anteil der Vorsitzenden $(\mathrm{N}=14)$

Abbildung 5.12: Vignette 5, Fragen 2, 3 und 4: (Vorsitzende, die die Studie befürworteten)

Ein ablehnendes Verhalten des Kindes wäre für elf Vorsitzende (78,6\%) ein Ausschlussoder Abbruchkriterium. Zwei Vorsitzende (14,3\%) sprachen sich mit ihren Voten ohne Begründung dagegen aus und ein Vorsitzender $(7,1 \%)$ äußerte sich nicht zu der Frage.

\section{Aufwandsentschädigung (Frage 4)}

Abbildung 5.12 zeigt die Antworten der Vorsitzenden, die die o.g. Studie befürworteten $(48,2 \%, N=14)$, auf die Frage, ob sie eine Aufwandsentschädigung für das Kind für notwendig hielten.

Sechs Vorsitzende $(42,9 \%)$ sahen die Notwendigkeit, die Kinder für ihre Studienteilnahme zu entschädigen. Acht Vorsitzende $(57,1 \%)$ befürworteten eine Aufwandsentschädigung nicht.

\subsubsection{Vignette 6: Zusätzliche Knochenmarkpunktionen bei ALL}

\section{Aufklärung/Zustimmung (Frage 2)}

Abbildung 5.13 zeigt die Antworten der Vorsitzenden, die die o.g. Studie befürworteten $(41,4 \%, \mathrm{~N}=12)$, auf die Frage, ob sie eine individuelle Aufklärung und Zustimmung des 
Kindes für erforderlich hielten. Elf dieser Vorsitzenden (91,7\%) hielten eine Aufklärung und Zustimmung des Kindes zum geplanten Versuch für unerlässlich. Ein Vorsitzender $(8,3 \%)$ beantwortete diese Frage nicht.

Fragen 2, 3 und 4
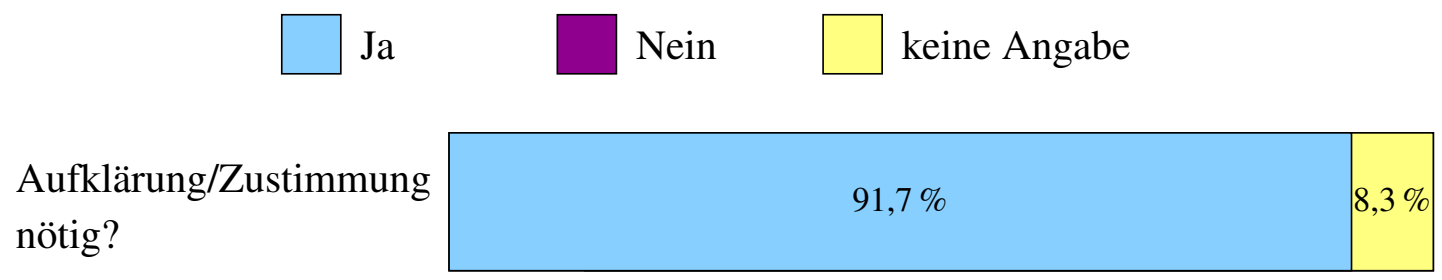

Abbruch/

Ausschluss bei Abwehr?

$91,7 \%$

$50,0 \%$

Aufwandsentschädigung nötig?

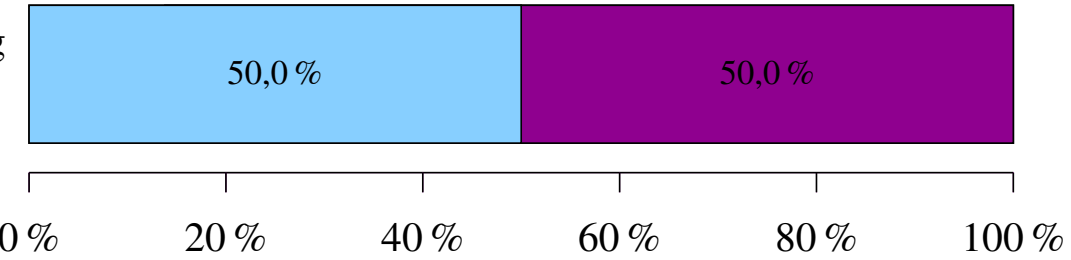

Anteil der Vorsitzenden ( $\mathrm{N}=12)$

Abbildung 5.13: Vignette 6, Fragen 2, 3 und 4: (Vorsitzende, die die Studie befürworteten)

\section{Verhalten bei Abwehr (Frage 3)}

Abbildung 5.13 zeigt die Antworten der Vorsitzenden, die die o.g. Studie befürworteten $(41,4 \%, N=12)$, auf die Frage, ob für sie ein eindeutig abwehrendes Verhalten des Kindes ein Ausschluss- bzw. Abbruchkriterium darstellen würde.

Im Fall eines ablehnenden Verhaltens des Kindes würden elf Vorsitzende (91,7\%) auf die Durchführung des geplanten Eingriffs verzichten. Ein Vorsitzender (8,3\%) machte zu dieser Fragestellung keine Angaben.

\section{Aufwandsentschädigung (Frage 4)}

Abbildung 5.13 zeigt die Antworten der Vorsitzenden, die die o. g. Studie befürworteten $(41,4 \%, \mathrm{~N}=12)$, auf die Frage, ob sie eine Aufwandsentschädigung für das Kind für notwendig hielten.

Sechs Vorsitzende (50,0\%) würden eine Entschädigung befürworten und sechs Vorsitzende $(50,0 \%)$ entschieden sich dagegen. 


\subsubsection{Zusammenfassung der Auswertung zur Beteiligung des Minderjährigen an Entscheidungen über seine Versuchsteilnahme}

Abbildung 5.14 zeigt die Übersicht der Antworten der Vorsitzenden, die die jeweilige Studie befürworteten, auf die Frage, ob für deren Durchführung eine individuelle Aufklärung und Zustimmung des einzelnen Kindes erforderlich sei.

Übersicht: Aufklärung/Zustimmung nötig?

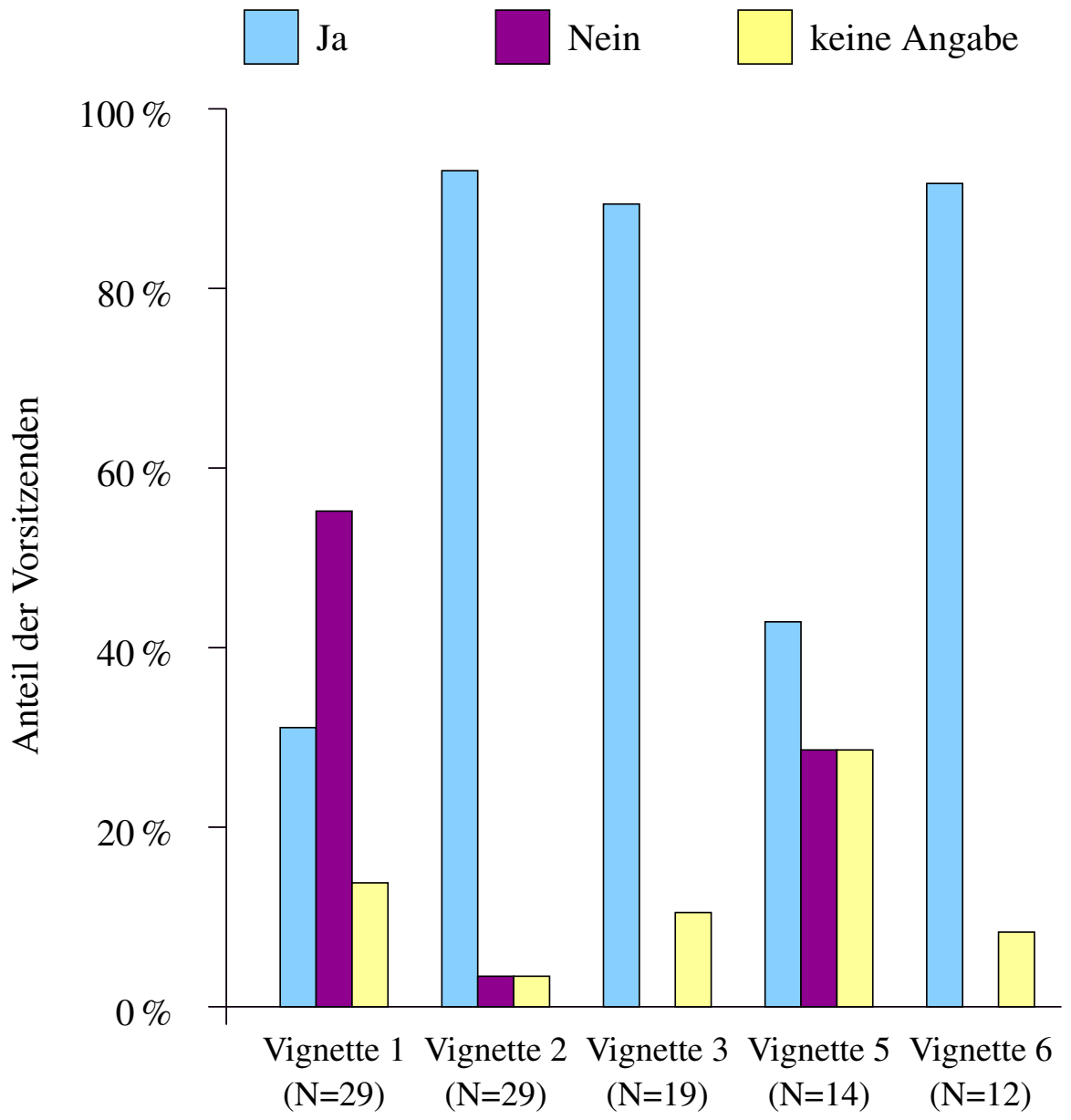

Abbildung 5.14: Übersicht Frage 2: „Halten Sie eine individuelle Aufklärung und Zustimmung des Kindes für erforderlich?“ (Vorsitzende, die die Studie befürworteten)

Abbildung 5.15 zeigt die Übersicht der Antworten der Vorsitzenden, die die jeweilige Studie befürworteten, auf die Frage, ob für sie ein eindeutig abwehrendes Verhalten des Kindes ein Ausschluss- bzw. Abbruchkriterium darstellen würde. 
Übersicht: Abbruch/Ausschluss bei Abwehr?

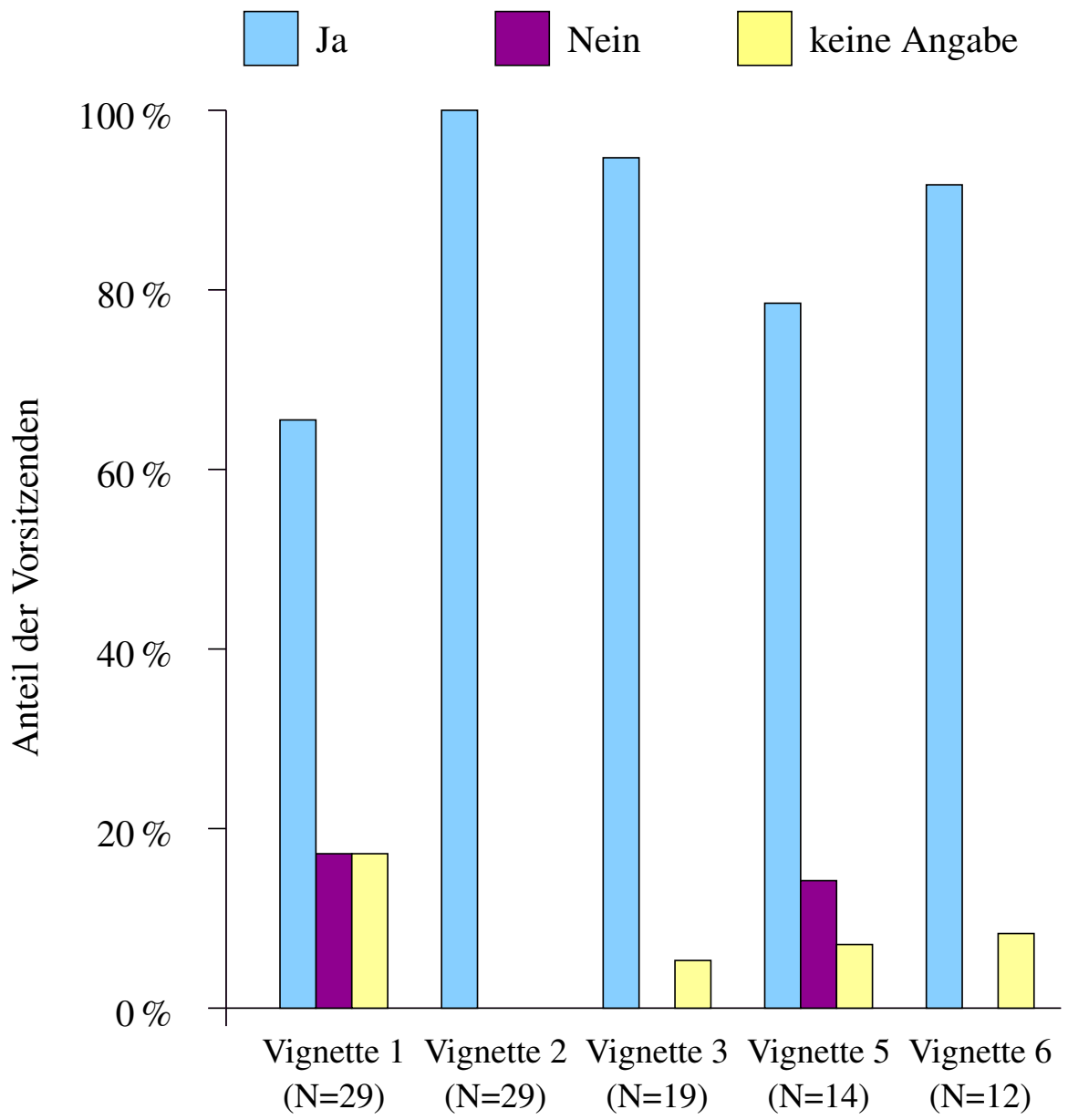

Abbildung 5.15: Übersicht Frage 3: „Wäre für Sie ein eindeutig abwehrendes Verhalten des Kindes ein Ausschluss- bzw. Abbruchkriterium?" (Vorsitzende, die die Studie befürworteten) 
Abbildung 5.16 zeigt die Antworten der Vorsitzenden, die die jeweilige Studie befürworteten, auf die Frage, ob sie eine Aufwandsentschädigung für kranke Kinder (Vignette 1, 3, 5 und 6), kranke und gesunde Kinder (Vignette 2a und 2b) sowie für Eltern (Vignette 4) für notwendig hielten.

Übersicht: Aufwandsentschädigung nötig?
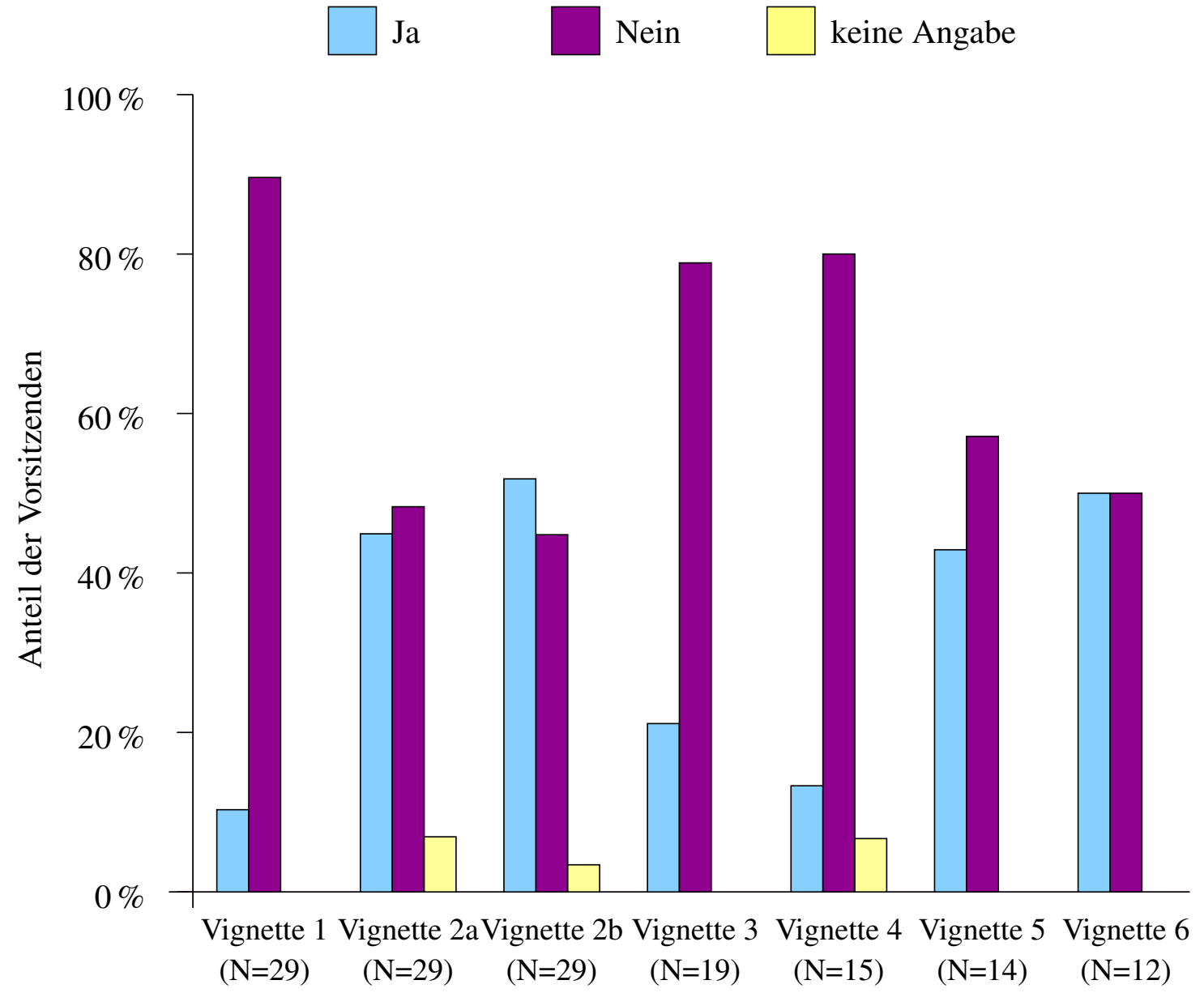

Abbildung 5.16: Übersicht Frage 4: „Halten Sie eine Aufwandsentschädigung für das Kind (Vignette 4: für die Eltern) für notwendig?“ (Vorsitzende, die die Studie befürworteten)

Die Mehrheit der Vorsitzenden, die der Durchführung von der in Vignette 1: Venöse Blutentnahme ohne Notwendigkeit zusätzlicher Punktion bei Rett-Syndrom vorgestellten Studie (keine AMG-Studie, enge Gruppennützigkeit, minimales Risiko, minimale Belastung, zwei- bis fünfjährige, mental retardierte Kinder) zustimmten, erachtete eine individuelle Aufklärung und Zustimmung des jeweiligen Kindes nicht für nötig. Zwei Vorsitzende begründeten diese Entscheidung mit dem geringen Alter der Kinder und ihrer 
mentalen Retardierung. Ein abwehrendes Verhalten würde aber für knapp zwei Drittel der Vorsitzenden ein Abbruch- oder Ausschlusskriterium darstellen. Der überwiegende Teil der Vorsitzenden entschied sich gegen eine Aufwandsentschädigung.

Bis auf einen Vorsitzenden und einen Vorsitzenden, der die Frage nicht beantwortete, hielten alle teilnehmenden Vorsitzenden, die dem Forschungsprojekt in Vignette 2: Psychokognitive Tests bei HKS und LRS (keine AMG-Studie, erweiterte und enge Gruppennützigkeit, minimales Risiko, minimale Belastung, sechs- bis elfjährige Kinder) zustimmten, eine individuelle Aufklärung und Zustimmung des jeweiligen Kindes für nötig. Dass die Vorsitzenden großen Wert auf eine kindgerechte Aufklärung der teilnehmenden Minderjährigen legten, zeigten auch die Anmerkungen zu dieser Vignette (siehe Abschnitt 5.2.2.2). Alle Vorsitzenden sprachen sich für einen Abbruch des Versuchs im Fall eines abwehrenden Verhaltens aus. Etwa die Hälfte der Vorsitzenden erachtete eine Aufwandsentschädigung für sinnvoll, die andere Hälfte für nicht nötig. Zwei Vorsitzende wollten nur die gesunden Kinder für ihre Studienteilnahme entschädigen.

Alle Vorsitzenden, die dem Forschungsprojekt in Vignette 3: Orale Placebogabe mit Verzicht auf Standardtherapie bei allergischem Schnupfen (AMG-Studie, enge Gruppennützigkeit, mehr als minimales Risiko, mehr als minimale Belastung, zwölf- bis 18-jährige Kinder/Jugendliche, Randomisierung und Placebogabe mit Verzicht auf unzuverlässige Standardtherapie) zustimmten, sprachen sich für eine individuelle Aufklärung und Zustimmung des jeweiligen Kindes sowie für einen Abbruch des Versuchs im Fall eines abwehrenden Verhaltens aus bzw. beantworteten vereinzelt diese Frage nicht. Der überwiegende Teil der Vorsitzenden hielt es nicht für notwendig, die Kinder und Jugendlichen für ihre Studienteilnahme zu entschädigen.

Der überwiegende Teil der Vorsitzenden, die dem in Vignette 4 beschriebenen Forschungsprojekt Herzmuskelbiopsie bei ohnehin notwendiger Herzoperation (anteilig AMG-Studie, enge Gruppennützigkeit, mehr als minimales Risiko, minimale Belastung, zwei Wochen bis drei Monate alte Babys, Randomisierung in Studien- und Kontrollgruppe) zustimmten, sprach sich dagegen aus, die jeweiligen Eltern für eine Studienteilnahme ihres Babys zu entschädigen.

Etwa die Hälfte der Vorsitzenden, die dem in Vignette 5 dargestellten Forschungsprojekt Intramuskuläre Placeboinjektionen bei angeborenem Herzfehler (AMG-Studie, enge Gruppennützigkeit, mehr als minimales Risiko, mehr als minimale Belastung, zwei- bis fünfjährige Kinder, Randomisierung und invasive Placebogabe) zustimmten, hielt eine individuelle Aufklärung und Zustimmung des jeweiligen Kindes für nötig. Die Mehrzahl der Vorsitzenden knüpfte ihre Zustimmung für die Studie an die Zustimmung des Minderjährigen zu seiner Studienteilnahme und sprach sich für einen Abbruch des Versuchs im Fall eines abwehrenden Verhaltens aus. Zwei Vorsitzende teilen diese Ansicht nicht. Etwa die Hälfte der in diese Auswertungen eingehenden Vorsitzenden hielt es für notwendig, die Kinder und Jugendlichen für ihre Studienteilnahme zu entschädigen. 
Bis auf einen Vorsitzenden, der die Frage nicht beantwortete, hielten alle teilnehmenden Vorsitzenden, die der in Vignette 6 vorgestellten Studie Zusätzliche Knochenmarkpunktionen bei ALL (AMG-Studie, enge Gruppennützigkeit, mehr als minimales Risiko, mehr als minimale Belastung, sechs- bis zehnjährige, leukämiekranke Kinder) zustimmten, eine individuelle Aufklärung und Zustimmung des jeweiligen Kindes für nötig. Genauso sah das Antwortspektrum zu der Frage nach einem Abbruch des Versuchs im Fall eines abwehrenden Verhaltens aus. Die Hälfte der Vorsitzenden erachtete eine Aufwandsentschädigung für sinnvoll, die andere Hälfte für nicht nötig.

Die Übersicht in Abbildung 5.14 zur Frage nach der Notwendigkeit einer individuellen Aufklärung und Zustimmung des einzelnen Kindes zeigt, dass bei Vignette 5: Intramuskuläre Placeboinjektionen bei angeborenem Herzfehler, einer Studie an zwei- bis fünfjährigen Klein- und Vorschulkindern, die Zulässigkeit der Untersuchung nicht von allen Vorsitzenden an diese Bedingung geknüpft wurde. Bei Vignette 1: Venöse Blutentnahme ohne Notwendigkeit zusätzlicher Punktion bei Rett-Syndrom, in der die Studienkinder mental retardiert waren, stellte sich dieser Punkt noch deutlicher dar: Der überwiegende Teil der Vorsitzenden hielt es nicht für nötig, die an der Studie teilnehmenden Kinder aufzuklären und ihre Zustimmung einzuholen.

Bei den übrigen Vignetten legten, bis auf einen einzelnen Vorsitzenden bei Vignette 2, der seine Entscheidung nicht begründete, alle Vorsitzenden, die die jeweilige Vignette positiv beurteilten, Wert auf eine individuelle Aufklärung und Zustimmung des Kindes oder beantworteten die jeweilige Frage nicht.

Abbildung 5.15 zeigt, dass für die überwiegende Zahl der Vorsitzenden ein abwehrendes Verhalten des Kindes bei allen Forschungsszenarien ein Ausschluss- bzw. Abbruchkriterium wäre. Wie schon bei der Frage nach der Notwendigkeit von Aufklärung und Zustimmung sprachen sich aber bei Vignette 1: Venöse Blutentnahme ohne Notwendigkeit zusätzlicher Punktion bei Rett-Syndrom und Vignette 5: Intramuskuläre Placeboinjektionen bei angeborenem Herzfehler einige Vorsitzende, die die Studien für vertretbar hielten, gegen ein solches Vorgehen aus. Begründet wurde diese Entscheidung zum Teil mit dem geringen Alter der Kinder von jeweils zwei bis fünf Jahren. Dabei spielt der Grad von Risiko und Belastung, der bei Vignette 1 minimal und bei Vignette $5 \mathrm{mehr}$ als minimal ist, anscheinend keine Rolle.

Abbildung 5.16 macht deutlich, dass die Vorsitzenden der deutschen Ethikkommissionen die Zahlung einer Aufwandsentschädigung tendenziell nicht befürworteten. Bei Vignette 2: Psychokognitive Tests bei HKS und LRS und Vignette 6: Zusätzliche Knochenmarkpunktionen bei ALL plädierte immerhin jeweils etwa die Hälfte der Vorsitzenden für eine Aufwandsentschädigung. Gründe für diese Entscheidung wurden nicht genannt. 


\section{Diskussion}

\subsection{Forschung an Kindern und Jugendlichen}

An pädiatrische Medikamente werden spezielle pharmakologische Anforderungen gestellt. Aufgrund von Unterschieden bei der Verstoffwechselung können Wirkstoffdosen aus der Therapie von Erwachsenen nicht einfach dem Körpergewicht eines Minderjährigen angepasst werden, sondern müssen dem jeweiligen Entwicklungsstadium der Kinder und Jugendlichen entsprechen.

Die Situation in der pädiatrischen Pharmakotherapie ist jedoch derzeit nicht zufriedenstellend. Insbesondere schwer kranke Kinder und Jugendliche werden oft mit nicht zugelassenen oder off-label verwendeten Medikamenten behandelt. Ursache ist die nicht ausreichende Zahl aussagekräftiger pharmakologischer Studien an Minderjährigen. Pädiater haben diese Situation oft als bedenklich kritisiert.

Notwendig sind in der Kinderheilkunde und Jugendmedizin und in der Kinder- und Jugendpsychiatrie zudem Studien zur Krankheitsursachenforschung und zur Erhebung von Normalwerten, insbesondere für die Beurteilung der unauffälligen kindlichen Entwicklung. Forschung dieser Art strebt meistens keinen direkten Eigennutzen für den minderjährigen Versuchsteilnehmer an. Sie wird aber später voraussichtlich Kindern und Jugendlichen der gleichen Krankheits- oder Altersgruppe nützen und hat daher einen ausschließlich gruppennützigen Ansatz. Teilweise muss sie an gesunden Minderjährigen erfolgen, um aussagekräftig zu sein. Auch die von Pädiatern oft eingeforderte Medikamentenforschung ist gelegentlich ohne unmittelbaren direkten Eigennutzen für die Versuchsperson: Beispiele dafür sind die Randomisierung eines Studienteilnehmers in eine Placebogruppe oder in eine Kontrollgruppe ohne Therapie.

Eine Ursache für die nach wie vor geringe Zahl von Studien an Kindern und Jugendlichen ist u. a. in den damit verbundenen ethischen Konflikten zu suchen. Kinder und Jugendliche bilden eine so genannte vulnerable Gruppe, die besonders geschützt werden muss, wenn sie in medizinische Forschung einbezogen werden soll. Insbesondere die gruppennützige Forschung an Kindern und Jugendlichen, die keinen direkten Nutzen für die minderjährigen Versuchspersonen anstrebt, benötigt besondere Rechtfertigung. Weiterhin muss aus ethischer Perspektive geklärt werden, ab welchem Alter oder Reifegrad und in welcher 
Form ein Minderjähriger an Entscheidungen über seine Versuchsteilnahme beteiligt werden soll.

\subsection{Risiko und Belastung}

In Gesetzen und Empfehlungen wird gruppennützige Forschung an Kindern und Jugendlichen daher üblicherweise gesondert geregelt. Ihre Zulässigkeit wird insbesondere vom Risiko und von der Belastung der geplanten Eingriffe abhängig gemacht, wobei die jeweils aufgestellten Bedingungen im Detail variieren.

Das deutsche Medizinproduktegesetz (MPG 2002) ist nicht eindeutig bezüglich seiner Regelung gruppennütziger Forschung an Kindern und Jugendlichen. Es entspricht im Wortlaut nahezu vollständig dem alten Arzneimittelgesetz (AMG 1994), das von Juristen in dieser Form divergierend interpretiert (Fröhlich 1999, Taupitz 1999, Wachenhausen 2000) und von mehreren Wissenschaftler als nicht eindeutig kritisiert worden war (Dahl und Wiesemann 2001, Pestalozza 2004, Vollmann 2000). Ein Maß für das zulässige Risiko bzw. die zulässige Belastung dieses Typs von Forschung wird im MPG nicht angegeben.

Als Grenze für das zulässige Risiko und die zulässige Belastung bei gruppennütziger Forschung an Kindern und Jugendlichen werden in der 12. Novelle des Arzneimittelgesetzes von 2004 das minimale Risiko und die minimale Belastung genannt. Allerdings eröffnet die Definition des minimalen Risikos als ein Risiko, das ,allenfalls zu einer sehr geringfügigen und vorübergehenden Beeinträchtigung der Gesundheit der betroffenen Person“ führt (AMG 2004, § 41 (2) 2.d) und der minimalen Belastung als „Unannehmlichkeiten“, welche ,für die betroffene Person allenfalls vorübergehend auftreten und sehr geringfügig sein werden“ (AMG 2004, § 41 (2) 2.d), einen Interpretationsspielraum. Welchen Zeitraum umfasst der Ausdruck ,,allenfalls vorübergehend“? Wie ist eine ,sehr geringfügige“ Beeinträchtigung der Gesundheit oder eine ,sehr geringfügige“ Unannehmlichkeit einzugrenzen? ${ }^{40}$

Darüber hinaus sind einige wichtige Fragen und Bereiche der gruppennützigen Forschung an Kindern und Jugendlichen in deutschen Gesetzen nicht geregelt: Placeboforschung als ein häufiger Spezialfall von Arzneimittelstudien findet im AMG keine Erwähnung. Zudem gibt es in Deutschland keine rechtliche Regelung von Forschung, die weder Arzneimittel noch Medizinprodukte betrifft.

\footnotetext{
${ }^{40}$ In diesem Zusammenhang sei an die in Abschnitt 2.3.2 beschriebene Untersuchung von Jeffrey Janofsky und Barbara Starfield erinnert, die zeigte, wie sehr das Verständnis von minimalem Risiko bei amerikanischen Pädiatern trotz entsprechender Gesetzestexte variierte (Janofsky und Starfield 1981).
} 
Auf diese Bereiche geht jedoch die Zentrale Kommission zur Wahrung ethischer Grundsätze in der Medizin und ihren Grenzgebieten in einer Stellungnahme von 2004 ein (Stellungnahme der ZEKO 2004). Sie gibt Empfehlungen für den Umgang mit gruppennütziger Forschung einschließlich der Placeboforschung und solcher Studien, die nicht Arzneimittel oder Medizinprodukte betreffen. Im Gegensatz zum AMG können laut Stellungnahme der ZEKO auch bei gruppennütziger Forschung an Kindern und Jugendlichen ein mehr als minimales Risiko sowie eine mehr als minimale Belastung zulässig sein. Die Begriffe werden für diesen Zweck präzise eingegrenzt. Die Regelung von gruppennütziger Forschung wird jedoch in Teilen als zu großzügig kritisiert (Wiesemann 2005). Der Stellungnahme fehlt zudem die rechtliche Verbindlichkeit.

Auf internationaler Ebene werden weitere Varianten der Eingrenzung des zulässigen Risikos und der zulässigen Belastung von gruppennütziger Forschung an Minderjährigen verwendet, die in der vorliegenden Arbeit im Detail dargestellt wurden. Ihre Heterogenität kann zu einer weiteren Verunsicherung hinsichtlich der Bewertung gruppennütziger Forschung in der Pädiatrie beitragen.

Die Umsetzung der in Gesetzen und Empfehlungen festgelegten Bedingungen für gruppennützige Forschung an Kindern und Jugendlichen ist in allen Ländern der Europäischen Union insbesondere Aufgabe der Ethikkommissionen zur Forschung am Menschen, die geplante Forschungsprojekte begutachten. Sie beurteilen den Prüfplan eines Antragstellers, der u. a. Angaben zum voraussichtlichen Ablauf der Studie und zu geplanten Interventionen sowie die schriftlichen Aufklärungs- und Einwilligungsformulare für Eltern bzw. gesetzliche Vertreter und ggf. für die beteiligten Kinder und Jugendlichen enthält. Anschließend entscheiden sie über die Zulässigkeit des Forschungsprojektes bzw. geben Anweisungen zu dessen Änderung. Die rechtlichen und standesethischen Regelungen, auf die sie sich dabei stützen müssen, bieten ihnen dabei nicht immer klare Anhaltspunkte.

Die vorliegende Vignettenstudie entstand aus der Frage, was diese Situation für Ethikkommissionen bedeutet. Insbesondere sollte erforscht werden, ob und wie sich Ethikkommissionen bei ihren Entscheidungen zu gruppennütziger Forschung an Kindern und Jugendlichen am Grad von Risiko und Belastung orientieren und welche Bedeutung für sie die Einstellung eines Minderjährigen zu seiner Studienteilnahme hat. Die Studie ist die erste bundesweit durchgeführte Arbeit, die die Einstellung von Vorsitzenden deutscher Ethikkommissionen zu gruppennütziger Forschung an Kindern und Jugendlichen empirisch untersucht.

Zur Bearbeitung der Fragen wurde ein Fragebogen mit sechs Fallvignetten auf der Basis authentischer Studienentwürfe konzipiert und an die Vorsitzenden sämtlicher deutscher Ethikkommissionen gesandt. Die Antwortquote von 59,2\% zeigt, dass das Thema auf das Interesse der Vorsitzenden stieß. Diese verfügten in der weitaus überwiegenden Zahl über umfangreiche Erfahrung bei der Begutachtung von Studienprojekten (siehe Tabelle 5.2). 
Um sowohl die Realitätstreue der Fallvignetten als auch die Häufigkeit von gruppennütziger Forschung mit mehr als minimalem Risiko und mehr als minimaler Belastung an Minderjährigen am Beispiel eines bundesdeutschen Universitätsklinikums zu überprüfen, wurden parallel zu der Befragung der Vorsitzenden sämtliche Anträge zur Forschung an Kindern und Jugendlichen an die Ethikkommission Göttingen aus den Jahren 1999 bis 2003 ausgewertet. Der überwiegende Anteil dieser Anträge (57 Anträge, 78,1\%) hatte einen gruppennützigen Ansatz, das heißt, die Studie strebte einen engen bzw. erweiterten Gruppennutzen an (siehe Abschnitt 4.2.1).

Die Auswertung dieser Anträge zeigt auch, dass Eingriffe mit mehr als minimalem Risiko und mehr als minimaler Belastung nicht nur in der Theorie vorkommen. 19 von 57 geplanten gruppennützigen Studien $(33,3 \%)$ sollten Eingriffe mit mehr als minimalem Risiko und 18 Studien (31,6\%) Eingriffe mit mehr als minimaler Belastung beinhalten (siehe Abschnitt 4.2.2). In den meisten Fällen ging es dabei um Venenpunktionen sowie um den Einsatz eines Placebos. Es waren aber auch invasivere Eingriffe zu verzeichnen, wie z. B. intramuskuläre Placeboinjektionen oder eine intravenöse Kontrastmittelgabe.

Ethikkommissionen kommen demnach in der Realität durchaus in die Situation, über gruppennützige Forschungsprojekte mit mehr als minimalem Risiko oder mehr als minimaler Belastung urteilen zu müssen. Dabei sind auch Entscheidungen über die Zulässigkeit von deutlich invasiveren Eingriffen wie intramuskulären Placeboinjektionen erforderlich.

Wie beurteilen Vorsitzende von Ethikkommissionen diese Art der gruppennützigen Forschung an Kindern und Jugendlichen? Die Auswertung der Frage nach der Zulässigkeit von verschiedenen Studientypen (siehe Abschnitt 5.2.2) zeigt, dass die Vorsitzenden Forschung ohne direkten Eigennutzen an Kindern und Jugendlichen grundsätzlich akzeptierten.

Wie wird das zulässige Risiko und die zulässige Belastung bei gruppennütziger Forschung an Kindern und Jugendlichen eingegrenzt? Gruppennützige Forschung mit minimalem Risiko und minimaler Belastung (Vignette 1 und Vignette 2) wurde einstimmig für zulässig gehalten. Den Studientyp mit mehr als minimalem Risiko und minimaler Belastung (Vignette 4) hielt die Mehrheit der Vorsitzenden für vertretbar. Studientypen mit zunehmend invasiven Interventionen mit mehr als minimalem Risiko und mehr als minimaler Belastung (Vignette 3, Vignette 5 und Vignette 6) waren für einen größeren bzw. den überwiegenden Teil der Vorsitzenden nicht zulässig. Sie begründeten ihre Entscheidung gegen eine Studie u. a. mit dem zu hohen Grad an Risiko und Belastung für die teilnehmenden Kinder und Jugendlichen. Allerdings stand über ein Drittel der Vorsitzenden auch diesen Studientypen mit mehr als minimalem Risiko und mehr als minimaler Belastung befürwortend gegenüber.

Gruppennützige Forschung an Kindern und Jugendlichen mit mehr als minimalem Risiko und mehr als minimaler Belastung wurde also nicht grundsätzlich ausgeschlossen. Dies 
betraf sowohl Studien zu Arzneimitteln und Medizinprodukten als auch jene bei der Auswertung aller Anträge zur Forschung an Kindern und Jugendlichen an die Ethikkommission Göttingen der Jahre 1999-2003 keinesfalls seltenen Studien (siehe Abschnitt 4.2.3), die der Krankheitsursachenforschung oder der Erhebung von Vergleichswerten in Normalkollektiven dienten. Über gruppennützige Forschungsprojekte mit mehr als minimalem Risiko und mehr als minimaler Belastung entschieden die Vorsitzenden jedoch nicht einheitlich. Minimales Risiko und minimale Belastung waren für einen Teil von ihnen keine ausschlaggebenden Kriterien bei der Bewertung einer Studie. Dabei ist vor allen Dingen die Heterogenität der Urteile bemerkenswert.

Offenbar legen Vorsitzende von Ethikkommissionen unterschiedliche Kriterien bei der Beurteilung von Studien an. Die sich daraus ergebende Situation ist unbefriedigend, sowohl für in Studien integrierte Kinder und Jugendliche als auch für ihre Eltern bzw. gesetzlichen Vertreter. Den begutachtenden Wissenschaftlern wie auch der Öffentlichkeit fehlt Klarheit darüber, welche Standards zum Schutz minderjähriger Versuchspersonen angewendet werden und woran sich die Beurteilung von Studien an Kindern und Jugendlichen orientieren sollte.

Insbesondere die Begutachtung multizentrischer Studien, an der mehrere lokale Ethikkommissionen beteiligt sind, stellt sich im Hinblick auf diese Ergebnisse als problematisch dar. Zur Verbesserung dieser Situation verabschiedete der Arbeitskreis Medizinischer Ethik-Kommissionen „Empfehlungen zur Begutachtung klinischer Studien durch Ethik-Kommissionen“ (Raspe 2005). Sie sollen die Beratungs- und Begutachtungsprozesse seiner Mitglieder unterstützen (Raspe et al. 2005).

Diese Empfehlungen können in Zukunft hilfreich für Begutachtungsprozesse von Ethikkommissionen sein. Trotzdem bedarf es - gerade im Hinblick auf die in der Vignettenstudie gezeigten Bewertungsprobleme von gruppennützigen Forschungsprojekten mit mehr als minimalem Risiko und mehr als minimaler Belastung - einer präziseren und umfassenderen Regelung der ausschließlich gruppennützigen Forschung an Kindern und Jugendlichen. Die Begriffe Risiko und Belastung sollten inhaltlich präzisiert und konkretisiert werden. Weiterhin sind wichtige Bereiche dieser Forschung mit der 12. AMG-Novelle nicht geklärt, erzeugen aber deutliche Probleme in der Praxis: Forschung mit Einsatz eines Placebos, die ein Drittel der ausgewerteten Arzneimittelstudien ausmachte und in der Vignettenstudie heterogen beurteilt wurde, wird im Gesetz nicht erwähnt. Weiterhin nicht gelöst ist die Frage, wie mit Studien mit mehr als minimalem Risiko und mehr als minimaler Belastung umzugehen ist. Dieser Studientyp umfasste etwa ein Drittel der ausgewerteten Anträge zur Forschung an Kindern und Jugendlichen an die Ethikkommission der Universität Göttingen in den Jahren 1999-2003. Die Mehrheit dieser Anträge betraf außerdem Studien, in deren Rahmen nicht an Arzneimitteln oder Medizinprodukten geforscht werden sollte. Für sie müsste dringend eine rechtlich verbindliche Regelung geschaffen werden. 
Sollten gruppennützige Forschungsprojekte mit mehr als minimalem Risiko und mehr als minimaler Belastung - wie es die ZEKO schon jetzt empfiehlt - in Zukunft nicht grundsätzlich unzulässig sein, aber doch auf Einzelfälle beschränkt bleiben, wäre es wichtig, die einheitliche Beurteilung zu erleichtern und damit zugleich eine größere Kontrolle über diesen international wie national umstrittenen Typ der Forschung an Kindern und Jugendlichen zu erreichen. Denkbar wäre es, diese Studienvorhaben nach der Begutachtung durch eine lokale Ethikkommission an eine überregionale Ethikkommission weiterzuleiten. So wären zumindest Entscheidungen nach einheitlichen Bedingungen über Studien mit mehr als minimalem Risiko und mehr als minimaler Belastung gewährleistet.

\subsection{Beteiligung des Minderjährigen an Entscheidungen über seine Versuchsteilnahme}

Eine weitere Bedingung zur Regelung der Zulässigkeit von gruppennütziger Forschung an Kindern und Jugendlichen ist der informed consent der Eltern bzw. der gesetzlichen Vertreter sowie in unterschiedlichem Ausmaß die Beteiligung des Minderjährigen an Entscheidungen über seine Versuchsteilnahme.

Die Deklaration von Helsinki (Deklaration von Helsinki 2004) und die Guidelines des CIOMS von 2002 verlangen die Einholung der Zustimmung des Minderjährigen vor seiner Versuchsteilnahme. Das MPG von 2002, das alte AMG von 1994 und dessen aktuelle Version von 2004 sowie die Stellungnahme der ZEKO von 2004 fordern die Einwilligung eines Kindes oder Jugendlichen, wenn der Minderjährige Wesen, Bedeutung und Tragweite eines Forschungsprojektes erfassen und seinen Willen danach ausrichten kann.

Zum Vorgehen im Fall eines abwehrenden Verhaltens seitens eines minderjährigen Studienteilnehmers enthalten die Guidelines des CIOMS, die Richtlinie 2001/20/EG, das AMG von 2004 und die Stellungnahme der ZEKO von 2004 variierende Aussagen. Die Richtlinie 2001/20/EG macht die Berücksichtigung einer Ablehnung davon abhängig, ob das Kind oder der Jugendliche ,sich eine eigene Meinung bilden kann und die erhaltenen Informationen zu beurteilen weiß“ (Richtlinie 2001/20/EG 2001, Artikel 4 c). Diese Einschränkung machen die Guidelines des CIOMS von 2002, das AMG und die Stellungnahme der ZEKO von 2004 nicht.

Die Guidelines des CIOMS geben dagegen detaillierte Hinweise zur Eingrenzung eines abwehrenden Verhaltens bei jüngeren Kindern und definieren die deliberate objection als eine von unspezifischen Reaktionen auf Stimuli unterscheidbare Abwehrreaktion, die respektiert werden solle (Guidelines des CIOMS 2002, Commentary on Guideline 14). Das derzeit gültige AMG fordert, dass es beachtet werden müsse, wenn ein Minderjähriger erklärt oder ,,in sonstiger Weise zum Ausdruck“ bringt, nicht an einer Studie teilnehmen zu wollen (AMG 2004, § 40 (4) 3.). Die ZEKO legt fest, dass auch schon unspezifisches 
Abwehrverhalten wie „Irritation, Abwehr, Angst im situativen Kontext“ (Stellungnahme der ZEKO 2004, 4.8) Abbruchkriterium eines Versuchs sein solle.

Die positive Einstellung eines Minderjährigen zu seiner Studienteilnahme war auch für die Mehrzahl der Vorsitzenden der deutschen Ethikkommissionen eine Voraussetzung für deren Durchführung, wenn sie sich bei der Beurteilung der Studie nicht am Grad von Risiko oder Belastung orientierten (siehe Abschnitt 5.2.3). Die Zulässigkeit von gruppennütziger Forschung mit mehr als minimalem Risiko und mehr als minimaler Belastung wurde demnach in der Regel von der befürwortenden Haltung eines Minderjährigen zu seiner Versuchsteilnahme abhängig gemacht.

Eine Ausnahme zeigte sich allerdings bei der Bewertung von Willensäußerungen bei Klein- und Vorschulkindern. Jüngeren Kindern wurden anscheinend differenzierte Willensäußerungen weniger zugetraut als älteren Kindern und Jugendlichen bzw. es wurde als schwierig angesehen, das abwehrende Verhalten eines kleineren Kindes richtig einzuschätzen. Das Gewicht der Einstellung eines Minderjährigen wurde offensichtlich von dessen Alter abhängig gemacht.

Sind deshalb zu dieser Problematik differenziertere Vorgaben in rechtlichen oder ethischen Dokumenten nötig? Die Eingrenzung von abwehrendem Verhalten ist gerade bei jüngeren Kindern nicht einfach. Die Aussagen des aktuellen AMG, der ZEKO und der Guidelines des CIOMS geben sinnvolle Entscheidungshilfen vor. Trotzdem ist eine genauere Definition eines abwehrenden Verhaltens in Gesetzen und Empfehlungen sinnvoll, um ein für die Praxis taugliches Vorgehen zu etablieren. Hier könnten beispielsweise unterschiedliche Definitionen und Beispiele für das praktische Vorgehen in Abhängigkeit von der Altersstufe der Kinder hilfreich sein.

Aufklärung und Zustimmung der Kinder und Jugendlichen sowie Aufklärung und Einwilligung ihrer Eltern bzw. gesetzlichen Vertreter haben anscheinend für die Vorsitzenden der Ethikkommissionen einen großen Stellenwert, besonders, wenn es um Forschungsprojekte mit mehr als minimalem Risiko und mehr als minimaler Belastung geht. Dies zeigen auch die Anmerkungen der Vorsitzenden zu den einzelnen Vignetten: Die Vorsitzenden betonten hier besonders häufig die Notwendigkeit einer sorgfältigen Aufklärung und Einwilligung der Eltern bzw. der gesetzlichen Vertreter (siehe Abschnitt 5.2.2).

Diese Bedingungen sollten Standardbedingungen klinischer Studien sein. Dass es viele Vorsitzende für nötig hielten, gesondert auf sie hinzuweisen, könnte als Hinweis verstanden werden, dass es ihrem Eindruck nach manchmal an der praktischen Umsetzung dieser Grundvoraussetzungen mangelt.

Eine andere Hypothese ist allerdings, dass sich die Vorsitzenden bei der Beurteilung heikler Forschungsprojekte mit mehr als minimalem Risiko und mehr als minimaler Belastung durch die ausführliche Aufklärung der Eltern bzw. gesetzlichen Vertreter absichern wollen. Diese Haltung ist deshalb problematisch, weil es ethisch und rechtlich nicht klar ist, ob eine Stellvertretung bei der Einwilligung in gruppennützige Forschungsprojekte 
durch Eltern oder andere gesetzliche Vertreter gerade bei älteren Kindern und Jugendlichen überhaupt als ausreichend angesehen werden kann. Außerdem wird eine rechtlich problematische Studie auch durch umfassende Aufklärung und nachdrückliche Einwilligung nicht per se legitimiert.

Wenn nur die besonders sorgfältige Aufklärung als Absicherung bei rechtlicher Unsicherheit dient, so erscheint die im vorigen Abschnitt gestellte Forderung nach einer präziseren Regelung des Umgangs mit Studien mit mehr als minimalem Risiko und mehr als minimaler Belastung umso dringender.

Ein weiterer Aspekt der Beteiligung des Minderjährigen an Entscheidungen über seine Versuchsteilnahme ist die Zahlung eines Versuchshonorars oder einer Aufwandsentschädigung bzw. die Gewährung anderer Gegenleistungen. Im negativen Fall kann es sich hierbei um eine Einschränkung der Freiwilligkeit der Versuchsperson handeln. Im positiven Sinne kann aber auch Anerkennung für die Versuchsteilnahme ausgedrückt und eine Entschädigung für den damit verbundenen Aufwand geleistet werden.

Die Richtlinie 2001/20/EG, die Guidelines des CIOMS von 2002 und das AMG von 2004 stellen Bedingungen zur Zulässigkeit der Honorierung einer Versuchsteilnahme. Dabei legt das AMG fest, dass „Vorteile mit Ausnahme einer angemessenen Entschädigung“ nicht gewährt werden dürfen (AMG 2004, § 40 (4) 5.). Die beiden anderen Empfehlungen machen vergleichbare Angaben. Dabei wird nicht differenziert, ob Eltern bzw. gesetzliche Vertreter oder der an einer Studie teilnehmende Minderjährige eine Entschädigung erhalten sollen.

Zur Frage nach einer Aufwandsentschädigung und einer ggf. daraus folgenden Beeinflussung der Freiwilligkeit der Versuchsteilnahme eines Minderjährigen zeigten die Ergebnisse der Vignettenstudie, dass die Vorsitzenden von Ethikkommissionen in der Mehrheit keine Entschädigung für Kinder und Jugendliche bzw. für die Eltern oder andere gesetzliche Vertreter (Vignette 4) forderten (siehe Abschnitt 5.2.3). Ob es den Vorsitzenden dabei darum ging, den Willen eines Minderjährigen oder seiner Eltern bzw. gesetzlichen Vertreter nicht zu beeinflussen, oder ob andere Gründe für den Verzicht auf eine Honorierung vorlagen, ging aus den Antworten nicht hervor.

Die ausgewerteten Anträge an die Ethikkommission Göttingen bestätigen diese Tendenz: Der überwiegende Anteil der Projekte sah keine Aufwandsentschädigung für Kinder und Jugendliche bzw. deren Eltern oder andere gesetzliche Vertreter vor (siehe Abschnitt 4.2.4). Gründe dafür konnten den Anträgen nicht entnommen werden.

In Gesetzen und Empfehlungen gibt es bisher wenig gesonderte Aussagen zur Honorierung einer Versuchsteilnahme von Kindern und Jugendlichen. Vor dem Hintergrund einer potentiellen Beeinflussung der Freiwilligkeit durch eine Aufwandsentschädigung ist es sinnvoll, dieses Thema weitergehend zu diskutieren und ggf. im AMG differenzierter zu formulieren. 


\subsection{Ausblick}

In der vorliegenden Studie wurde nur die Ansicht von Vorsitzenden deutscher Ethikkommissionen erhoben. Die Einstellung der übrigen Mitglieder von Kommissionen zu den zur Diskussion gestellten Forschungsprojekten konnte nicht erfasst werden. Dies sollte ggf. das Ziel einer größer angelegten Studie sein, welche die Auswirkungen des neuen AMG auf die Arbeit von Ethikkommissionen überprüfen könnte.

Die vorliegende Studie hat nicht nach der Einstellung jener Personen gefragt, die Gegenstand der Forschung sind, nämlich nach der Sicht der Kinder und Jugendlichen selbst. Es gibt Untersuchungen zu der Frage, wie Eltern die Studienteilnahme ihrer Kinder erleben (Dahl et al. 2002, Snowdon et al. 1998) und zur Einstellung von Ärzten und Pflegepersonal zur Forschung an Minderjährigen (Dahl et al. 2002). Interviews mit Kindern und Jugendlichen zu ihren Partizipationsbedürfnissen und -möglichkeiten bei Therapieentscheidungen wurden ebenfalls schon durchgeführt (Fegert et al. 2005). Bisher fehlt aber eine Befragung von Minderjährigen zu ihrer Einstellung zur Teilnahme an gruppennütziger Forschung. Die Bewertung dieses Aspektes von Forschung durch Kinder und Jugendliche könnte wichtige Anhaltspunkte für seine ethische Analyse und praktische Regelung bieten. Damit würden Kinder und Jugendliche auch in diesem Punkt ernst genommen, so wie es schon bei der ethischen Diskussion über ihre Beteiligung an Entscheidungen über eine Versuchsteilnahme gefordert wird. Aber auch dies ist ein Anliegen für zukünftige Forschung. 


\section{Zusammenfassung}

In der vorliegenden Arbeit wurde die Einstellung von Vorsitzenden deutscher Ethikkommissionen zu gruppennütziger Forschung an Kindern und Jugendlichen untersucht. Im Einzelnen ging es um die Fragen, in welchem Maß sich die Vorsitzenden bei der Beurteilung solcher Studien an den Begriffen minimales Risiko und minimale Belastung orientieren und welche Bedeutung sie der Beteiligung eines Minderjährigen an Entscheidungen über seine Versuchsteilnahme beimessen.

Eine Auswertung sämtlicher Anträge zur Forschung an Kindern und Jugendlichen in den Jahren 1999-2003 an die Ethikkommission Göttingen zeigte die praktische Relevanz dieser Fragen: Der überwiegende Anteil der Anträge hatte einen gruppennützigen Ansatz, und etwa ein Drittel beschrieb Forschungsprojekte mit mehr als minimalem Risiko und mehr als minimaler Belastung.

Die Vignettenstudie ergab, dass für einen Teil der Vorsitzenden der deutschen Ethikkommissionen minimales Risiko und minimale Belastung keine entscheidenden Bedingungen für die Zulässigkeit von gruppennütziger Forschung an Minderjährigen darstellten. Meist wurde die Befürwortung eines Studienprojektes in einem solchen Fall aber an die positive Einstellung des Minderjährigen zu seiner Studienteilnahme geknüpft. Der Willensäußerung von Klein- und Vorschulkindern wurde allerdings weniger Bedeutung zugemessen als der von älteren Kindern.

Die durch die Ergebnisse der Studie gezeigte sehr heterogene Beurteilung gruppennütziger Forschungsprojekte an Kindern und Jugendlichen ist problematisch. Eine mögliche Ursache dafür ist eine unklare rechtliche Regelung zum Zeitpunkt der Erhebung. Allerdings besteht auch zum aktuellen Zeitpunkt, nach der 12. AMG-Novelle von 2004, der Bedarf einer inhaltlichen Präzisierung und Konkretisierung der Begriffe Risiko und Belastung in deutschen Gesetzen. Weiterhin muss festgelegt werden, wie mit Studien mit mehr als minimalem Risiko und mehr als minimaler Belastung umgegangen werden soll. Zudem fehlt es auch heute noch an einer gesetzlichen Regelung von Forschungsprojekten, die nicht Arzneimittel oder Medizinprodukte betreffen. Diese hatten im Kollektiv der ausgewerteten Anträge an eine deutsche Ethikkommission einen Anteil von mehr als $50 \%$. Die vorliegende Arbeit zeigt, dass eine Verbesserung der rechtlichen Regelung von Forschung an Kindern und Jugendlichen dringend vonnöten ist. 


\section{Anhang}

\subsection{Geplante Eingriffe gruppennütziger Forschungsprojekte}

\begin{tabular}{ll}
\hline Intervention & Häufigkeit \\
\hline Allgemeine klinische Beobachtung & 26 \\
Venöse Blutentnahme & 18 \\
Nicht-invasive Untersuchung & 14 \\
Erhebung psychometrischer Daten & 13 \\
Venöse Blutentnahme ohne Notwendigkeit zusätzlicher Punktion & 8 \\
Erhebung morphometrischer Daten & 7 \\
Placebogabe & 6 \\
Nicht-invasive Sammlung von Ausscheidungsprodukten & 3 \\
Sonographie & 2 \\
Transkranielle Magnetstimulation & 2 \\
Röntgenaufnahme & 2 \\
Liquorentnahme ohne Notwendigkeit zusätzlicher Punktion & 2 \\
Gewebeprobe & 2 \\
Magnetresonanztomographie & 1 \\
Intramuskuläre Injektion & 1 \\
Knochenmarkentnahme ohne Notwendigkeit zusätzlicher Punktion & 1 \\
Kontrastmitteluntersuchung & 1 \\
Elektromyogramm & 1 \\
\hline
\end{tabular}

Tabelle 8.1: Geplante Eingriffe der gruppennützigen Forschungsprojekte (Anträge an die Ethikkommission der Universität Göttingen 1999-2003, N=57) 


\subsection{Originalfragebogen}

Vignetten und Fragen in der ursprünglichen Reihenfolge, Formatierung angepasst

Prof. Dr. med. Claudia Wiesemann

Dr. med. Matthias Dahl

Katrin Radenbach

Abteilung Ethik und Geschichte der Medizin

Humboldtallee 36

D-37073 Göttingen

Tel.: 0551-394184

http://www.GWDG.DE/paracelsus/

\section{Fragebogen zur Forschung an Minderjährigen für Vorsitzende von Ethikkommissionen in Deutschland}

Der vorliegende Fragebogen enthält zunächst einen Statistikteil. Wir möchten Sie bitten, einige Angaben zu ihrer Person zu beantworten. Daran schließen sich sechs Fallvignetten an, in denen wir Sie zu konkreten Forschungsszenarien befragen möchten. Bitte teilen Sie uns mit, wie Sie über den Antrag folgender Forschungsprojekte entscheiden würden und kreuzen Sie eine der vorgegebenen Antwortmöglichkeiten an. Zusätzlich steht Ihnen Platz für weitere Anmerkungen zur Verfügung. Schließlich interessiert uns - bezogen auf die Fallvignetten - Ihre Einschätzung zu verschiedenen Aspekten der Partizipation von Kindern und Jugendlichen. Auch hierfür verwenden Sie bitte die vorgegebenen Antwortmöglichkeiten. 
1. Statistik: Zu Beginn möchten wir Sie bitten, einige Angaben zu Ihrer Person zu machen:

\subsection{Geschlecht:}

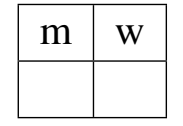

1.2 Alter:

\begin{tabular}{|l|l|l|l|l|}
\hline $20-30$ & $31-40$ & $41-50$ & $51-60$ & $>60$ \\
\hline & & & & \\
\hline
\end{tabular}

\subsection{Erlernter Beruf/Funktion in der Ethikkommission:}

\begin{tabular}{|l|l|}
\hline Pädiater/-in & \\
\hline Rechtsmediziner/-in & \\
\hline Pharmakologe/-in & \\
\hline Andere ärztliche Tätigkeit & \\
\hline Medizinische/-r Biometriker/-in und Statistiker/-in & \\
\hline Pharmazeut/-in & \\
\hline Jurist/-in & \\
\hline Theologe/-in & \\
\hline Philosoph/-in & \\
\hline Medizinethiker/-in & \\
\hline Student/-in & \\
\hline Pflegervertreter/-in & \\
\hline Bürgervertreter/-in (Laie) & \\
\hline Sonstiges & \\
\hline
\end{tabular}

1.4 An wie vielen Sitzungen einer Ethikkommission haben Sie bisher teilgenommen?

\begin{tabular}{|l|l|l|}
\hline $0-10$ & $11-50$ & $>50$ \\
\hline & & \\
\hline
\end{tabular}

\subsection{Haben Sie selbst eigene Kinder?}

\begin{tabular}{|l|l|}
\hline Ja & \\
\hline Nein & \\
\hline
\end{tabular}

1.6 Wenn ja, wie viele Kinder haben Sie und wie alt sind Ihre Kinder? 


\section{Fallvignetten}

2.1 a) Beispiel für primär nicht therapeutische Forschung, die niemals den Betroffenen selbst, aber für Patienten der gleichen Krankheits- und Altersgruppe später einen Nutzen bringen könnte.

Das Rett-Syndrom ist eine Erkrankung mit verzögerter geistiger Entwicklung, Autismus, Epilepsie und weiteren neurologischen Auffälligkeiten, das sich im Säuglingsalter manifestiert und fast ausschließlich bei Mädchen vorkommt. Als Ursache konnte eine Genmutation (MECP2) nachgewiesen werden. Aber auch bei Mädchen und Jungen mit unspezifischer verzögerter geistiger Entwicklung konnte eine Mutationen im MECP2-Gen nachgewiesen werden. Um das gesamte klinische Spektrum dieser Genmutation zu erforschen, soll das Blut von Patienten mit mentaler Retardierung unklarer Genese genetisch analysiert werden. Geplant ist eine Untersuchung von acht Gruppen mit je 15 Patienten im Alter von zwei bis fünf Jahren. Für die Untersuchung werden pro Patient fünf ml Blut benötigt, die im Rahmen ohnehin notwendiger Blutentnahmen entnommen und anonymisiert ausgewertet werden sollen.

Halten Sie eine solche Studie für vertretbar und würden Sie ein positives Votum einer Ethikkommission befürworten?

Ja, ohne Einschränkungen

$\square$ Ja, mit Einschränkungen (bitte Antwort begründen)

$\square$ Ich weiß nicht / keine Meinung

Nein, auf gar keinen Fall (bitte Antwort begründen)

Anmerkungen:

Halten Sie eine individuelle Aufklärung und Zustimmung des Kindes für erforderlich?

$\square \mathrm{Ja} \quad \square$ Nein

Wäre für Sie ein eindeutig abwehrendes Verhalten des Kindes ein Ausschluss- bzw. Abbruchkriterium?

$\square$ Ja $\quad \square$ Nein

Halten Sie eine Aufwandsentschädigung für das Kind für notwendig?

$\square$ Ja $\quad \square$ Nein 
2.1 b) Beispiel für primär nicht therapeutische Forschung, die für Kinder der gleichen Altersgruppe einen Nutzen bringt

Im Rahmen einer Studie soll das überzufallig häufige gemeinsame Auftreten von Hyperkinetischer Störung (HKS) und Lese-Rechtschreib-Schwäche (LRS) untersucht werden. Bei der beantragten Studie sollen verschiedene Ursachen beider Störungen analysiert werden. Ziel ist es, die krankheitsauslösenden Ursachen zu erklären, um eine genauere und frühzeitige Diagnostik und Therapie zu ermöglichen. Hierfür werden mindestens 80 Kinder mit Lese-Rechtschreib-Schwäche und/oder Hyperkinetischer Störung (zwischen sechs und elf Jahren) sowie zum Vergleich eine Zahl gesunder Kinder untersucht. Die Testdauer beträgt insgesamt sieben bis acht Stunden inklusive Pausen, verteilt auf drei Tage innerhalb einer Woche. Zu den Untersuchungen gehören: Neurologische Untersuchung, Hörtest, Fragebögen, etablierter Intelligenztest für das Kindesalter, Lese-RechtschreibTests, Aufmerksamkeitstests. Bei einer Sitzung wird ein Elektroenzephalogramm (EEG) abgeleitet.

Halten Sie eine solche Studie für vertretbar und würden Sie ein positives Votum einer Ethikkommission befürworten?

Ja, ohne Einschränkungen

$\square$ Ja, mit Einschränkungen (bitte Antwort begründen)

$\square$ Ich weiß nicht / keine Meinung

Nein, auf gar keinen Fall (bitte Antwort begründen)

Anmerkungen:

Halten Sie eine individuelle Aufklärung und Zustimmung des Kindes für erforderlich?

$\square$ Ja $\quad \square$ Nein

Wäre für Sie ein eindeutig abwehrendes Verhalten des Kindes ein Ausschluss- bzw. Abbruchkriterium?

$\square$ Ja $\quad \square$ Nein

Halten Sie eine Aufwandsentschädigung für das Kind für notwendig?

- Für die kranken Kinder? - Für die gesunden Kinder?

$\square$ Ja $\quad \square$ Nein $\quad \square$ Ja $\quad \square$ Nein 
2.2 a) Beispiel für therapeutische Forschung mit ausschließlich nicht therapeutischem Anteil

20 Säuglinge im Alter zwischen zwei Wochen bis drei Monaten mit angeborenen Herzfehlern und klinisch manifester Herzinsuffizienz nehmen präoperativ an einer Medikamentenstudie teil. In einer randomisierten Medikamentenstudie erhalten die Kinder der Studiengruppe (zehn Patienten) neben der Standardtherapie (Digoxin, Diuretikum) zusätzlich den Betarezeptorenblocker Propranolol, während die Kinder der Kontrollgruppe (zehn Patienten) ausschließlich mit der Standardtherapie behandelt werden. Im Rahmen der Herzoperation wird bei allen Kindern eine Herzmuskelbiopsie für rein wissenschaftliche Zwecke entnommen.

Halten Sie eine solche Studie für vertretbar und würden Sie ein positives Votum einer Ethikkommission befürworten?

$\square \mathrm{Ja}$, ohne Einschränkungen

$\square$ Ja, mit Einschränkungen (bitte Antwort begründen)

$\square$ Ich weiß nicht / keine Meinung

Nein, auf gar keinen Fall (bitte Antwort begründen)

Anmerkungen:

Halten Sie eine Aufwandsentschädigung für die Eltern für notwendig?

$\square$ Ja $\quad \square$ Nein 
2.2 b) Beispiel für therapeutische Forschung mit ausschließlich nicht therapeutischem Anteil

In einer randomisierten therapeutischen Medikamentenstudie wird bei Kindern (sechs bis elf Jahre alt) mit akuter lymphatischer Leukämie (ALL) ein neues Chemotherapieschema gegen die etablierte Therapie getestet. Im Studienverlauf werden bei beiden Gruppen im wöchentlichen Abstand Knochenmarkpunktionen vorgenommen. Die Kinder erhalten hierfür ein leichtes Schlafmittel und eine lokale Betäubung der Haut (Emla-Pflaster). Die Knochenmarkpunktionen erfolgen teilweise zur Verlaufskontrolle, teilweise zu reinen Studienzwecken. Insgesamt werden sechs von zehn Knochenmarkpunktionen ausschließlich zu wissenschaftlichen Zwecken durchgeführt. Hierbei sollen minimale molekulare Veränderungen (minimal residual disease) untersucht werden.

Halten Sie eine solche Studie für vertretbar und würden Sie ein positives Votum einer Ethikkommission befürworten?

$\square$ Ja, ohne Einschränkungen

$\square$ Ja, mit Einschränkungen (bitte Antwort begründen)

$\square$ Ich weiß nicht / keine Meinung

Nein, auf gar keinen Fall (bitte Antwort begründen)

Anmerkungen:

Halten Sie eine individuelle Aufklärung und Zustimmung des Kindes für erforderlich?

$\square$ Ja $\quad \square$ Nein

Wäre für Sie ein eindeutig abwehrendes Verhalten des Kindes ein Ausschluss- bzw. Abbruchkriterium?

$\square$ Ja $\quad \square$ Nein

Halten Sie eine Aufwandsentschädigung für das Kind für notwendig?

$\square$ Ja $\quad \square$ Nein 


\section{3 a) Beispiel für Forschung unter Einschluss eines Placeboarmes}

Im Rahmen einer randomisierten, placebokontrollierten, multizentrischen Phase-2-Doppelblindstudie erhalten Allergiker mit ganzjährig auftretendem allergischem Schnupfen (Hausstaubmilbe) ab einem Alter von zwölf Jahren einmal täglich eine Tablette eines neu entwickelten und erstmalig angewandten Antihistaminikums über einen Zeitraum von sechs Monaten. Das Medikament wird gegen Placebo getestet, da die bisherige medikamentöse Behandlung unzuverlässig ist und keine vollständige Symptomfreiheit bringt. Die allergischen Symptome werden in einem Tagebuch dokumentiert.

Halten Sie eine solche Studie für vertretbar und würden Sie ein positives Votum einer Ethikkommission befürworten?

$\square$ Ja, ohne Einschränkungen

Ja, mit Einschränkungen (bitte Antwort begründen)

$\square$ Ich weiß nicht / keine Meinung

$\square$ Nein, auf gar keinen Fall (bitte Antwort begründen)

Anmerkungen:

Halten Sie eine individuelle Aufklärung und Zustimmung des Kindes für erforderlich?

$\square$ Ja $\quad \square$ Nein

Wäre für Sie ein eindeutig abwehrendes Verhalten des Kindes ein Ausschluss- bzw. Abbruchkriterium?

$\square$ Ja $\quad \square$ Nein

Halten Sie eine Aufwandsentschädigung für das Kind für notwendig?

$\square \mathrm{Ja} \quad \square$ Nein 


\section{3 b) Beispiel für Forschung unter Einschluss eines Placeboarmes}

Kinder mit angeborenen Herzfehlern haben eine erhöhte Erkrankungshäufigkeit für Herzmuskelentzündungen, die durch RS-Viren hervorgerufen wird. Im Rahmen einer randomisierten, placebokontrollierten Studie soll getestet werden, ob eine prophylaktische Anwendung von Immunglobulinen die Erkrankungshäufigkeit für Herzmuskelentzündungen senkt. Kinder im Alter zwischen zwei und fünf Jahren und mit angeborenem Herzfehler erhalten prophylaktisch fünf Immunglobulin-Injektionen intramuskulär. Den Kindern der Kontrollgruppe werden fünf Injektionen physiologischer Kochsalzlösung intramuskulär gegeben. Im Verlauf wird beobachtet, wie viele Kinder von jeder Gruppe an Myokarditis erkranken. Die Erkrankungsfälle werden mit der Standardtherapie behandelt.

Halten Sie eine solche Studie für vertretbar und würden Sie ein positives Votum einer Ethikkommission befürworten?

Ja, ohne Einschränkungen

$\square$ Ja, mit Einschränkungen (bitte Antwort begründen)

$\square$ Ich weiß nicht / keine Meinung

Nein, auf gar keinen Fall (bitte Antwort begründen)

Anmerkungen:

Halten Sie eine individuelle Aufklärung und Zustimmung des Kindes für erforderlich?

$\square$ Ja $\quad \square$ Nein

Wäre für Sie ein eindeutig abwehrendes Verhalten des Kindes ein Ausschluss- bzw. Abbruchkriterium?

$\square$ Ja $\quad \square$ Nein

Halten Sie eine Aufwandsentschädigung für das Kind für notwendig?

$\square$ Ja $\quad \square$ Nein 


\section{Literaturverzeichnis}

Aberegg SK, Haponik EF, Terry PB (2005): Omission bias and decision making in pulmonary and critical care medicine. Chest $\underline{128}, 1497-1505$

Alderson P: Children's consent to surgery. Open University Press, Buckingham 1993

Alderson P: Die Autonomie des Kindes - über die Selbstbestimmungsfähigkeit von Kindern in der Medizin; in: Das Kind als Patient. Ethische Konflikte zwischen Kindeswohl und Kindeswille; hrsg. v. Wiesemann C, Dörries A, Wolfslast G, Simon A; Campus, Frankfurt/M. 2003, 28-47

American Academy of Pediatrics Committee on Drugs (1995): Guidelines for the ethical conduct of studies to evaluate drugs in pediatric populations. Pediatrics $\underline{95}$, 286-294

AMG (1994): Arzneimittelgesetz in der Fassung vom 9. August 1994

http: / /www.jura.uni-sb.de/BGBI/TEIL1/1994/19943019.1. HTML

AMG (2004): Arzneimittelgesetz in der Fassung vom 30. Juli 2004 http://bundesrecht.juris.de/amg_1976

Beck M, Opp KD (2001): Der faktorielle Survey und die Messung von Normen. Köln Z Sozialpsychol Soziol 53, 283-306

Beecher HK (1966): Ethics and clinical research. N Engl J Med 274, 1354-1360

Biller-Andorno N, Wild V (2003): Arzneimittelforschung an Schwangeren: Besonderer Schutz - aber kein Ausschluss aus der Forschung. Dtsch Arztebl 100, A 970-972

Blumer K: Tierversuche zum Wohle des Menschen? Ethische Aspekte des Tierversuchs unter besonderer Berücksichtigung transgener Tiere. Utz, München 1999

British Paediatric Association (1980): Guidelines to aid ethical committees considering research involving children. Working Party on Ethics of Research in Children. BMJ $\underline{280}, 229-231$

Brochhausen C: Kinder in klinischen Studien - eine interdisziplinäre Herausforderung; in: Kinder in klinischen Studien - Grenzen medizinischer Machbarkeit?; hrsg. v. Brochhausen C, Seyberth HW; Lit, Münster 2005, 17-35 
Brock DW: Ethical issues in exposing children to risks in research; in: Children as Research Subjects: Science, Ethics and Law; hrsg. v. Grodin MA, Glantz LH; Oxford University Press, Oxford 1994, 81-101

Bücheler R, Schwab M, Mörike K, Kalchthaler B, Mohr H, Schröder H, Schwoerer P, Gleiter $\mathrm{CH}$ (2002): Off label prescribing to children in primary care in Germany: retrospective cohort study. BMJ $\underline{324}, 1311-1312$

Bundesinstitut für Arzneimittel und Medizinprodukte (2005): Medizinprodukte http://www.bfarm.de/de/Medi zinprodukte/index.php

45 CFR 46 (1974): Titel 45 Code of Federal Regulations Part 46: Protection of Human Subjects

http://ohsr.od.nih.gov/guidelines/4 5cfr46.html

Conroy S, Choonara I, Impicciatore P, Mohn A, Arnell H, Rane A, Knoeppel C, Seyberth HW, Pandolfini C, Raffaelli, MP et al. (2000): Survey of unlicensed and off label drug use in paediatric wards in European countries. European Network for Drug Investigation in Children. BMJ $\underline{320}, 79-82$

Dahl M, Wiesemann C (2001): Forschung an Minderjährigen im internationalen Vergleich: Bilanz und Zukunftsperspektiven. Ethik Med 13, 87-110

Dahl M, Wiesemann C: Ethische Aspekte der Forschung mit Kindern und Jugendlichen; in: Kinder in klinischen Studien - Grenzen medizinischer Machbarkeit?; hrsg. v. Brochhausen C, Seyberth HW; Lit, Münster 2005, 75-97

Dahl M, Hoffmann J, Styllos M, Wiesemann C (2002): Ethische Aspekte neonatologischer Forschung: Die Sicht von Eltern, Pflegenden und Ärzten. Dtsch Arztebl $\underline{98}$, A 2554

Deklaration von Helsinki (2004): World Medical Association: Declaration of Helsinki. Ethical Principles for Medical Research Involving Human Subjects http://www.wma.net/e/policy/b3.htm

Deutsch E, Lippert HD: Ethikkommission und klinische Prüfung. Vom Prüfplan zum Prüfvertrag. Springer, Berlin 1998

Deutscher Ärztetag (1988): Tagesordnungspunkt "Berufsordnung". Grundgesetz für die ärztliche Tätigkeit. Dtsch Arztebl 85, A 943-948

Diekmann A: Empirische Sozialforschung. Grundlagen, Methoden, Anwendungen. 9. Auflage; Rowohlt, Reinbek 2002

Dörner K, Spielmann U: Geistige Behinderung, Humangenetik und Ethik: Der WürzburgEisinger Fall. Verlag St. Josefs-Stift, Eisingen 2001 
Eden OB (1994): Informed consent. Consent difficult in paediatric oncology. BMJ $\underline{308}$, 272

Ekins-Daukes S, Helms PJ, Simpson CR, Taylor MW, McLay JS (2004): Off-label prescribing to children in primary care: retrospective observational study. Eur J Clin Pharmacol 60, 349-353

Ethikkommission der Georg-August-Universität Göttingen (2005): Tätigkeitsberichte der Jahre 1999-2003

http://www.mi.med.uni-goettingen. de/ethik/Jahresbericht. htm

Faden RR, Beauchamp TL: The History and Theory of Informed Consent. Oxford University Press, New York 1986

Fegert JM, Wiethoff K, Dippold I, Rothärmel F, Wolfslast G: Information und Partizipation von Kindern und Jugendlichen bei Behandlungsentscheidungen in der Kinder- und Jugendpsychiatrie; in: Kinder in klinischen Studien - Grenzen medizinischer Machbarkeit?; hrsg. v. Brochhausen C, Seyberth HW; Lit, Münster 2005, 117-143

Freedman B, Fuks A, Weijer C (1993): In Loco Parentis. Minimal Risk as an Ethical Threshold for Research upon Children. Hastings Cent Rep 23 , 13-19

Friedrichs J (1974): Situation als soziologische Erhebungseinheit. Z Soz $\underline{3}$, 33-53

Fröhlich U: Forschung wider Willen? Rechtsprobleme biomedizinischer Forschung mit nichteinwilligungsfähigen Personen. Springer, Berlin 1999

Grisso T, Appelbaum PS: Assessing Competence to Consent to Treatment. A Guide for Physicians and Other Health Professionals. Oxford University Press, New York 1998

Guidelines des CIOMS (Council for International Organizations of Medical Sciences) (2002): International Ethical Guidelines for Biomedical Research Involving Human Subjects. Bull Med Ethics 182, 17-23

Gundert-Remy U: Arzneimittelrecht und Arzneimittelprüfung; in: Füllgraff Palm. Pharmakotherapie. Klinische Pharmakologie; hrsg. v. Lemmer B, Brune K; Urban und Fischer, München 2001, 1-19

Hämäläinen ML, Hoppu K, Santavuori P (1997): Sumatriptan for migraine attacks in children: a randomized placebo-controlled study. Do children with migraine respond to oral sumatriptan differently from adults. Neurology $\underline{48}, 1100-1103$

Harth SC, Thong YH (1995): Parental perceptions and attitudes about informed consent in clinical research involving children. Soc Sci Med $\underline{41}, 1647-1651$

Heintze K: Pharmaka mit Wirkung auf den Gastrointestinaltrakt; in: Pharmakologie und Toxikologie. 5. Auflage; hrsg. v. Estler CJ; Schattauer, Stuttgart 2000, 431-458 
Herold G: Innere Medizin. Eine vorlesungsorientierte Darstellung. Eigenverlag Herold, Köln 2004

Hoffmaster CB, Stewart MA, Christie RJ (1991): Ethical decision making by family doctors in Canada, Britain, and the United States. Soc Sci Med 33, 647-653

Hulpke-Wette M (2005): Arzneimitteltherapie und Arzneimittelprüfung bei Kindern und Jugendlichen. Z med Ethik 51, 182-191

Janofsky J, Starfield B (1981): Assessment of risk in research on children. J Pediatr 98, $842-846$

Just H: Die öffentlich-rechtlichen Ethikkommissionen in Deutschland - derzeitige Struktur und Arbeitsweise sowie Perspektiven der zukünftigen Entwicklung; in: Die EthikKommissionen: neuere Entwicklungen und Richtlinien; hrsg. v. Wiesing U; Deutscher Ärzteverlag, Köln 2002, 90-103

Klein J (1998): Die ethische Problematik des Tierversuchs. Ethica $\underline{6}, 383-406$

Klinkhammer G (2003): Ethikkommissionen: Verwirrende Vielfalt. Dtsch Arztebl 100, A 304-306

Kölch M: Aufklärung bei klinischen Studien mit Kindern und Jugendlichen; in: Das Kind als Patient. Ethische Konflikte zwischen Kindeswohl und Kindeswille; hrsg. v. Wiesemann C, Dörries A, Wolfslast G, Simon A; Campus, Frankfurt/M. 2003, 59-71

Kopelman LM: When is the risk minimal enough for children to be research subjects?; in: Children and Health Care: Moral and Social Issues; hrsg. v. Kopelman LM, Moskop JC; Kluwer Academic Publishers, Dordrecht 1989, 89-99

Kopelman LM (2000): Children as research subjects: a dilemma. J Med Philos 25, 745764

Laor N (1987): Toward liberal guidelines for clinical research with children. Med Law $\underline{6}$, $127-137$

Levine RJ: Children as research subjects; in: Children and Health Care: Moral and Social Issues; hrsg. v. Kopelman LM, Moskop JC; Kluwer Academic Publishers, Dordrecht 1989, 73-87

Maio G (2001a): Zur Begründung einer Ethik der Forschung an nicht einwilligungsfähigen Patienten. Z Evang Ethik $\underline{45}$, 135-148

Maio G (2001b): Zur Ethik der fremdnützigen Forschung an Kindern. Z Med Ethik 47, $173-187$

Mayring P: Einführung in die qualitative Sozialforschung. Beltz, Weinheim 2002 
McCormick RA (1976): Experimentation in children: sharing in sociality. Hastings Cent Rep 6, 41-46

McIntosh N (1993): Informed consent in clinical trials. Strengthen ethical committees' role. BMJ $\underline{307}, 1496$

McIntosh N, Bates P, Brykczynska G, Dunstan G, Goldman A, Harvey D, Larcher V, McCrae D, McKinnon A, Patton M et al. (2000): Guidelines for the ethical conduct of medical research involving children. Royal College of Paediatrics, Child Health: Ethics Advisory Committee. Arch Dis Child 2, 177-182

Menschenrechtsübereinkommen des Europarates (1997): Übereinkommen zum Schutz der Menschenrechte und der Menschenwürde im Hinblick auf die Anwendung von Biologie und Medizin: Menschenrechtsübereinkommen zur Biomedizin des Europarates

http://www.ruhr-uni-bochum.de/zme/Europarat.htm

Mitscherlich A, Mielke F (Hg.) Das Diktat der Menschenverachtung. Schneider, Heidelberg 1947

Modi N (1993): Informed consent in clinical trials. Informed consent difficult in paediatric intensive care. BMJ $\underline{307}, 1495$

MPG (1994): Medizinproduktegesetz in der Fassung vom 2. August 1994

http: / /www.jura.uni-sb.de/BGBl/TEIL1/1994/19941963.1. HTML

MPG (2002): Medizinproduktegesetz in der Fassung vom 7. August 2002 http://bundesrecht.juris.de/mpg/index.html

(Muster-)Berufsordnung (2004): (Muster-)Berufsordnung für die deutschen Ärztinnen und Ärzte

http: / / www . bundesaerztekammer.de/30/Berufsordnung/10Mbo/

Nelson RM: Children as research subjects; in: Beyond Consent: Seeking justice in research; hrsg. v. Kahn JP, Mastroianni AC, Sugarman J; Oxford University Press, New York 1998, 47-66

Neubert A, Dormann H, Weiss J, Egger T, Criegee-Rieck M, Rascher W, Brune K, Hinz B (2004): The impact of unlicensed and off-label drug use on adverse drug reactions in paediatric patients. Drug Saf 27, 1059-1067

Nicholson RH: Medical research with children: Ethics, law, and practice. Oxford University Press, Oxford 1986

Ondrusek N, Abramovitch R, Pencharz P, Koren G (1998): Empirical examination of the ability of children to consent to clinical research. J Med Ethics 24, 158-165 
Perlman NB, Freedman JL, Abramovitch R, Whyte H, Kirpalani H, Perlman M (1991): Informational needs of parents of sick neonates. Pediatrics $\underline{88}, 512-518$

Pestalozza C (2004): Risiken und Nebenwirkungen: Die Klinische Prüfung von Arzneimitteln am Menschen nach der 12. AMG-Novelle. Neue Jurist Wochenschr, NJW 47, 3374-3379

Postlethwaite RJ, Reynolds JM, Wood AJ, Evans JH, Lewis MA, Eminson DM (1995): Recruiting patients to clinical trials: lessons from studies of growth hormone treatment in renal failure. Arch Dis Child 73, 30-35

Ramsey P (1976): The enforcement of morals: nontherapeutic research on children. Hastings Cent Rep $\underline{6}, 21-30$

Ramsey P (1977a): Children as research subjects: a reply. Hastings Cent Rep $\underline{7}, 40-42$

Ramsey P (1977b): Ordinary risks of childhood. Hastings Cent Rep ㄱ, 4

Raspe H (2005): Klinische Studien - Beratungsleitfaden bereitgestellt. Dtsch Ärztebl 102, A 3166

Raspe H, Hüppe A, Steinmann M: Empfehlungen zur Begutachtung klinischer Studien durch Ethik-Kommissionen. Deutscher Ärzte-Verlag, Köln 2005

Rassner G: Dermatologie: Lehrbuch und Atlas. Urban und Fischer, München 2002

Resch K, Szamel M: Therapie mit Immunsuppressiva und Immunmodulatoren; in: Füllgraff Palm. Pharmakotherapie. Klinische Pharmakologie; hrsg. v. Lemmer B, Brune K; Urban und Fischer, München 2001, 173-182

Richtlinie 2001/20/EG (2001): Richtlinie 2001/20/EG des Europäisches Parlament und Rates vom 4. April 2001 zur Angleichung der Rechts- und Verwaltungsvorschriften der Mitgliedstaaten über die Anwendung der guten klinischen Praxis bei der Durchführung von klinischen Prüfungen mit Humanarzneimitteln

http://europa.eu.int/eur-lex/pri/de/oj/dat/2001/1_121/1_ 12120010501 de00340044.pdf

Roberts LW, Warner TD, Hammond KAG, Brody JL, Kaminsky A, Roberts BB (2005): Teaching medical students to discern ethical problems in human clinical research studies. Acad Med 80, 925-930

Röntgenverordnung (2003): Verordnung über den Schutz vor Schäden durch Röntgenstrahlen in der Fassung vom 30. April 2003

http://bundesrecht.juris.de/r_v_1987/index.html\#

BJNR001140987BJNE001402377 
Rosenthal W, Seyberth HW: Besonderheiten der Arzneimitteltherapie im Kindesalter; in: Füllgraff Palm. Pharmakotherapie. Klinische Pharmakologie; hrsg. v. Lemmer B, Brune K; Urban und Fischer, München 2001, 438-446

Rossi PH: Vignette analysis: Uncovering the normative structure of complex judgements; in: Qualitative and quantitative social research: Papers in honor of Paul F. Lazarsfeld; hrsg. v. Merton RK, Coleman JS, Rossi PH; Free Press, New York 1979, 176-186

Schirm E, Tobi H, de Jong-van den Berg LTW (2002): Unlicensed and off label drug use by children in the community: cross sectional study. BMJ 324, 1312-1313

Scholz H: Virale Krankheiten; in: Pädiatrie. 2. Auflage; hrsg. v. Sitzmann FC; Georg Thieme, Stuttgart 2002, 583-617

Schöne-Seifert B, Eickhoff C (1996): Behandlungsverzicht bei Schwerstkranken: Wie würden Ärzte und Pflegekräfte entscheiden? Eine Vignetten-Studie. Ethik Med $\underline{8}$, $183-216$

Schroeder-Kurth TM: Die Krankheitsursachenforschung bei der Fanconi-Anämie - ein Beispiel für die nicht-therapeutische Forschung mit Kindern; in: Das Kind als Patient. Ethische Konflikte zwischen Kindeswohl und Kindeswille; hrsg. v. Wiesemann C, Dörries A, Wolfslast G, Simon A; Campus, Frankfurt/M. 2003, 281-301

Seyberth HW (1999): Memorandum der Deutschen Gesellschaft für Kinderheilkunde und Jugendmedizin zu der arzneimittelrechtlich nicht zugelassenen Arzneimittelanwendung in der Pädiatrie. Monatsschr Kinderheilkd 147, 155

Seyberth HW: Pharmakologische Besonderheiten im Kindes- und Jugendalter; in: Kinder in klinischen Studien - Grenzen medizinischer Machbarkeit?; hrsg. v. Brochhausen C, Seyberth HW; Lit, Münster 2005, 37-50

Shirkey H (1968): Therapeutic orphans. J Pediatr $\underline{72}$, 119-120

Snowdon C, Garcia J, Elbourne D (1998): Reactions of participants to the results of a randomised controlled trial: exploratory study. BMJ $\underline{317}, 21-26$

Stellungnahme der ZEKO (1997): Stellungnahme der „Zentralen Ethikkommission“ bei der Bundesärztekammer „Zum Schutz nicht-einwilligungsfähiger Personen in der medizinischen Forschung“. Dtsch Arztebl 94, 1011-1012

Stellungnahme der ZEKO (2004): Stellungnahme der Zentralen Kommission zur Wahrung ethischer Grundsätze in der Medizin und ihren Grenzgebieten (Zentrale Ethikkommission) bei der Bundesärztekammer zur Forschung mit Minderjährigen vom 28. 04. 2004. Dtsch Arztebl 101, A 1613-1617 
Strahlenschutzverordnung (2001): Strahlenschutzverordnung in der Fassung vom 20. Juli 2001

http: //bundesrecht.juris.de/strlschv_2001/BJNR171410001. html\#BJNR171410001BJNG000200000

Taupitz J: Internationale Regeln zur medizinischen Forschung an Minderjährigen; in: Atypische Neuroleptika in der Jugendpsychiatrie; hrsg. v. Fegert JM, Häßler F, Rothärmel S; Woikowski 1999, 47-68

Taupitz J (2001): Die neue Deklaration von Helsinki. Vergleich mit der bisherigen Fassung. Dtsch Arztebl 98, A 2413-2420

Tobias JS, Souhami RL (1993): Fully informed consent can be needlessly cruel. BMJ $\underline{307}$, 1199-1201

Turner S, Nunn AJ, Fielding K, Choonara I (1999): Adverse drug reactions to unlicensed and off-label drugs on paediatric wards: a prospective study. Acta Paediatr $\underline{88}, 965-$ 968

Ufer M, Kimland E, Bergman U (2004): Adverse drug reactions and off-label prescribing for paediatric outpatients: a one-year survey of spontaneous reports in Sweden. Pharmacoepidemiol Drug Saf $\underline{13}, 147-152$

van Stuijvenberg M, Suur MH, de Vos S, Tjiang GC, Steyerberg EW, Derksen-Lubsen G, Moll HA (1998): Informed consent, parental awareness, and reasons for participating in a randomised controlled study. Arch Dis Child $\underline{79}, 120-125$

Vollmann J (2000): ,Therapeutische“ versus ,nicht-therapeutische“ Forschung - eine medizinethisch plausible Differenzierung. Ethik Med $\underline{12}, 65-74$

Wachenhausen H (2000): Möglichkeiten und Grenzen der biomedizinischen Forschung an Einwilligungsunfähigen. Recht Polit Gesundheitswes $\underline{6}, 81-90$

Walter-Sack I, Haefeli WE (2001): Klinische Studien: Arzneimittelsicherheit auch für Kinder. Dtsch Arztebl 98, A 447-449

Weithorn LA, Campbell SB (1982): The competency of children and adolescents to make informed treatment decisions. Child Dev 53, 1589-1598

Wemhöner G, Frehse M (2004): Haftungsrechtliche Aspekte bei der Ärztlichen Arzneimittelverordnung und Arzneimittelanwendung. Neue Jurist Wochenschr, NJW 129, $327-329$

Wertz DC, Fletcher JC (1989): Ethics and genetics: an international survey. Hastings Cent Rep Spec Suppl, 20-24

Wiesemann C (2005): Ethische Probleme und rechtliche Regelung der Forschung an Kindern und Jugendlichen. Z Med Ethik 51, 129-138 
Wiesemann C, Biller-Andorno N: Medizinethik. Für die neue AO. Thieme, Stuttgart 2005

Wiesemann C, Dahl M: Forschung mit Kindern und Jugendlichen: Ist eine neue gesetzliche Regelung notwendig?; in: Das Kind als Patient. Ethische Konflikte zwischen Kindeswohl und Kindeswille; hrsg. v. Wiesemann C, Dörries A, Wolfslast G, Simon A; Campus, Frankfurt/M. 2003, 264-280

Yaffe SJ (1983): Problems of drug testing in children in the United States. Pediatr Pharmacol (New York) 3 , 339-348

ZEKO (2005): Statut der Zentralen Kommission zur Wahrung ethischer Grundsätze in der Medizin und ihren Grenzgebieten (Zentrale Ethikkommission) in der am 14. 10. 1994 verabschiedeten Fassung

http://www.zentrale-ethikkommission.de/20/10statut.html

Ein Teil der Ergebnisse der vorliegenden Arbeit fand Eingang in folgende Publikation:

Lenk C*, Radenbach K*, Dahl M, Wiesemann C (2004): Non-therapeutic research with minors: how do chairpersons of German research ethics committees decide? J Med Ethics $30,85-87 ; *$ Both authors contributed equally to this article 


\section{Danksagung}

Ich danke allen, mit deren Unterstützung ich diese Arbeit verwirklichen konnte.

Besonders bedanke ich mich bei Frau Prof. Dr. Claudia Wiesemann für die geduldige und freundliche Betreuung der Arbeit, für viele anregende, bereichernde Diskussionen und für ihre konstruktive Kritik. Ihr Interesse an dem Projekt und an der Fertigstellung der Arbeit haben entscheidend dazu beigetragen, dass ich immer mit viel Freude und Begeisterung an dem Thema gearbeitet habe.

Frau Dr. Meike Quaas hat mich bei der Auswertung der Anträge an die Ethikkommission der Georg-August-Universität Göttingen unterstützt. Dafür bedanke ich mich herzlich.

Weiterhin gilt mein Dank Dr. Matthias Dahl für die Betreuung zu Beginn des empirischen Projektes und für Korrekturen. Dank auch an Anna-Karina Jacovljevic und Dr. Christian Lenk für Anregungen und an alle Mitarbeiter der Abteilung für Ethik und Geschichte der Medizin der Universität Göttingen für die freundliche Atmosphäre. Von gegenseitiger Motivation und von inhaltlichem Austausch mit meinen Mitdoktorandinnen Marianthi Styllos und Verina Wild habe ich sehr profitiert.

Ganz besonderer Dank geht an Dr. Wolfgang Barthel für Hilfe zu jeder Zeit und für seine Geduld in den letzten Wochen, außerdem an Karsten Roeseler sowie an Dr. Jörg Hahn, Yvonne Jäger, Niklas Radenbach, Jens Werner und an meine Eltern Waltraut und Dr. Detlef Radenbach für ihre Unterstützung, für Korrekturen und Anmerkungen.

Mein spezieller Dank richtet sich an die Vorsitzenden der deutschen Ethikkommissionen, die sich an der Vignettenstudie beteiligt haben.

Diese Untersuchung wurde finanziell durch die VolkswagenStiftung unterstützt. 


\section{Lebenslauf}

Am 02.08. 1978 wurde ich als erstes Kind der Sozialwissenschaftlerin Waltraut Radenbach, geb. Becher, und des Pneumologen und Sozialmediziners Dr. Detlef Radenbach in Lübeck geboren. Ich habe drei jüngere Geschwister.

Nach dem Abitur an der Freien Waldorfschule Lübeck im Sommer 1998 und einem fünfmonatigen Freiwilligendienst in einer Einrichtung für lernbehinderte Erwachsene in Kilkenny, Irland, begann ich zum Sommersemester 1999 das Medizinstudium an der Georg-August-Universität in Göttingen. Die ärztliche Vorprüfung absolvierte ich im März 2001.

Im Anschluss an den ersten Abschnitt der ärztlichen Prüfung im März 2002 studierte ich für sieben Monate in Frankreich an der Université Lille 2. Dem zweiten Abschnitt der ärztlichen Prüfung im September 2004 folgte das Praktische Jahr, das ich an Universitätskliniken in Paris, Göttingen und Montréal in den Fächern Allgemeinchirurgie, Psychiatrie und Psychotherapie, Pneumologie und Hämatologie/Onkologie absolvierte. Im Dezember 2005 erhielt ich nach dem dritten Abschnitt der ärztlichen Prüfung die ärztliche Approbation. Seit März 2006 bin ich als Assistenzärztin an der Klinik für Psychiatrie und Psychotherapie des Universitätsklinikums Aachen tätig.

Seit Sommer 2001 promoviere ich an der Abteilung für Ethik und Geschichte der Medizin in Göttingen unter der Anleitung von Frau Prof. Dr. Claudia Wiesemann. 